\title{
DFT Study on Rhodium-Catalyzed Intermolecular [2+2] \\ Cycloaddition of Terminal Alkynes with Electron-Deficient Alkenes
}

\author{
Liu Zhao, ${ }^{\text {a Lei Zhanga and De-Cai Fanga* }}$ \\ ${ }^{a}$ College of Chemistry, Beijing Normal University, Beijing 100875, China
}

Email: dcfang@bnu.edu.cn

Table S1-1. Frequencies of some stationary points for the ligand exchange process, computed at IDSCRF (PCM)-B3LYP/DZVP level in DMA.

Table S1-2. Frequencies of some stationary points for the entire catalytic cycle, computed at IDSCRF (PCM)-B3LYP/DZVP level in DMA.

Table S1-3. Frequencies of some stationary points for the alternative pathways, computed at IDSCRF (PCM)-B3LYP/DZVP level in DMA.

Table S1-4. Frequencies of some stationary points for the rate determining step when phosphine ligand is $\mathrm{P}\left(4-\mathrm{F}_{3} \mathrm{CC}_{6} \mathrm{H}_{4}\right)_{3}, \mathrm{PPh}_{2}\left(\mathrm{C}_{6} \mathrm{~F}_{5}\right)$ or $\mathrm{P}(\mathrm{OMe})_{3}$, computed at IDSCRF (PCM)-B3LYP/DZVP level in DMA.

Table S1-5. Frequencies of some stationary points for the ligand exchange steps with the introduction of $\mathrm{CsCl}$, LiF, NaF or KF, computed at IDSCRF (PCM)-B3LYP/DZVP level in DMA.

Table S1-6. Frequencies of some stationary points for the entire catalytic cycle when $\mathrm{Q}=2$-methyl-8-quinolinolato, computed at IDSCRF (PCM)-B3LYP/DZVP level in DMA.

Table S2-1. The electronic energies (Eele/a.u.), total energies ( $E_{\text {tot }} /$ a.u. $\left.=E_{\text {ele }} / a . u .+Z P E / a . u.\right)$, enthalpy (H/a.u.), Gibbs free energies in gas ( $\mathrm{G}_{1} /$ a.u. $)$ and Gibbs free energies in solution $\left(\mathrm{G}_{2} /\right.$ a.u. $)$ for the ligand exchange process, computed at IDSCRF(PCM)-B3LYP/DZVP level in DMA at $353 \mathrm{~K}$.

Table S2-2. The electronic energies ( $E_{\text {ele }} / \mathrm{a}$.u. $)$, total energies $\left(\mathrm{E}_{\mathrm{tot}} / \mathrm{a} \cdot \mathrm{u} .=\mathrm{E}_{\mathrm{ele}} / \mathrm{a}\right.$.u. $+\mathrm{ZPE} / \mathrm{a}$.u. $)$, enthalpy (H/a.u.), Gibbs free energies in gas $\left(\mathrm{G}_{1} / \mathrm{a}\right.$.u.) and Gibbs free energies in solution $\left(\mathrm{G}_{2} /\right.$ a.u. $)$ for the entire catalytic cycle, computed at IDSCRF(PCM)-B3LYP/DZVP level in DMA at 353K.

Table S2-3. The electronic energies ( $E_{\text {ele }} / \mathrm{a}$.u. $)$, total energies $\left(E_{\text {tot }} /\right.$ a.u. $=E_{\text {ele }} /$ a.u. $\left.+Z P E / a . u.\right)$, enthalpy (H/a.u.), Gibbs free energies in gas $\left(\mathrm{G}_{1} / \mathrm{a}\right.$. u. $)$ and Gibbs free energies in solution $\left(\mathrm{G}_{2} /\right.$ a.u. $)$ for the alternative pathways, computed at IDSCRF(PCM)-B3LYP/DZVP level in DMA at 353K.

Table S2-4. The electronic energies ( $E_{\text {ele }} / \mathrm{a}$.u. $)$, total energies $\left(E_{\text {tot }} /\right.$ a.u. $=E_{\text {ele }} /$ a.u. $\left.+Z P E / a . u.\right)$, enthalpy (H/a.u.), Gibbs free energies in gas $\left(\mathrm{G}_{1} / \mathrm{a}\right.$. u. $)$ and Gibbs free energies in solution $\left(\mathrm{G}_{2} /\right.$ a.u. $)$ 
for the rate determining step when phosphine ligand is $\mathrm{P}\left(4-\mathrm{F}_{3} \mathrm{CC}_{6} \mathrm{H}_{4}\right)_{3}, \mathrm{PPh}_{2}\left(\mathrm{C}_{6} \mathrm{~F}_{5}\right)$ or $\mathrm{P}(\mathrm{OMe})_{3}$, computed at IDSCRF(PCM)-B3LYP/DZVP level in DMA at 353K.

Table S2-5. The electronic energies (E $E_{\text {ele }} /$ a.u. $)$, total energies $\left(E_{\text {tot }} /\right.$ a.u. $=E_{\text {ele }} /$ a.u. $\left.+Z P E / a . u.\right)$, enthalpy (H/a.u.), Gibbs free energies in gas $\left(\mathrm{G}_{1} /\right.$ a.u. $)$ and Gibbs free energies in solution $\left(\mathrm{G}_{2} /\right.$ a.u. $)$ for the ligand exchange steps with the introduction of $\mathrm{CsCl}, \mathrm{LiF}, \mathrm{NaF}$ or $\mathrm{KF}$, computed at IDSCRF(PCM)-B3LYP/DZVP level in DMA at 353K.

Table S2-6. The electronic energies ( $E_{\text {ele }} /$ a.u. $)$, total energies $\left(E_{\text {tot }} /\right.$ a.u. $=E_{\text {ele }} /$ a.u. $\left.+Z P E / a . u.\right)$, enthalpy (H/a.u.), Gibbs free energies in gas $\left(\mathrm{G}_{1} /\right.$ a.u. $)$ and Gibbs free energies in solution $\left(\mathrm{G}_{2} /\right.$ a.u. $)$ for the entire catalytic cycle when $\mathrm{Q}=2$-methyl-8-quinolinolato, computed at IDSCRF(PCM)-B3LYP/DZVP level in DMA at 353K. (simplified model: $\mathrm{PPh}_{3}$ as ligand)

Table S2-7. The electronic energies (Eele $/$ a.u.) for the fragments of INT2/INT2b/INT2c/INT2d and TS4/TS4b/TS4c/TS4d, computed at IDSCRF(PCM)-B3LYP/DZVP level in DMA at 353K.

Table S2-8. The electronic energies (E ele $_{\text {a.u. }}$ ) for CAT, INT1, INT2, INT3, INT4 and INT5 with different spin multiplicities.

Figure S1. Gibbs free energy profiles $\left(\mathrm{kcal} \cdot \mathrm{mol}^{-1}\right)$ for the optimal ligand exchange process, computed at B3LYP+IDSCRF/DZVP level in acetonitrile at 353K.

Figure S2. Gibbs free energy profiles for the ligand and substrate exchange process with the presence of 2-methyl-8-quinolinolato, along with geometrical structures of preCAT, preCATm, CAT, CATm and some transition states, computed at B3LYP+IDSCRF/DZVP level in acetonitrile at $353 \mathrm{~K}$. (simplified model: $\mathrm{PPh}_{3}$ as ligand)

Figure S3. Gibbs free energy profiles $\left(\mathrm{kcal} \cdot \mathrm{mol}^{-1}\right)$ for the entire catalytic cycle when $\mathrm{Q}=2$-methyl-8-quinolinolato, computed at B3LYP+IDSCRF/DZVP level in acetonitrile at 353K. (simplified model: $\mathrm{PPh}_{3}$ as ligand)

Figure S4. Gibbs free energy profiles $\left(\mathrm{kcal} \cdot \mathrm{mol}^{-1}\right)$ for the ligand exchange and subsequent steps when $\mathrm{KF}, \mathrm{NaF}$ and $\mathrm{LiF}$ as base, computed at B3LYP+IDSCRF/DZVP level in acetonitrile at 353K.

Figure S5. Gibbs free energy profiles $\left(\mathrm{kcal} \cdot \mathrm{mol}^{-1}\right)$ for the ligand exchange process with the introduction of $\mathrm{CsF}$ and $\mathrm{CsCl}$, along with geometrical structures of stationary points along the reaction pathway, computed at B3LYP+IDSCRF/DZVP level in acetonitrile at 353K. 
Table S1-1. Frequencies of some stationary points for the ligand exchange process, computed at IDSCRF (PCM)-B3LYP/DZVP level in DMA.

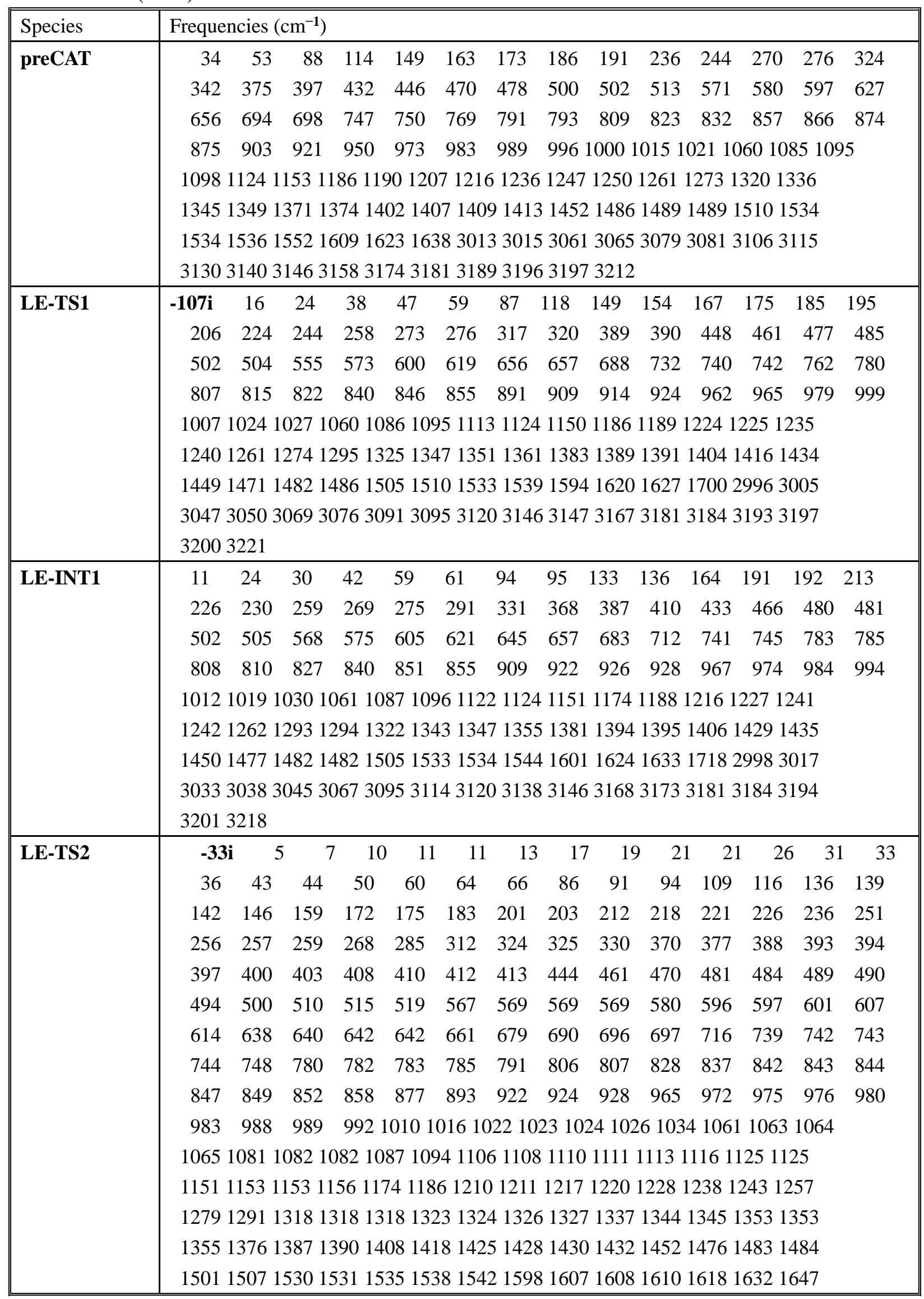




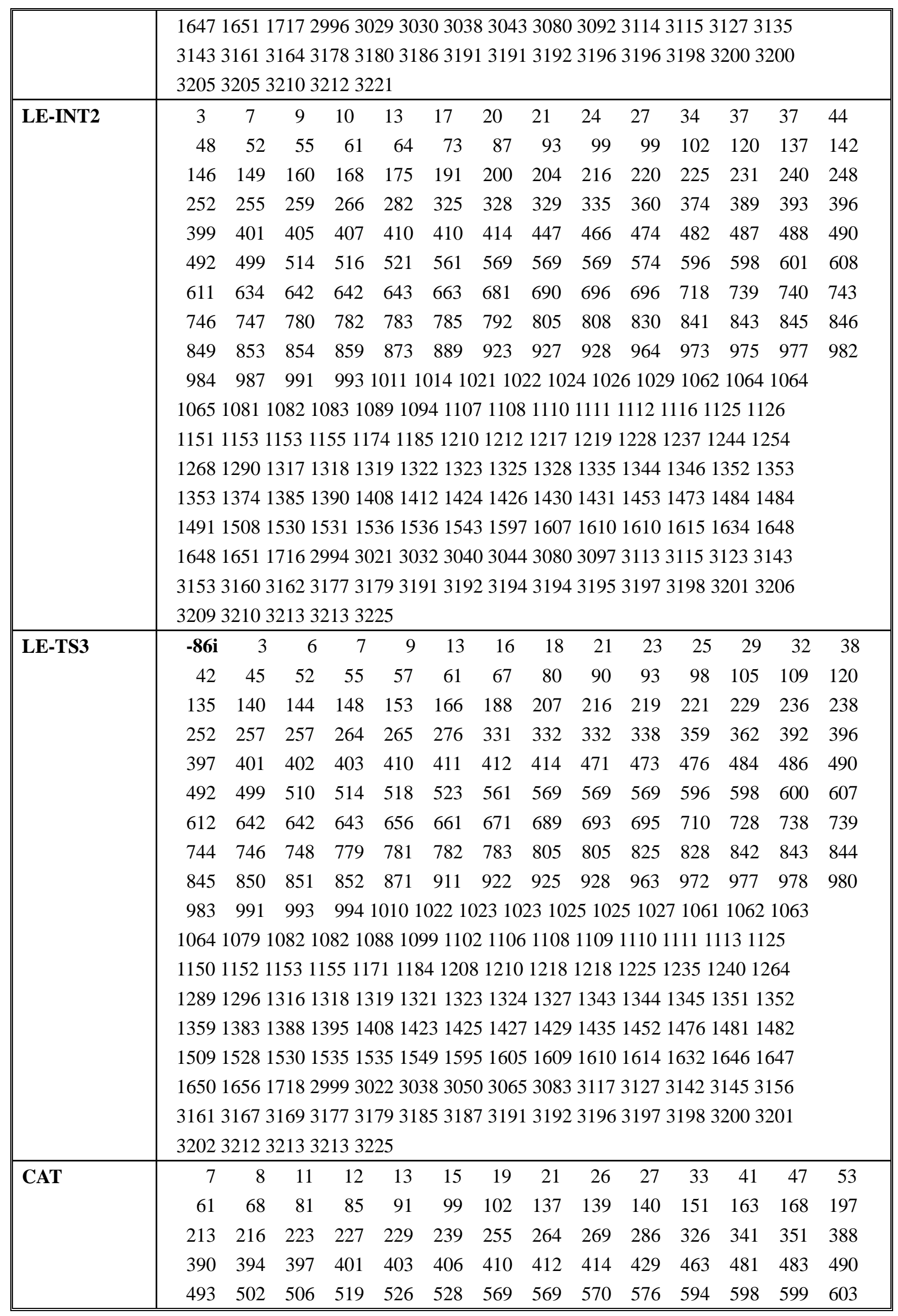

s4 


\begin{tabular}{|c|c|}
\hline & $\begin{array}{rrrrrrrrrrrrrr}621 & 641 & 642 & 643 & 656 & 691 & 696 & 696 & 740 & 743 & 744 & 744 & 747 & 781 \\
782 & 783 & 783 & 808 & 826 & 828 & 844 & 845 & 848 & 852 & 852 & 856 & 922 & 925 \\
965 & 966 & 976 & 980 & 983 & 983 & 992 & 996 & 1024 & 1024 & 1026 & 1062 & 1064 & 1065\end{array}$ \\
\hline $\mathbf{P P h}_{3}$ & 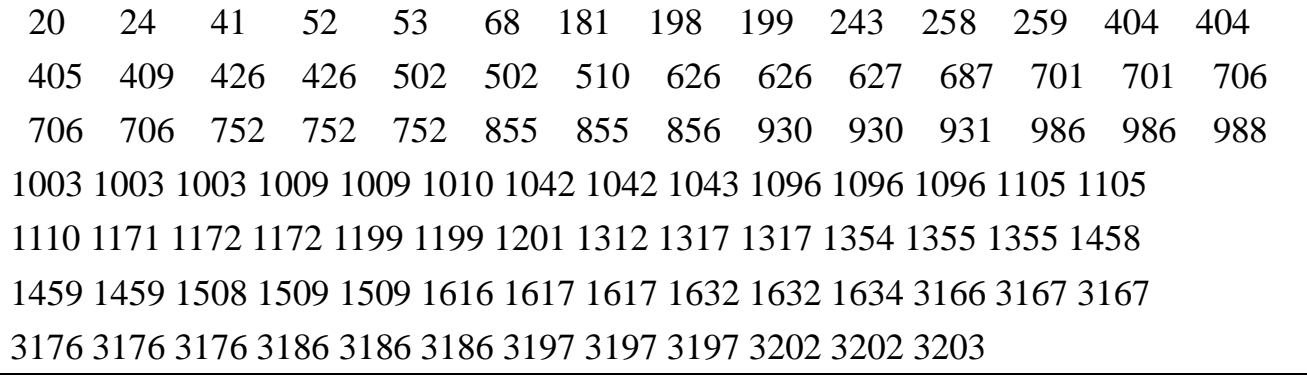 \\
\hline $\operatorname{cod}$ & 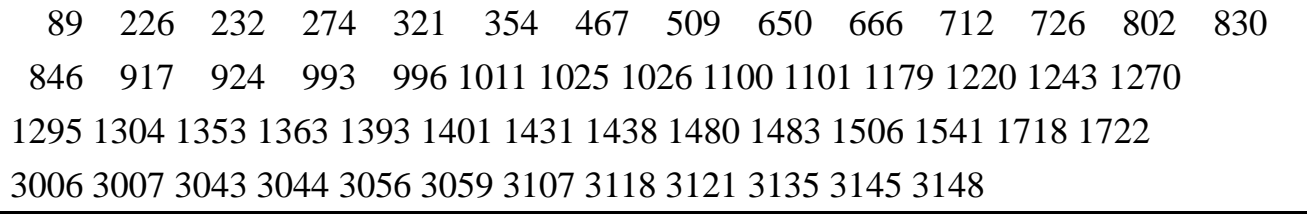 \\
\hline CsF & 174 \\
\hline LE-TS1a & 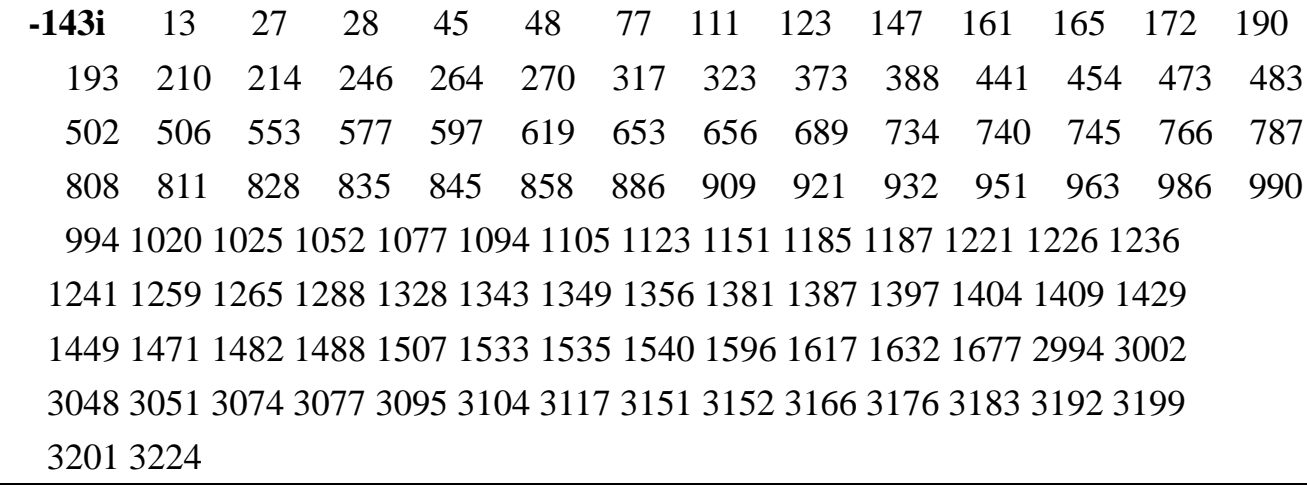 \\
\hline LE-TS1b & 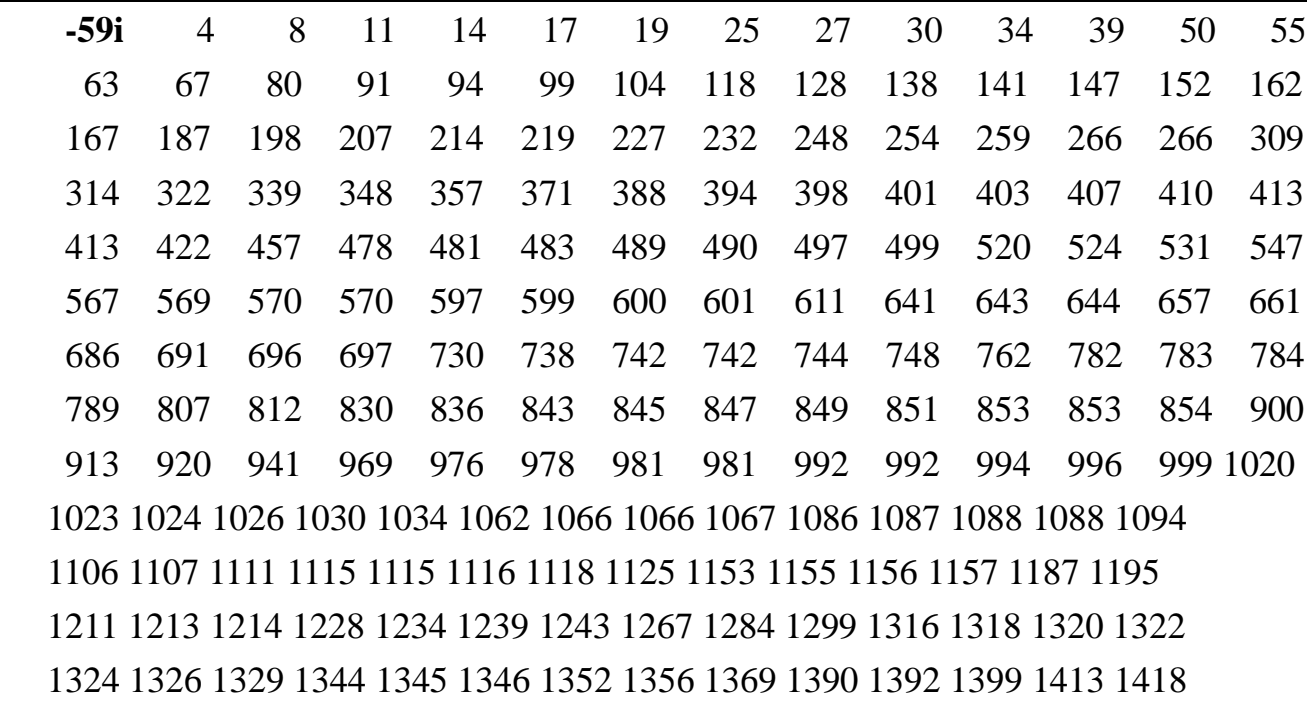 \\
\hline
\end{tabular}




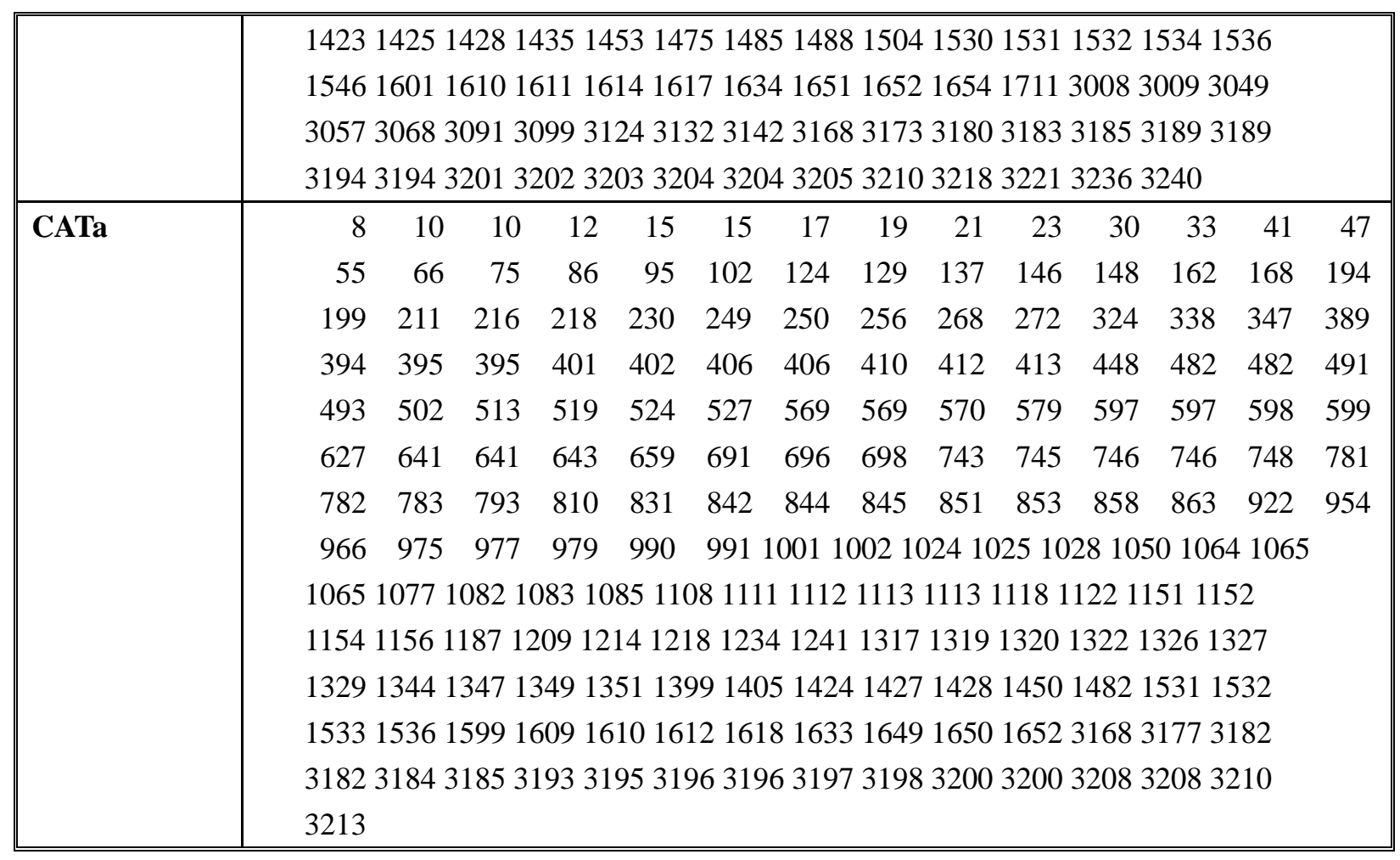

Table S1-2. Frequencies of some stationary points for the entire catalytic cycle, computed at IDSCRF (PCM)-B3LYP/DZVP level in DMA.

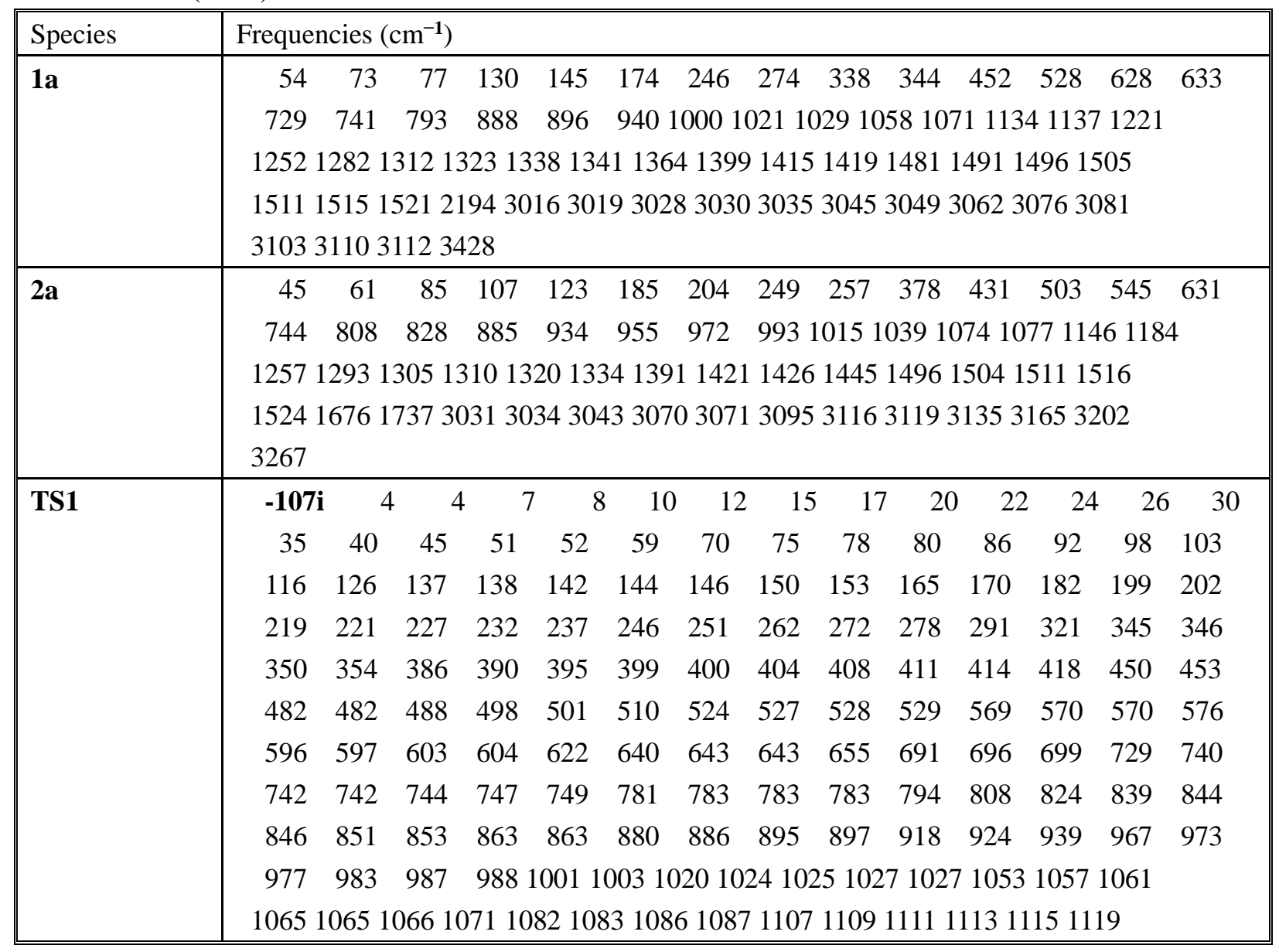




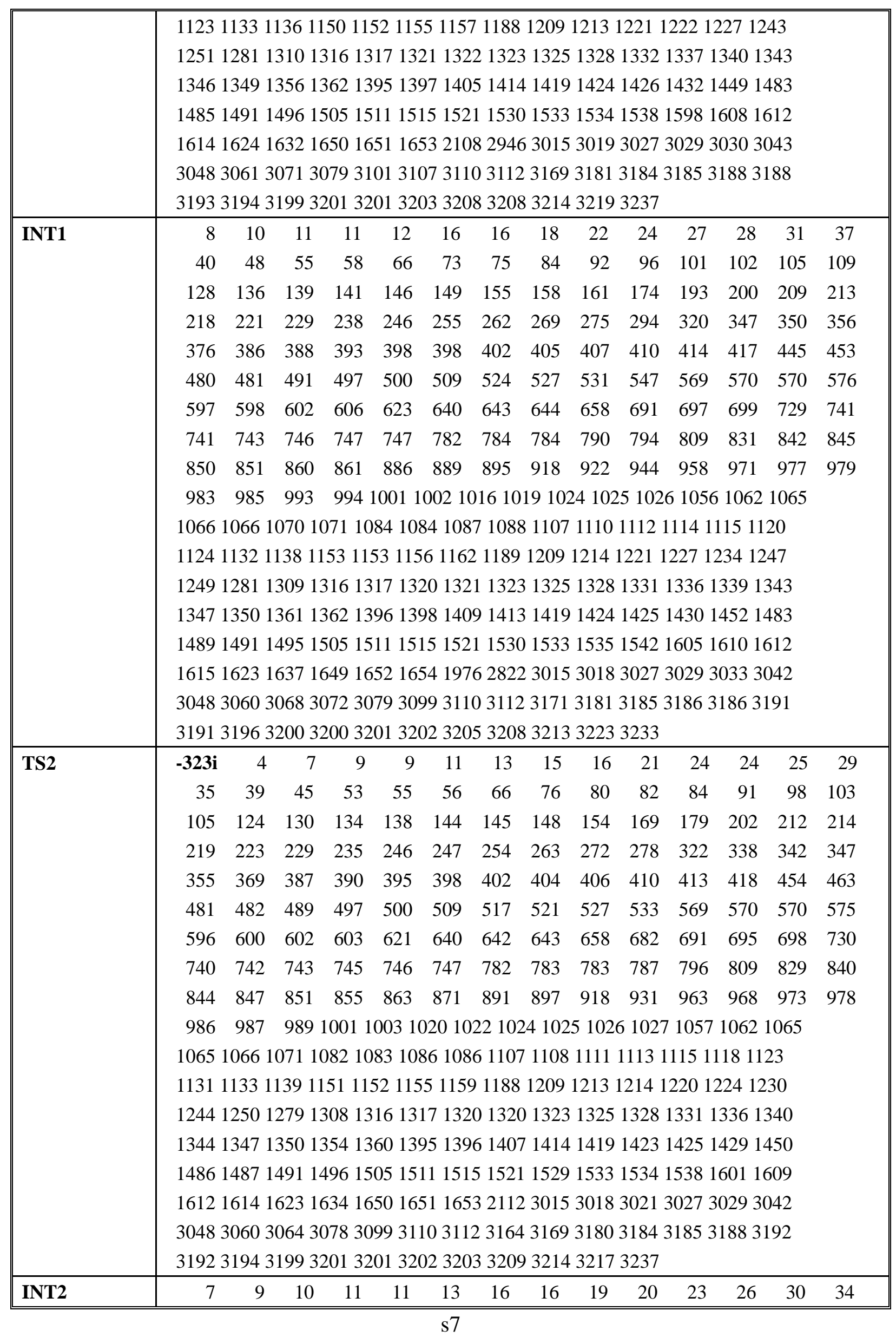




\begin{tabular}{|c|c|c|c|c|c|c|c|c|c|c|c|c|c|c|}
\hline & 39 & 40 & 47 & 51 & 58 & 62 & 65 & 78 & 79 & 84 & 94 & 97 & 101 & 106 \\
\hline & 117 & 125 & 134 & 135 & 140 & 143 & 149 & 154 & 166 & 170 & 182 & 201 & 210 & 213 \\
\hline & 226 & 228 & 236 & 246 & 247 & 257 & 264 & 271 & 290 & 308 & 319 & 345 & 358 & 369 \\
\hline & 375 & 387 & 390 & 395 & 400 & 401 & 404 & 405 & 409 & 411 & 415 & 419 & 456 & 459 \\
\hline & 481 & 482 & 487 & 496 & 499 & 505 & 520 & 524 & 531 & 538 & 569 & 570 & 570 & 574 \\
\hline & 596 & 600 & 602 & 602 & 618 & 641 & 643 & 644 & 662 & 690 & 694 & 696 & 729 & 737 \\
\hline & 740 & 743 & 745 & 746 & 749 & 781 & 782 & 782 & 788 & 792 & 804 & 810 & 829 & 833 \\
\hline & 842 & 844 & 848 & 852 & 853 & 857 & 867 & 888 & 896 & 915 & 943 & 961 & 968 & 974 \\
\hline & 976 & 985 & 990 & 991 & 993 & 10001 & 00210 & 02310 & 2410 & 24102 & 6103 & 11058 & 1061 & \\
\hline & 1064 & 10641 & 06610 & 07110 & 84108 & 84108 & 4108 & 61105 & 1108 & 1110 & 1121 & 11311 & & \\
\hline & 1123 & 1321 & 13411 & 5111 & 51115 & 4115 & 61188 & 1209 & 1212 & 12141 & 22112 & 23212 & & \\
\hline & 1248 & 12811 & 30913 & 31513 & 19132 & 20132 & 2132 & 21323 & 1325 & 1329 & 13351 & 3401 & 343 & \\
\hline & 1345 & 13461 & 35213 & 36213 & 96139 & 96141 & 11413 & 31419 & 1422 & 1424 & 14281 & 4511 & & \\
\hline & 1487 & 14911 & 49515 & 50515 & 11151 & 15152 & 11530 & 01531 & 1532 & 1535 & 6011 & 60910 & 611 & \\
\hline & 1613 & 16231 & 63716 & 54916 & 50165 & 52213 & 5292 & 93012 & 3015 & 3016 & 30263 & 0293 & 039 & \\
\hline & 3045 & 30523 & 05930 & 07630 & 95310 & 09311 & 13160 & 63179 & 3180 & 3181 & 31823 & 1873 & & \\
\hline & 3199 & 32003 & 20032 & 20132 & 02320 & 02320 & 3321 & 63220 & 3237 & 3259 & & & & \\
\hline \multirow[t]{23}{*}{ TS3 } & $-319 i$ & 5 & 6 & 8 & 9 & 10 & 11 & 14 & 15 & 16 & 17 & 21 & 23 & 25 \\
\hline & 28 & 29 & 33 & 35 & 36 & 40 & 40 & 43 & 48 & 51 & 57 & 63 & 68 & 73 \\
\hline & 74 & 77 & 79 & 85 & 91 & 98 & 99 & 104 & 108 & 120 & 124 & 134 & 135 & 140 \\
\hline & 141 & 147 & 152 & 153 & 157 & 170 & 189 & 194 & 209 & 212 & 219 & 221 & 226 & 233 \\
\hline & 240 & 246 & 248 & 249 & 258 & 260 & 265 & 269 & 273 & 285 & 320 & 343 & 355 & 372 \\
\hline & 386 & 389 & 394 & 397 & 399 & 401 & 404 & 404 & 406 & 409 & 412 & 413 & 432 & 432 \\
\hline & 454 & 462 & 480 & 481 & 490 & 494 & 497 & 502 & 511 & 520 & 525 & 533 & 555 & 569 \\
\hline & 570 & 570 & 572 & 578 & 597 & 600 & 600 & 601 & 625 & 633 & 641 & 642 & 643 & 659 \\
\hline & 674 & 681 & 692 & 697 & 698 & 728 & 740 & 742 & 743 & 744 & 744 & 748 & 748 & 767 \\
\hline & 782 & 783 & 784 & 792 & 794 & 810 & 810 & 814 & 832 & 841 & 845 & 847 & 851 & 853 \\
\hline & 857 & 859 & 862 & 887 & 895 & 902 & 918 & 929 & 949 & 955 & 964 & 971 & 974 & 979 \\
\hline & 984 & 990 & 994 & 998 & 999 & 999 & 10231 & 0231 & 2410 & 2610 & 28103 & 7106 & 01063 & \\
\hline & 1066 & 10671 & 06710 & 6810 & $7110^{7}$ & 75108 & 5108 & 81089 & 1089 & 1109 & 1101 & 11111 & & \\
\hline & 1115 & 1161 & 1711 & 24113 & 36113 & 81143 & 1153 & 1155 & 11571 & 1581 & 6911 & 84119 & & \\
\hline & 1211 & 12151 & 21712 & 2212 & 26123 & 35124 & 9125 & 11253 & 1273 & 1283 & 2981 & 3131 & 313 & \\
\hline & 1317 & 13201 & 32013 & 32113 & 22132 & 24132 & 5133 & 01331 & 1335 & 1339 & 13441 & 3471 & 348 & \\
\hline & 1350 & 13651 & 38413 & 39814 & 00140 & 08141 & 1141 ? & 31418 & 1420 & 1423 & 14251 & 4281 & 437 & \\
\hline & 1452 & 14821 & 48914 & 49114 & 9614 & 96150 & 2150 & 51505 & 1511 & 1511 & 5151 & 51815 & 521 & \\
\hline & 1528 & 15311 & 53315 & 53415 & 36160 & 05160 & 9161 & 11612 & 1615 & 1624 & 6381 & 6511 & 652 & \\
\hline & 1654 & 19182 & 81430 & 1230 & 1430 & 19302 & 6302 & 73029 & 3031 & 3036 & 30363 & 0443 & & \\
\hline & 3057 & 30593 & 06330 & 07630 & 8130 & 96310 & 4310 & 93111 & 3113 & 31153 & 1353 & 17131 & & \\
\hline & 3181 & 31853 & 19031 & 19231 & 93319 & 96319 & 7320 & 13202 & 3204 & 3205 & 32083 & 2103 & 213 & \\
\hline & 3217 & 32193 & 22832 & & & & & & & & & & & \\
\hline \multirow[t]{4}{*}{ INT3 } & 6 & 7 & 8 & 11 & 12 & 12 & 15 & 16 & 17 & 18 & 19 & 23 & 25 & 27 \\
\hline & 29 & 30 & 34 & 36 & 39 & 42 & 44 & 49 & 54 & 55 & 60 & 63 & 68 & 73 \\
\hline & 74 & 79 & 86 & 90 & 99 & 103 & 105 & 110 & 118 & 122 & 134 & 135 & 139 & 140 \\
\hline & 143 & 145 & 152 & 154 & 170 & 180 & 192 & 198 & 205 & 219 & 220 & 225 & 231 & 235 \\
\hline
\end{tabular}




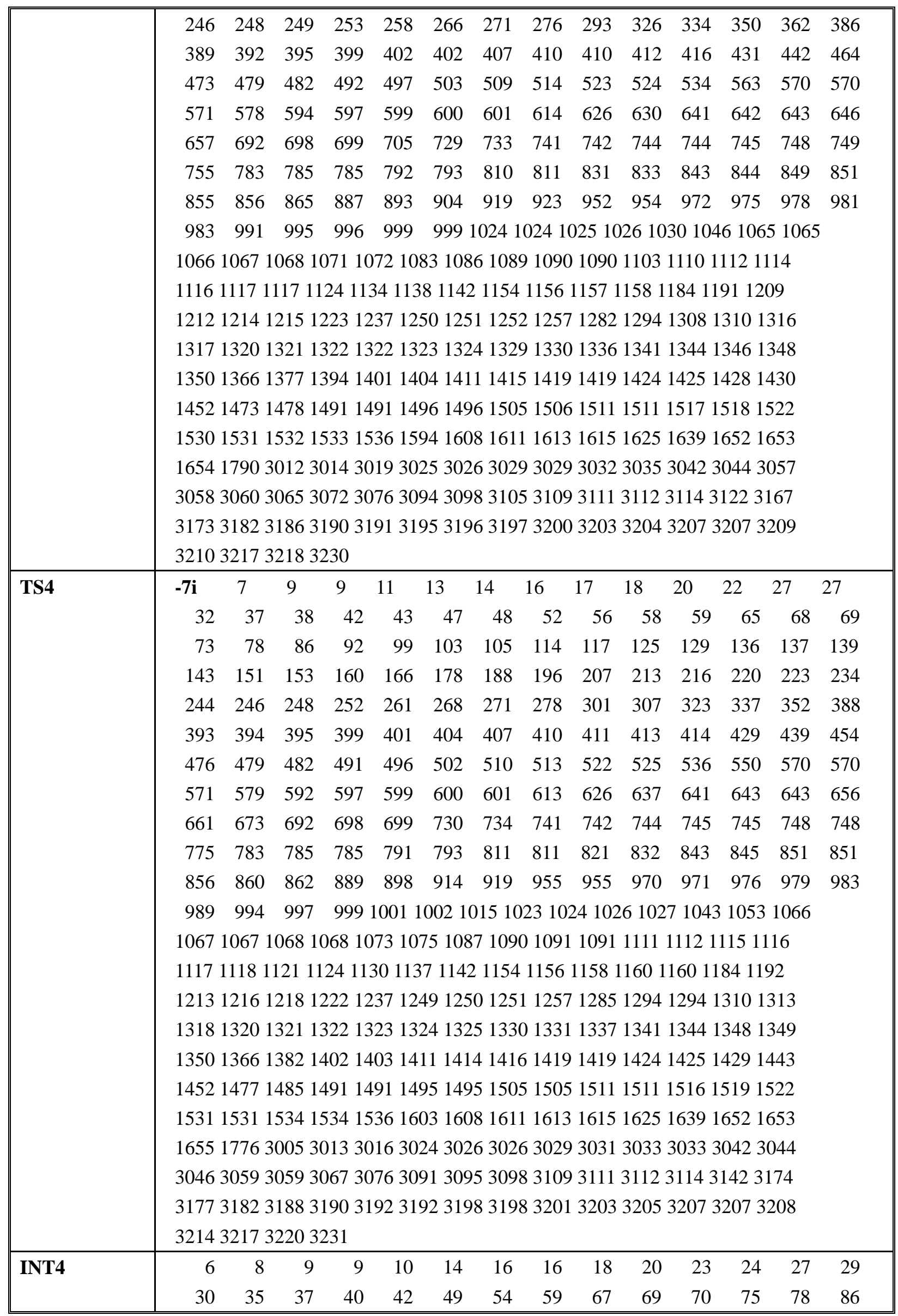




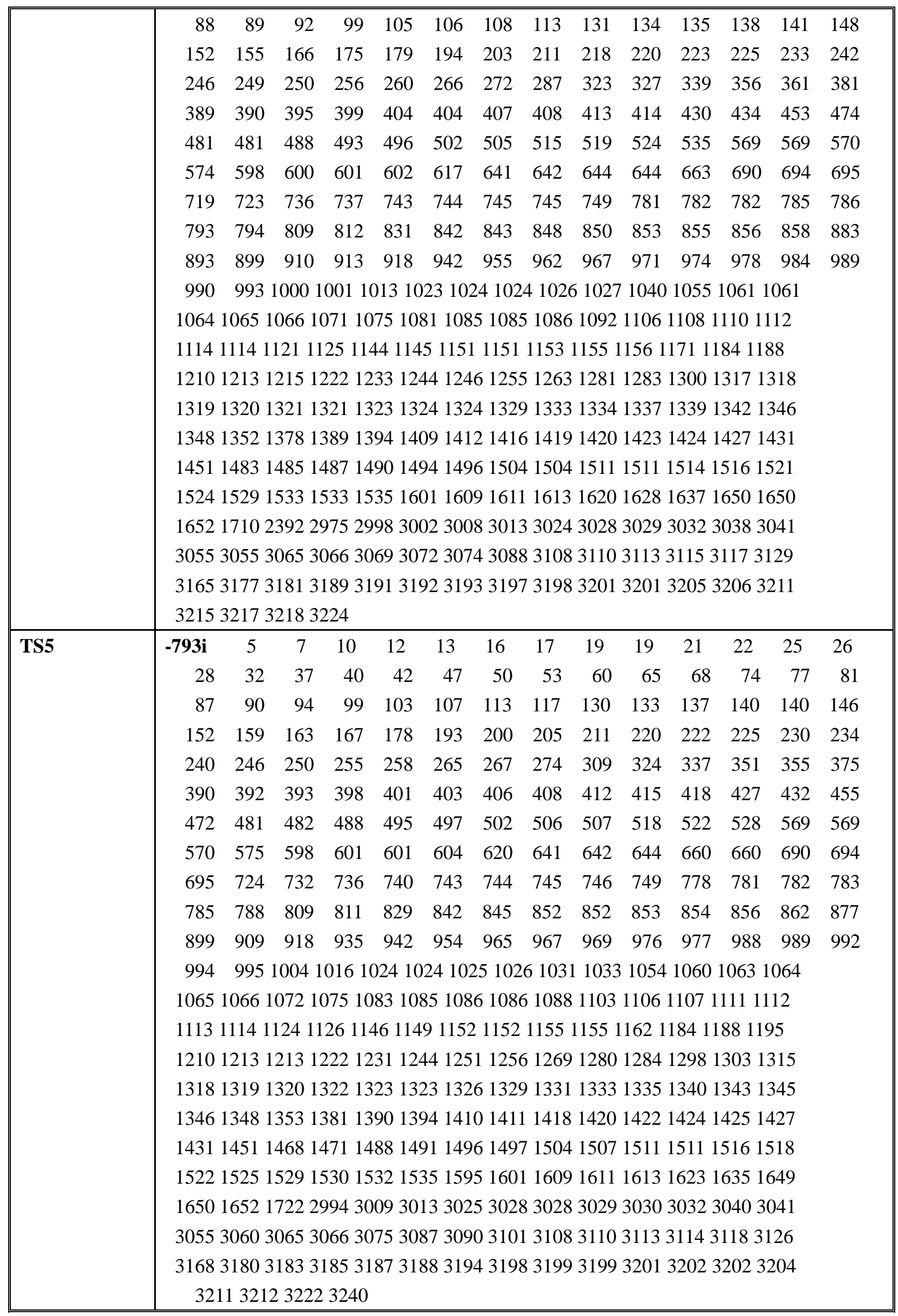




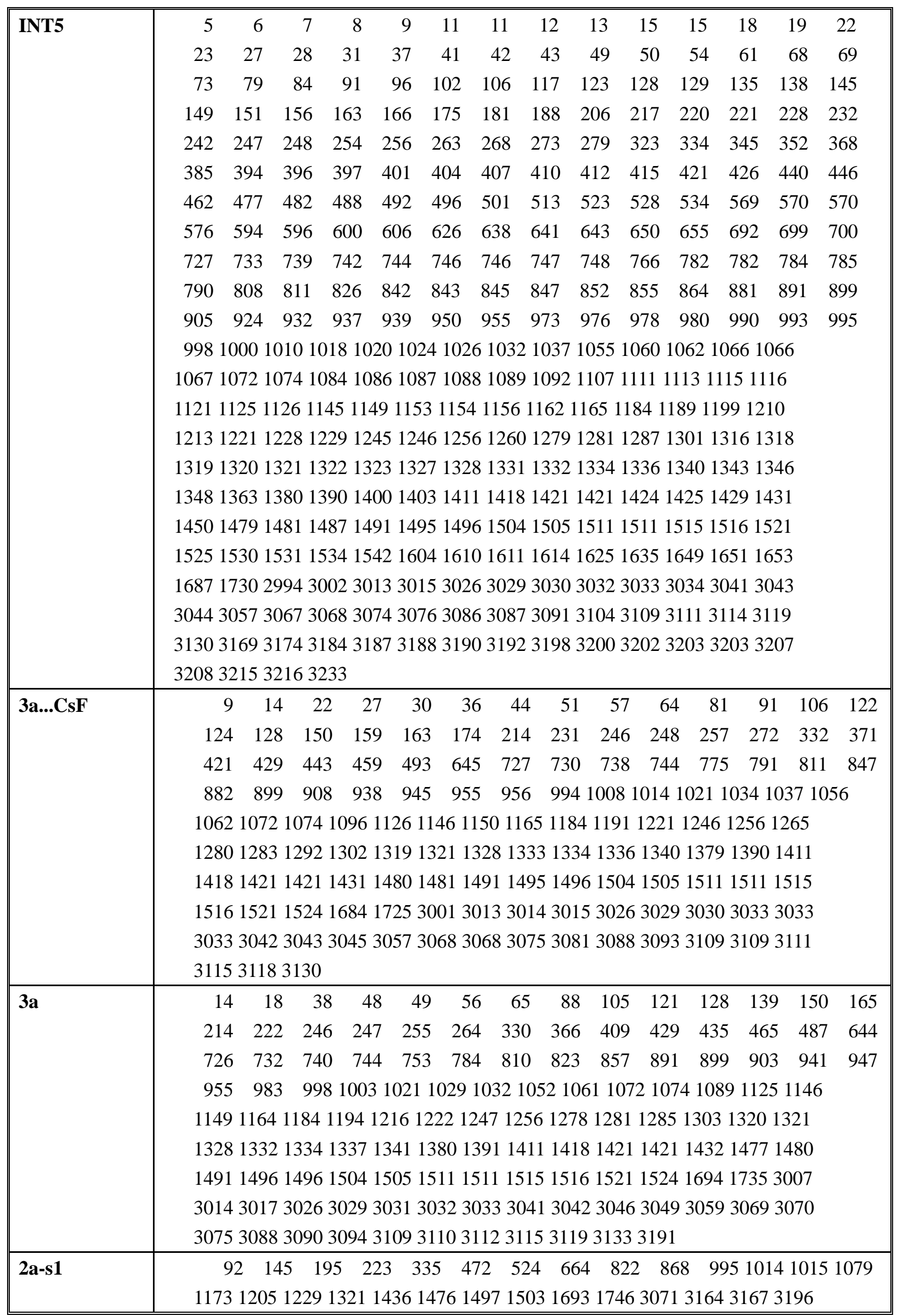




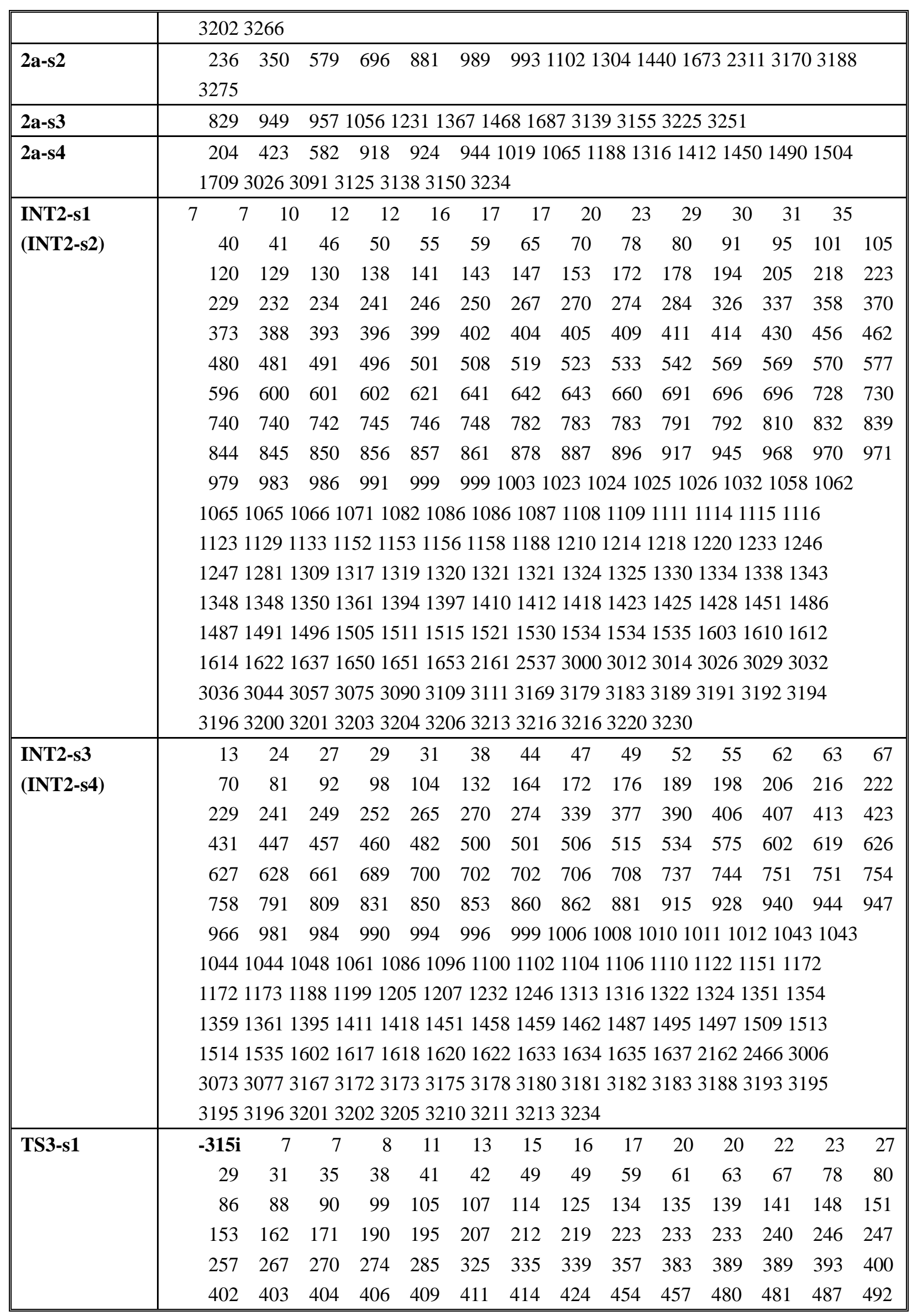




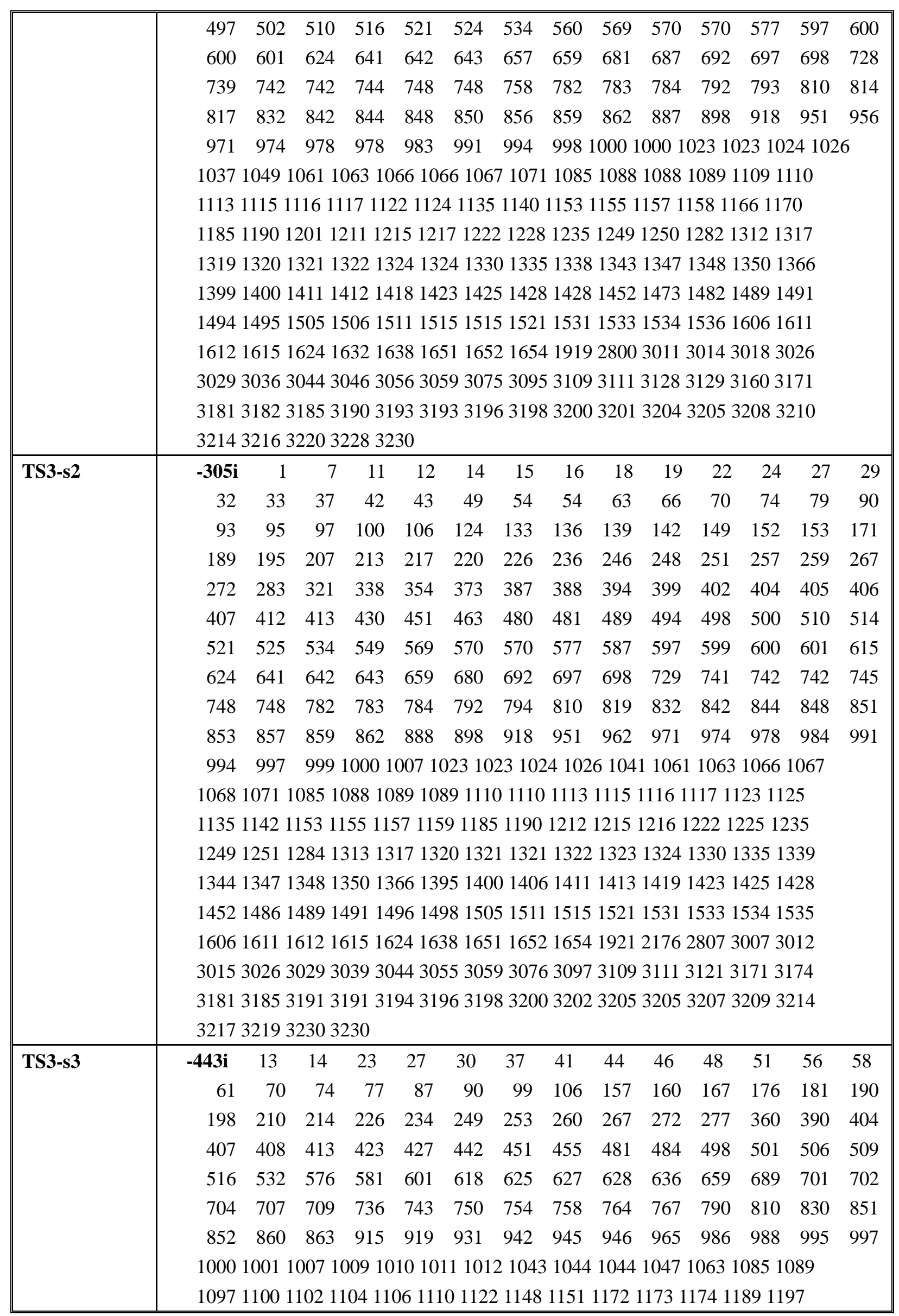




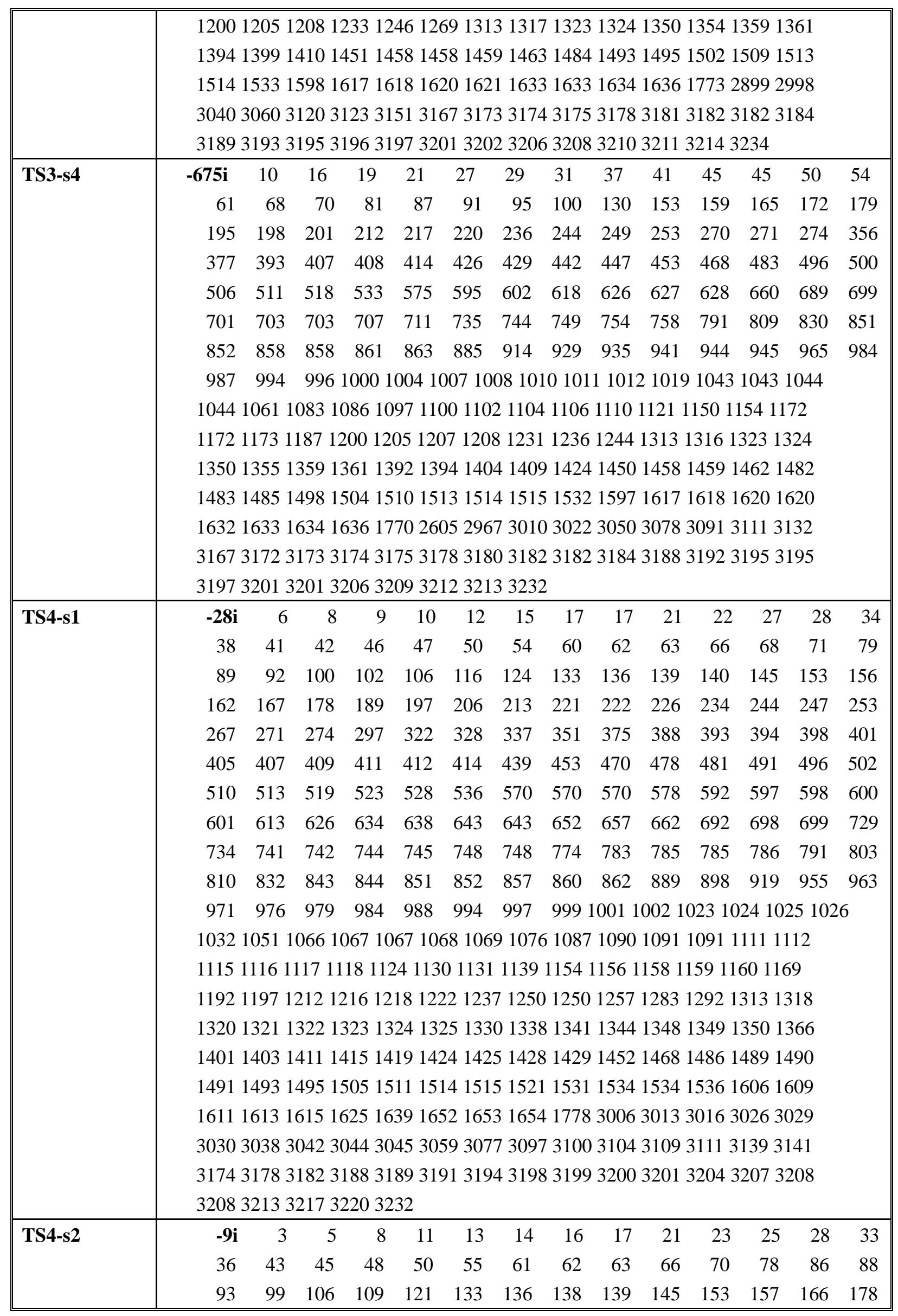

s14 


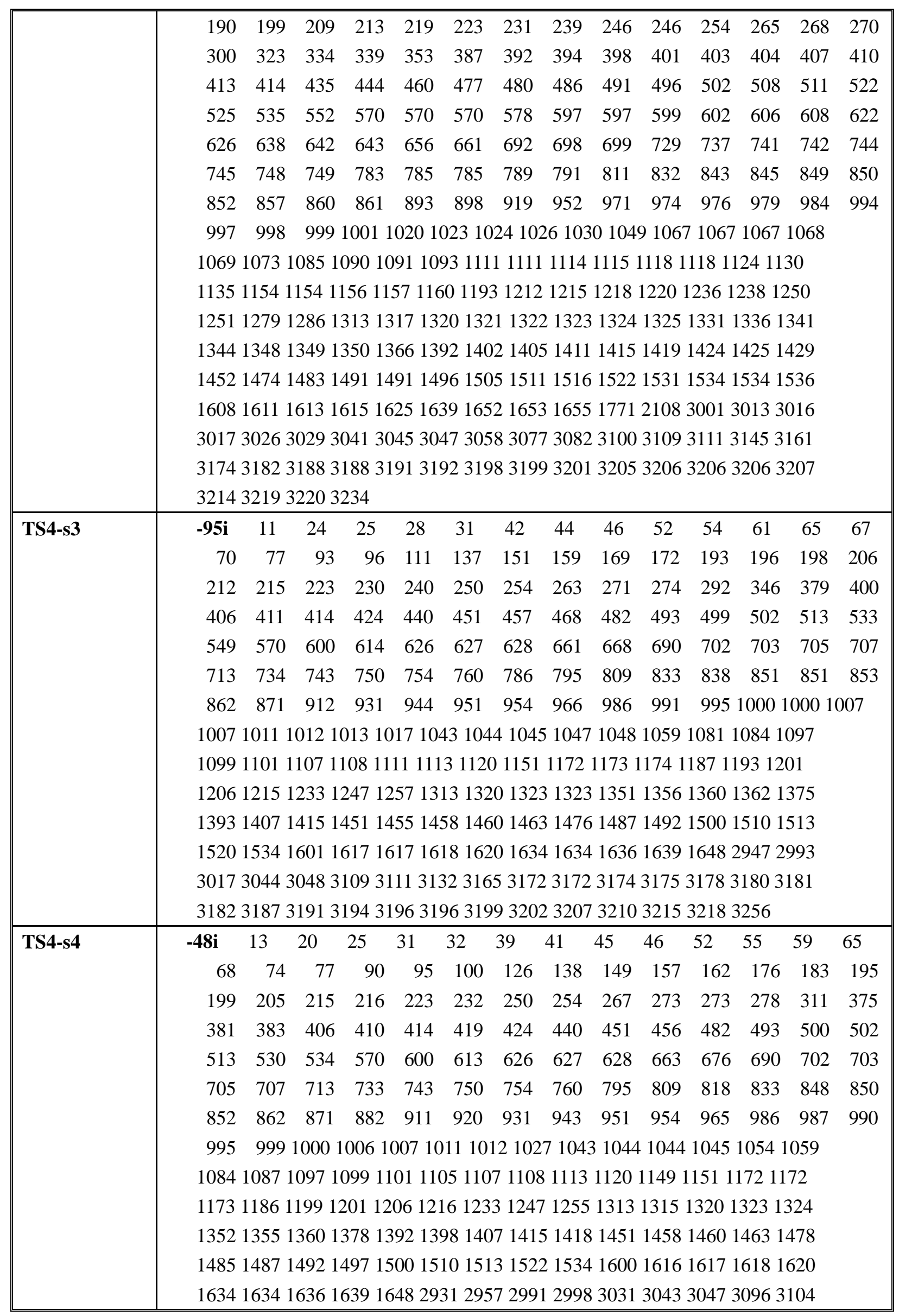


31073164317131723173317531783180318031823187319131943195

31953195319932013207320932133218

Table S1-3. Frequencies of some stationary points for the alternative pathways, computed at IDSCRF (PCM)-B3LYP/DZVP level in DMA.

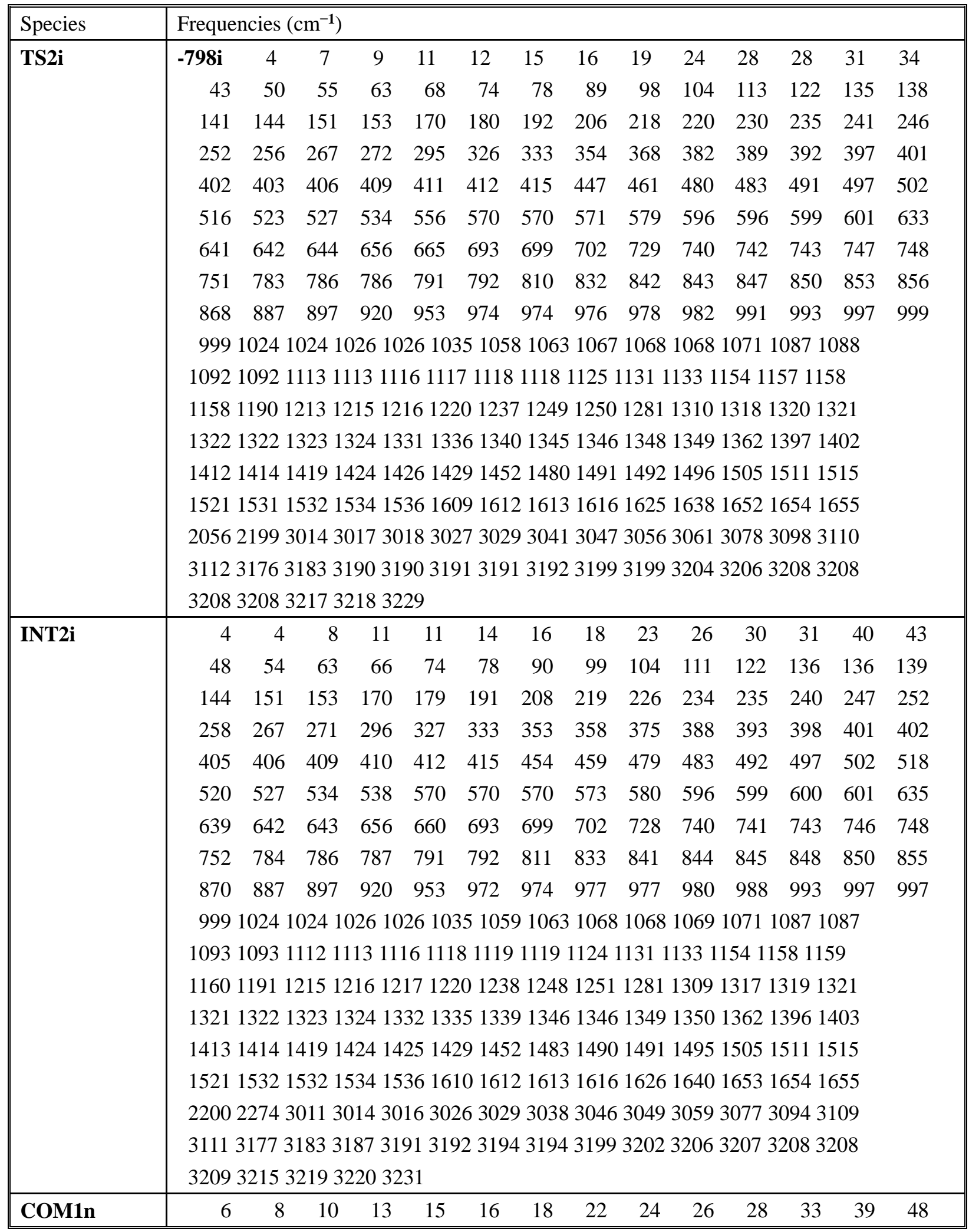




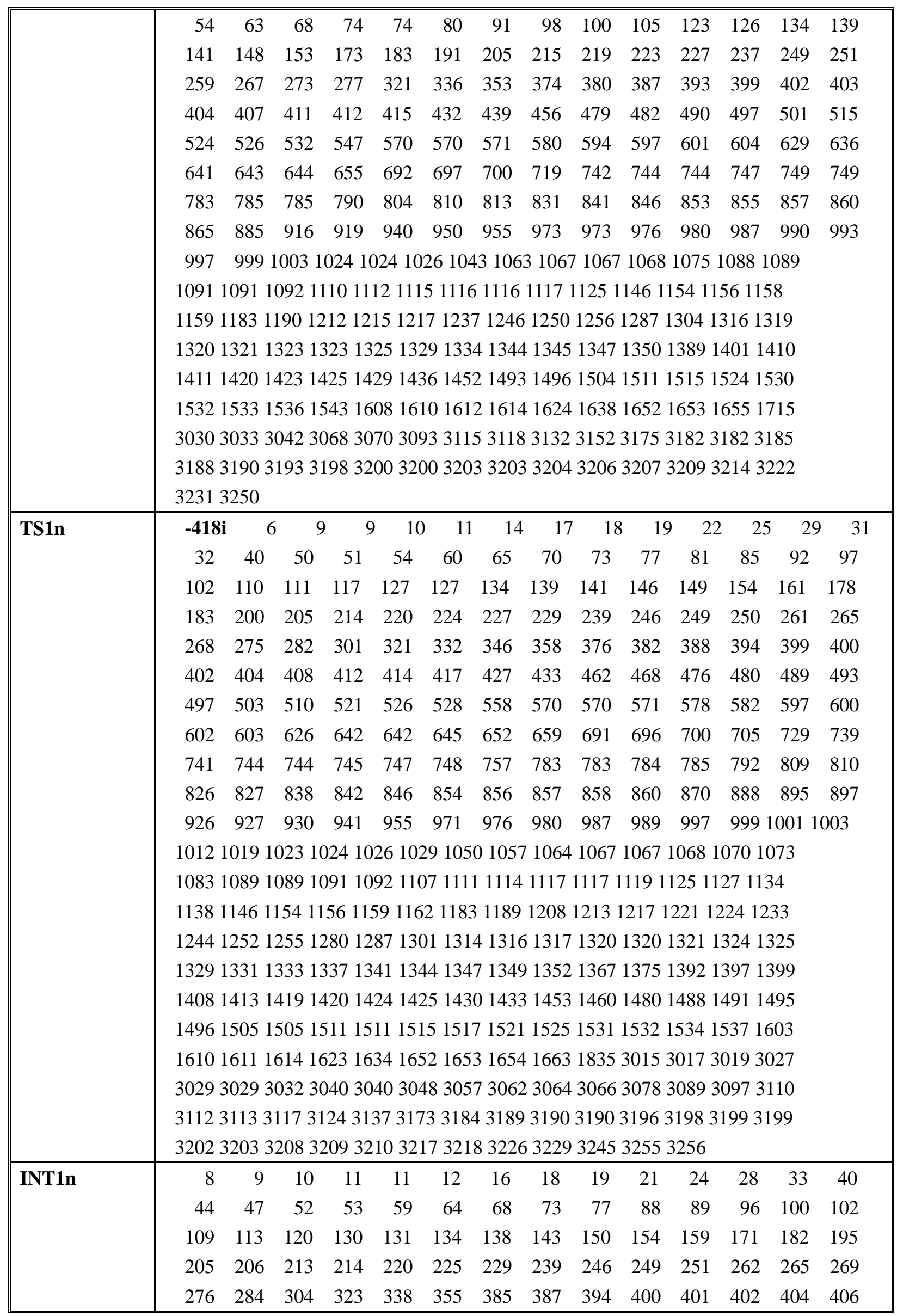




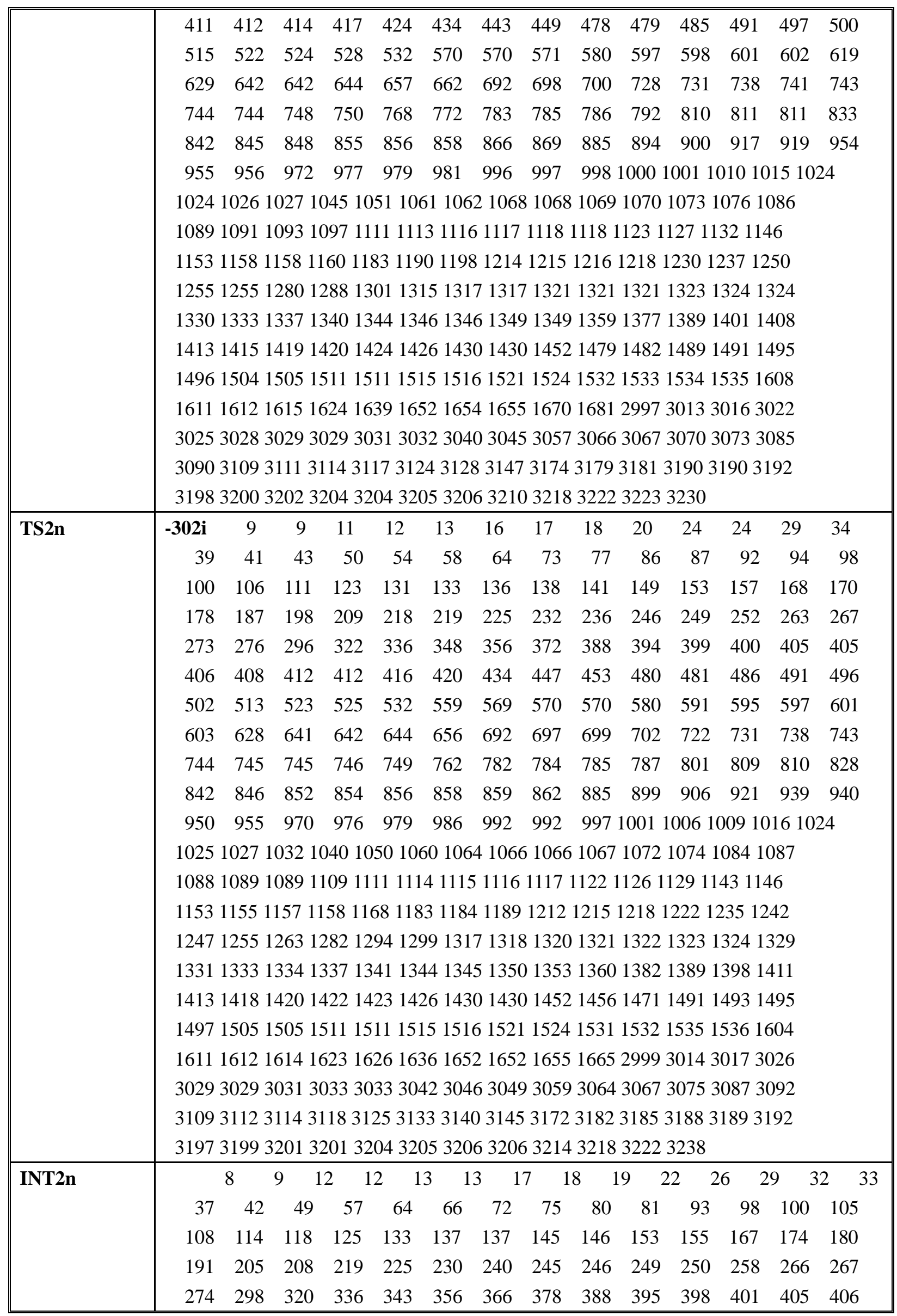




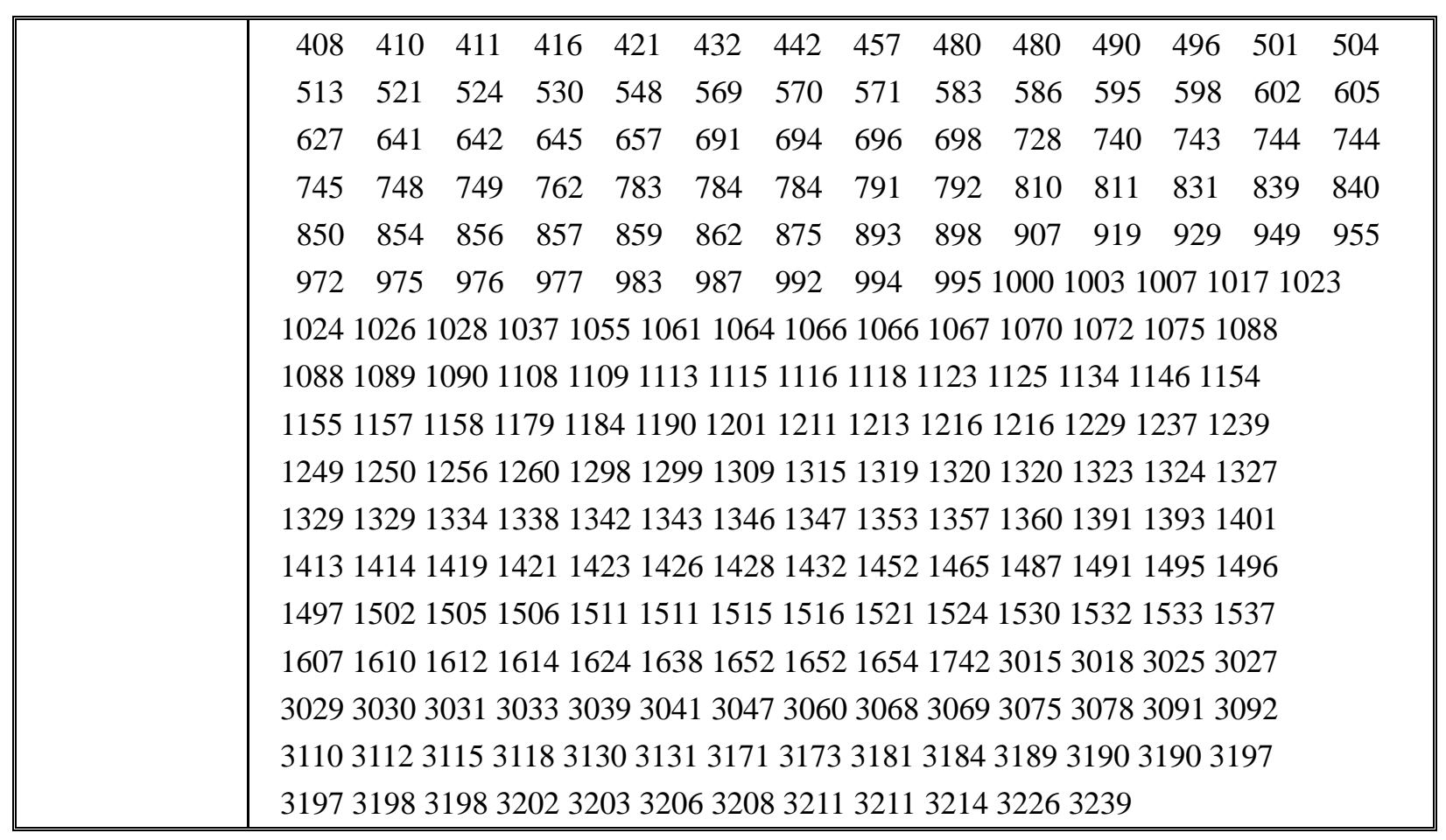

Table S1-4. Frequencies of some stationary points for the rate determining step when phosphine ligand is $\mathrm{P}\left(4-\mathrm{F}_{3} \mathrm{CC}_{6} \mathrm{H}_{4}\right)_{3}, \mathrm{PPh}_{2}\left(\mathrm{C}_{6} \mathrm{~F}_{5}\right)$ or $\mathrm{P}(\mathrm{OMe})_{3}$, computed at IDSCRF (PCM)-B3LYP/DZVP level in DMA.

\begin{tabular}{|c|c|c|c|c|c|c|c|c|c|c|c|c|c|c|}
\hline \multirow{2}{*}{$\begin{array}{l}\text { Species } \\
\text { INT2b }\end{array}$} & \multicolumn{14}{|c|}{ Frequencies $\left(\mathrm{cm}^{-1}\right)$} \\
\hline & 6 & 11 & 18 & 25 & 26 & 28 & 30 & 34 & 39 & 41 & 42 & 48 & 48 & 54 \\
\hline & 55 & 63 & 69 & 72 & 83 & 91 & 96 & 108 & 114 & 126 & 143 & 146 & 162 & 173 \\
\hline & 175 & 196 & 198 & 209 & 224 & 227 & 238 & 243 & 245 & 249 & 253 & 266 & 270 & 274 \\
\hline & 288 & 363 & 374 & 391 & 406 & 407 & 413 & 421 & 428 & 446 & 453 & 456 & 461 & 482 \\
\hline & 500 & 501 & 506 & 515 & 534 & 543 & 575 & 602 & 619 & 626 & 627 & 628 & 661 & 689 \\
\hline & 700 & 702 & 702 & 706 & 708 & 728 & 737 & 740 & 744 & 751 & 754 & 755 & 758 & 791 \\
\hline & 792 & 809 & 831 & 850 & 853 & 860 & 862 & 886 & 887 & 896 & 916 & 928 & 941 & 944 \\
\hline & 947 & 966 & 969 & 983 & 990 & 994 & 996 & 999 & 999 & 10061 & 0081 & 1010 & 1110 & \\
\hline & \multirow{8}{*}{\multicolumn{14}{|c|}{$\begin{array}{l}10251032104310441044105810611071108610961100110211041106 \\
11101122112911331151117211721173118811991205120812201232 \\
12461247128013091313131613191323132513331338135113541359 \\
13611361139413951412141214181451145814591462148614871491 \\
14961505150915111513151415151521153516021617161816201621 \\
16331634163516372157245329943012301430253029302930333044 \\
30563074308831093111316731733174317531783180318131823183 \\
318831933195319531973201320232073210321132123233\end{array}$}} \\
\hline & & & & & & & & & & & & & & \\
\hline & & & & & & & & & & & & & & \\
\hline & & & & & & & & & & & & & & \\
\hline & & & & & & & & & & & & & & \\
\hline & & & & & & & & & & & & & & \\
\hline & & & & & & & & & & & & & & \\
\hline & & & & & & & & & & & & & & \\
\hline \multirow[t]{5}{*}{ TS3b } & $-327 i$ & 8 & 9 & 15 & 17 & 21 & 25 & 26 & 28 & 32 & 32 & 37 & 39 & 41 \\
\hline & 43 & 46 & 47 & 53 & 57 & 58 & 65 & 68 & 71 & 75 & 77 & 84 & 88 & 93 \\
\hline & 96 & 99 & 107 & 122 & 124 & 136 & 143 & 152 & 153 & 163 & 171 & 189 & 190 & 197 \\
\hline & 209 & 217 & 224 & 229 & 234 & 246 & 249 & 249 & 252 & 258 & 261 & 268 & 270 & 273 \\
\hline & 284 & 372 & 386 & 391 & 402 & 405 & 407 & 413 & 428 & 430 & 433 & 440 & 452 & 456 \\
\hline
\end{tabular}




\begin{tabular}{|c|c|c|c|c|c|c|c|c|c|c|c|c|c|c|}
\hline & 463 & 481 & 494 & 501 & 502 & 509 & 515 & 533 & 554 & 572 & 577 & 600 & 622 & 626 \\
\hline & 627 & 628 & 632 & 660 & 676 & 690 & 699 & 702 & 703 & 704 & 707 & 710 & 728 & 740 \\
\hline & 741 & 744 & 747 & 750 & 754 & 758 & 768 & 793 & 793 & 810 & 810 & 826 & 833 & 851 \\
\hline & 853 & 859 & 860 & 862 & 887 & 895 & 901 & 917 & 929 & 930 & 941 & 945 & 954 & 955 \\
\hline & 964 & 969 & 986 & 994 & 994 & 996 & 9991 & 10021 & 0081 & 00910 & 11101 & 11101 & 2102 ? & \\
\hline & \multirow{11}{*}{\multicolumn{14}{|c|}{$\begin{array}{l}10271037104310441044105910631066107110751087109811011102 \\
11051107110711111123113511371143115211591173117311741184 \\
11891201120412071222122812351249125012541272128212981312 \\
13141314131713201323132413311334133813501355135913601364 \\
13841397139914081412141314181420143714521459146014631482 \\
14891491149514961504150515051510151115111513151315151518 \\
15211528153616041610161816191621162316331634163616381922 \\
27423010301330163025302730293031303430363043304630543057 \\
30633075308130973104310831113113311531413170317431763176 \\
31773180318331833184318531913195319531973198320232043205 \\
32073209321232323234\end{array}$}} \\
\hline & & & & & & & & & & & & & & \\
\hline & & & & & & & & & & & & & & \\
\hline & & & & & & & & & & & & & & \\
\hline & & & & & & & & & & & & & & \\
\hline & & & & & & & & & & & & & & \\
\hline & & & & & & & & & & & & & & \\
\hline & & & & & & & & & & & & & & \\
\hline & & & & & & & & & & & & & & \\
\hline & & & & & & & & & & & & & & \\
\hline & & & & & & & & & & & & & & \\
\hline \multirow[t]{21}{*}{ INT3b } & 5 & 9 & 15 & 16 & 18 & 24 & 26 & 31 & 33 & 35 & 36 & 38 & 42 & 45 \\
\hline & 45 & 47 & 51 & 53 & 57 & 61 & 65 & 67 & 70 & 73 & 78 & 83 & 87 & 96 \\
\hline & 101 & 110 & 120 & 123 & 138 & 140 & 144 & 154 & 161 & 172 & 183 & 191 & 194 & 196 \\
\hline & 213 & 221 & 229 & 231 & 246 & 246 & 249 & 250 & 254 & 259 & 270 & 274 & 275 & 292 \\
\hline & 361 & 387 & 392 & 405 & 406 & 409 & 412 & 429 & 432 & 441 & 443 & 452 & 467 & 475 \\
\hline & 480 & 502 & 503 & 509 & 513 & 517 & 532 & 567 & 577 & 597 & 602 & 624 & 626 & 627 \\
\hline & 628 & 630 & 638 & 648 & 659 & 691 & 703 & 704 & 705 & 706 & 707 & 729 & 733 & 735 \\
\hline & 742 & 743 & 744 & 748 & 751 & 754 & 757 & 777 & 793 & 793 & 810 & 810 & 829 & 833 \\
\hline & 853 & 858 & 861 & 863 & 888 & 892 & 904 & 918 & 922 & 933 & 939 & 945 & 954 & 957 \\
\hline & 971 & 984 & 988 & 992 & 995 & 996 & 9991 & 10041 & 0081 & 00910 & 1010 & 11101 & 2102 & \\
\hline & 1031 & 10431 & 0441 & 0441 & 4710 & 54106 & 61071 & 11073 & 1083 & 1089 & 10991 & 10011 & 101 & \\
\hline & 1102 & 11061 & 10711 & 11211 & 24113 & 41138 & 81142 & 1153 & 1173 & 1741 & 17411 & 8411 & & \\
\hline & 1202 & 12041 & 2061 & 2101 & $2412 ?$ & 36125 & 01251 & 11252 & 1258 & 1283 & 12941 & 30913 & 310 & \\
\hline & 1315 & 13161 & 3171 & 3231 & 2413 & 24132 & 91336 & 61342 & 1350 & 1357 & 13581 & 36013 & 366 & \\
\hline & 1377 & 13931 & 4001 & 4051 & 1314 & 15141 & 91419 & 91429 & 1452 & 1459 & 14601 & 4631 & 473 & \\
\hline & 1479 & 14901 & 4911 & 4961 & 9615 & 5150 & 71511 & 11511 & 1511 & 15121 & 5131 & 51715 & & \\
\hline & 1522 & 15301 & 5361 & 5971 & 0816 & 18161 & 91621 & 11624 & 1633 & 1634 & 16361 & 63917 & 783 & \\
\hline & 3011 & 30143 & 0173 & 0243 & 25302 & 27302 & 93031 & 13032 & 3034 & $3042 ?$ & 30443 & 05330 & 057 & \\
\hline & 3060 & 30633 & 0723 & 0763 & 9430 & 98310 & 93111 & 13111 & 3113 & 31143 & 1653 & 17231 & & \\
\hline & 3177 & 31783 & 1813 & 1843 & 84318 & 36318 & 63192 & 23195 & 3196 & 3196 & 31983 & 2033 & 203 & \\
\hline & 3203 & 32063 & 2103 & 2113 & & & & & & & & & & \\
\hline \multirow[t]{7}{*}{ TS4b } & $-14 i$ & 6 & 12 & 14 & 18 & 23 & 26 & 29 & 33 & 37 & 37 & 40 & 40 & 43 \\
\hline & 44 & 46 & 49 & 53 & 58 & 60 & 64 & 67 & 69 & 73 & 78 & 85 & 88 & 94 \\
\hline & 105 & 113 & 116 & 130 & 133 & 138 & 150 & 155 & 162 & 166 & 177 & 187 & 194 & 199 \\
\hline & 213 & 218 & 224 & 231 & 237 & 246 & 248 & 249 & 253 & 256 & 270 & 272 & 275 & 304 \\
\hline & 355 & 382 & 394 & 406 & 406 & 409 & 413 & 428 & 430 & 436 & 439 & 450 & 457 & 479 \\
\hline & 481 & 498 & 501 & 507 & 509 & 517 & 534 & 577 & 579 & 597 & 604 & 612 & 624 & 625 \\
\hline & 627 & 627 & 632 & 655 & 657 & 669 & 691 & 704 & 705 & 705 & 707 & 708 & 729 & 730 \\
\hline
\end{tabular}




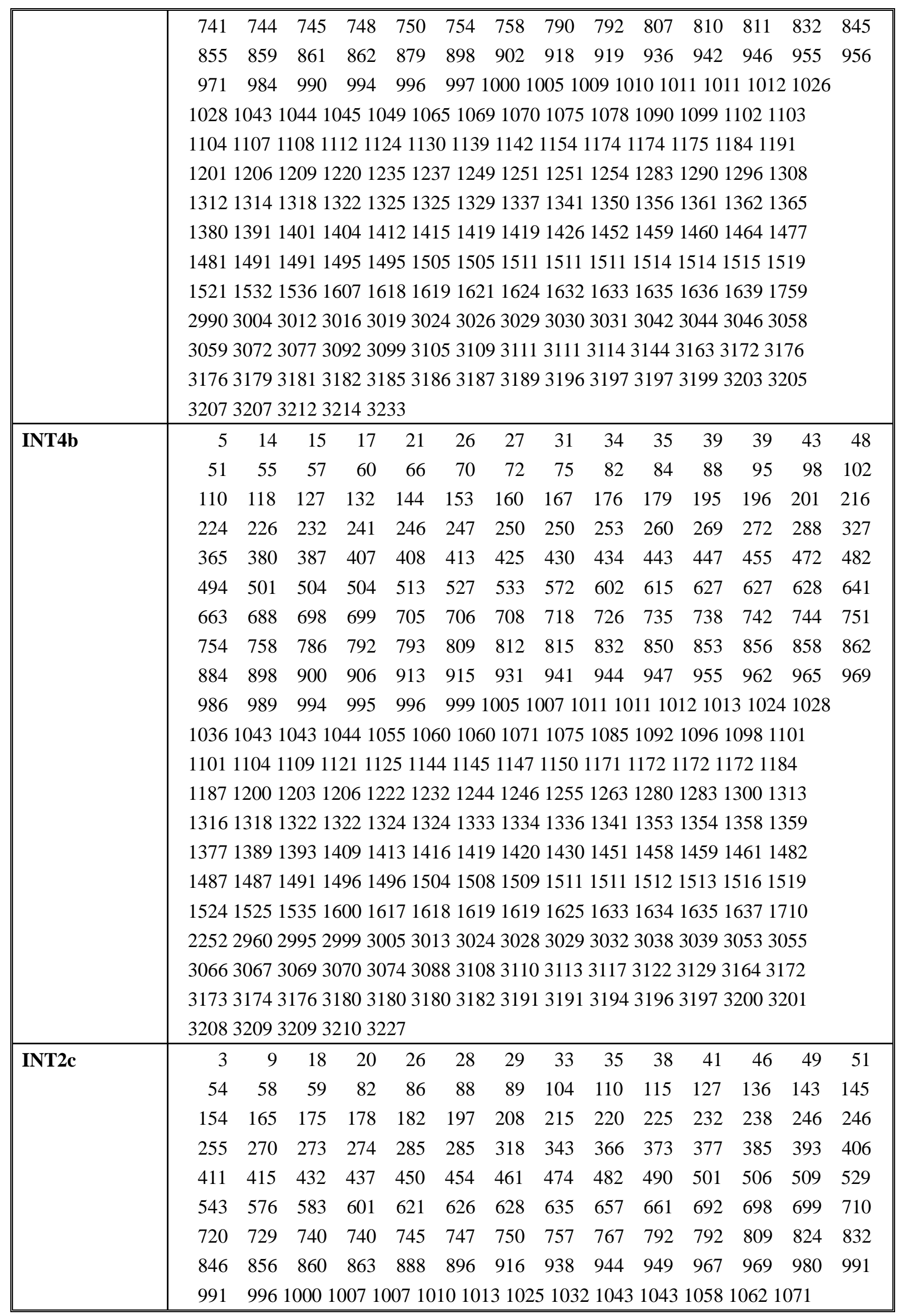




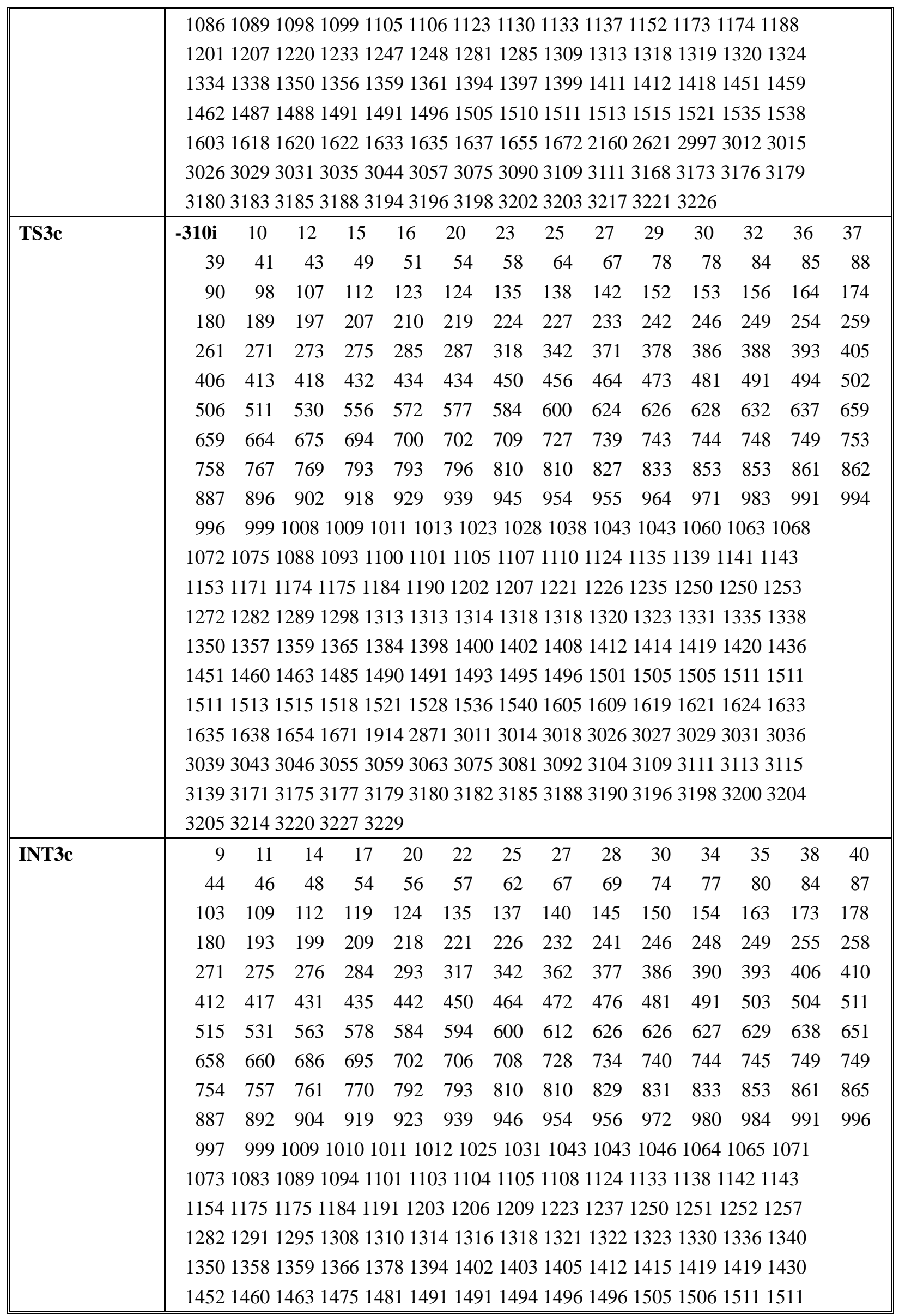









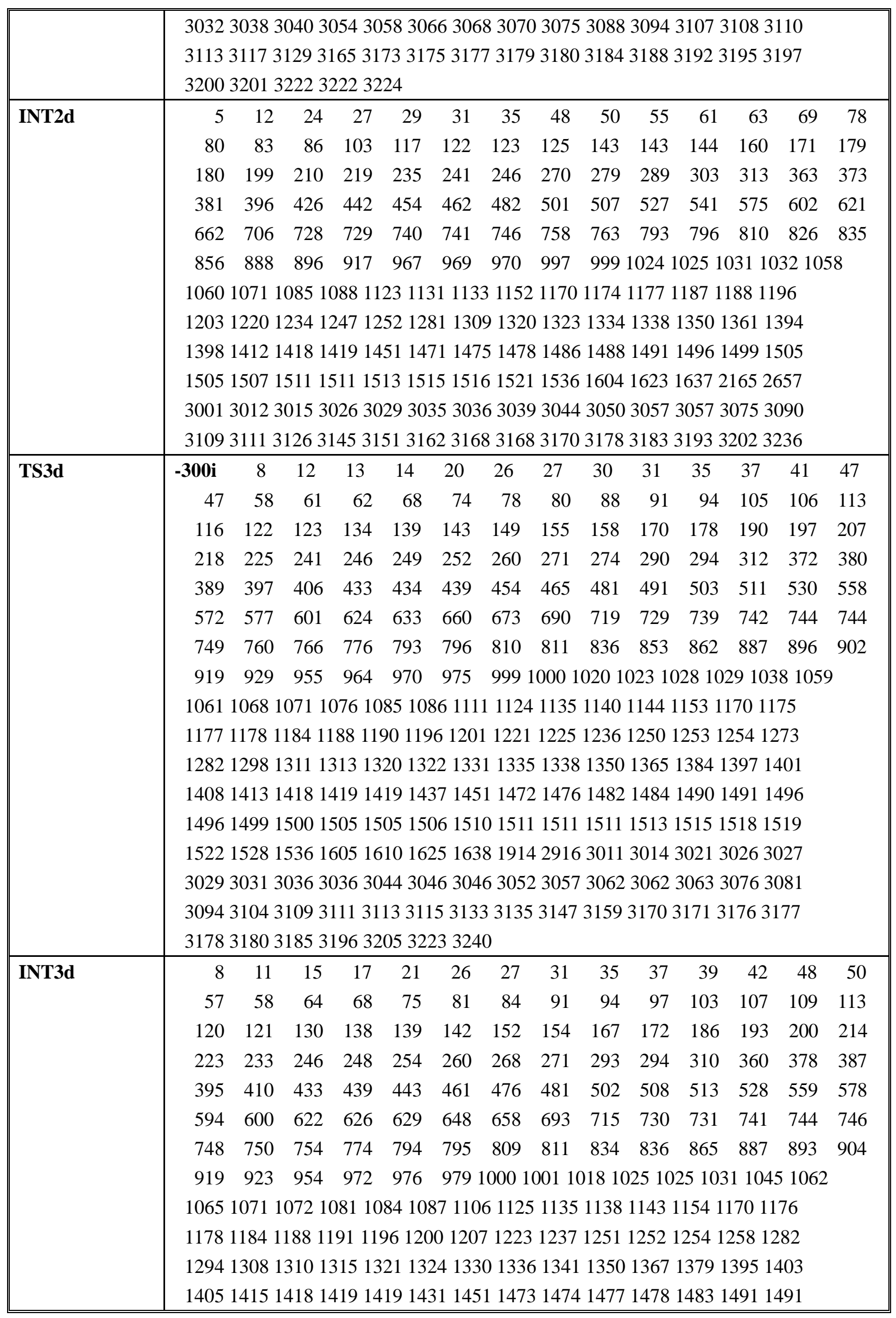




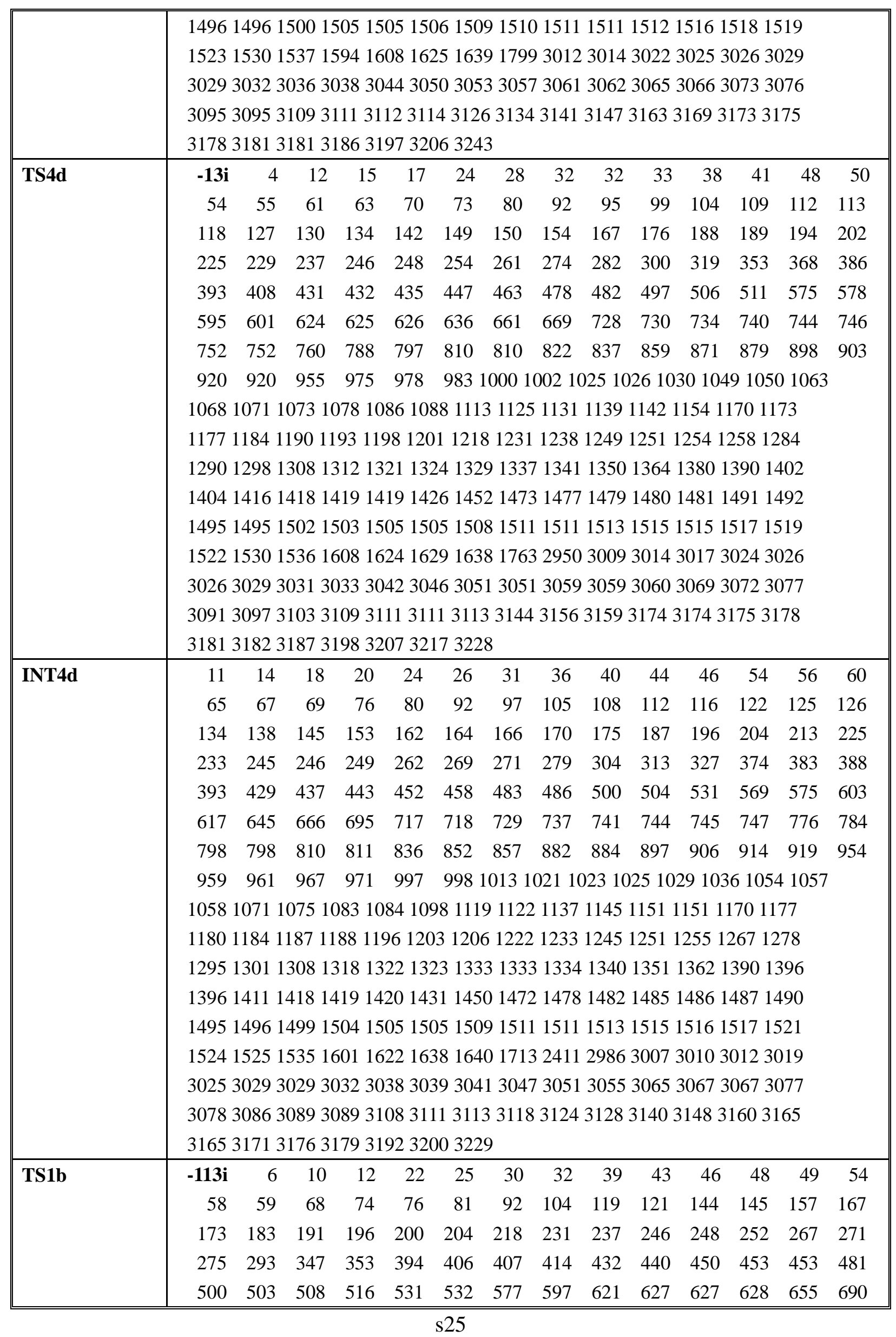




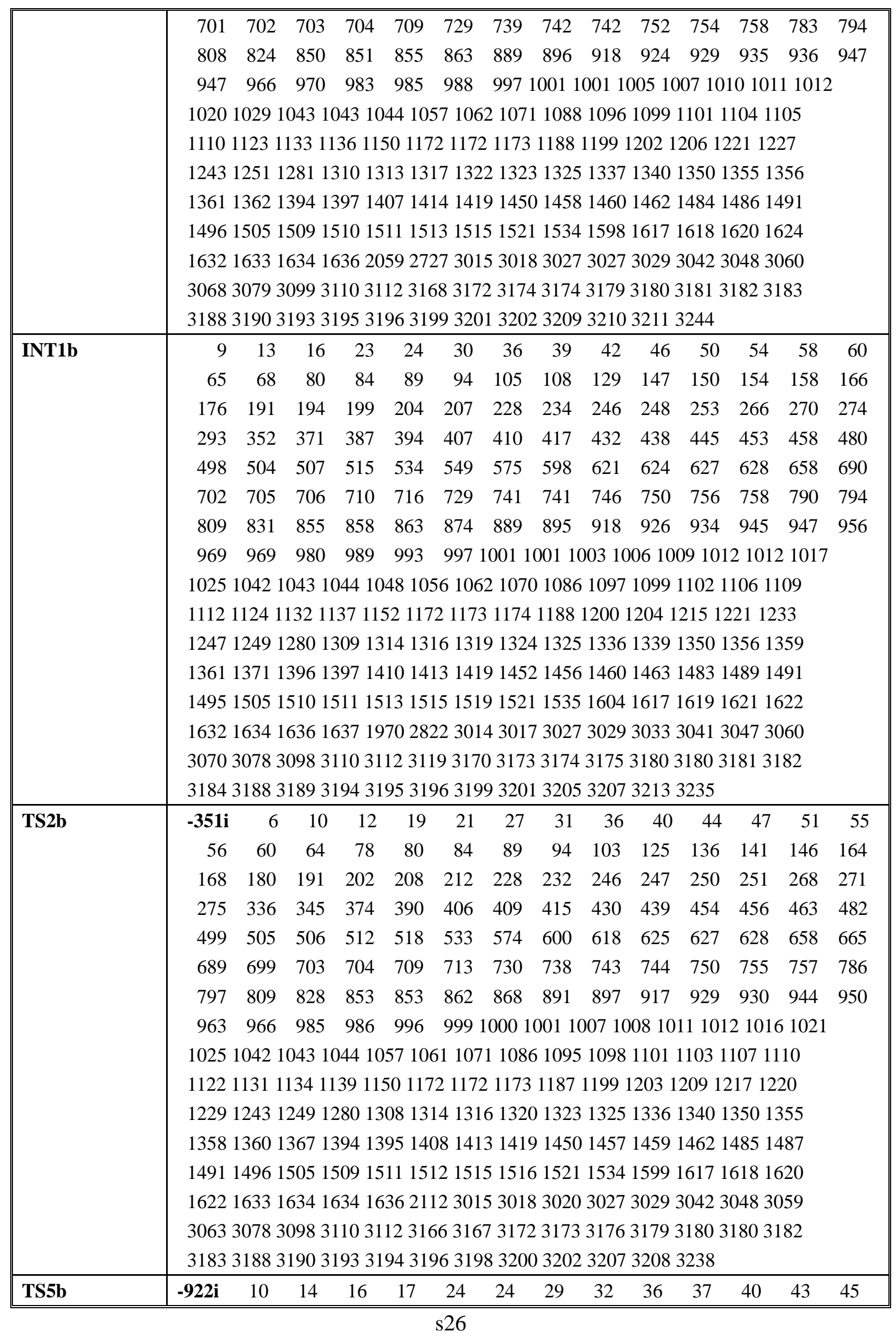




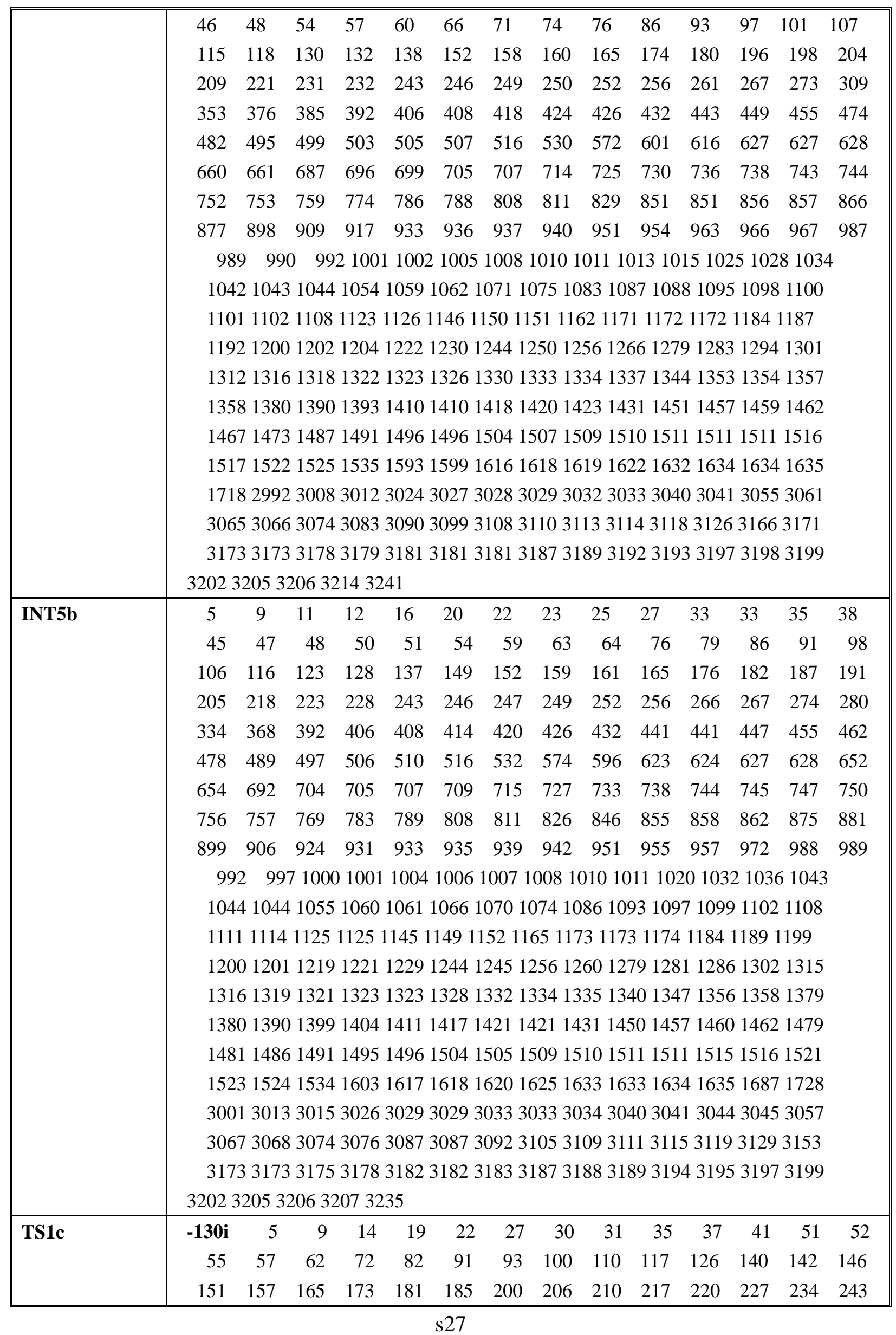




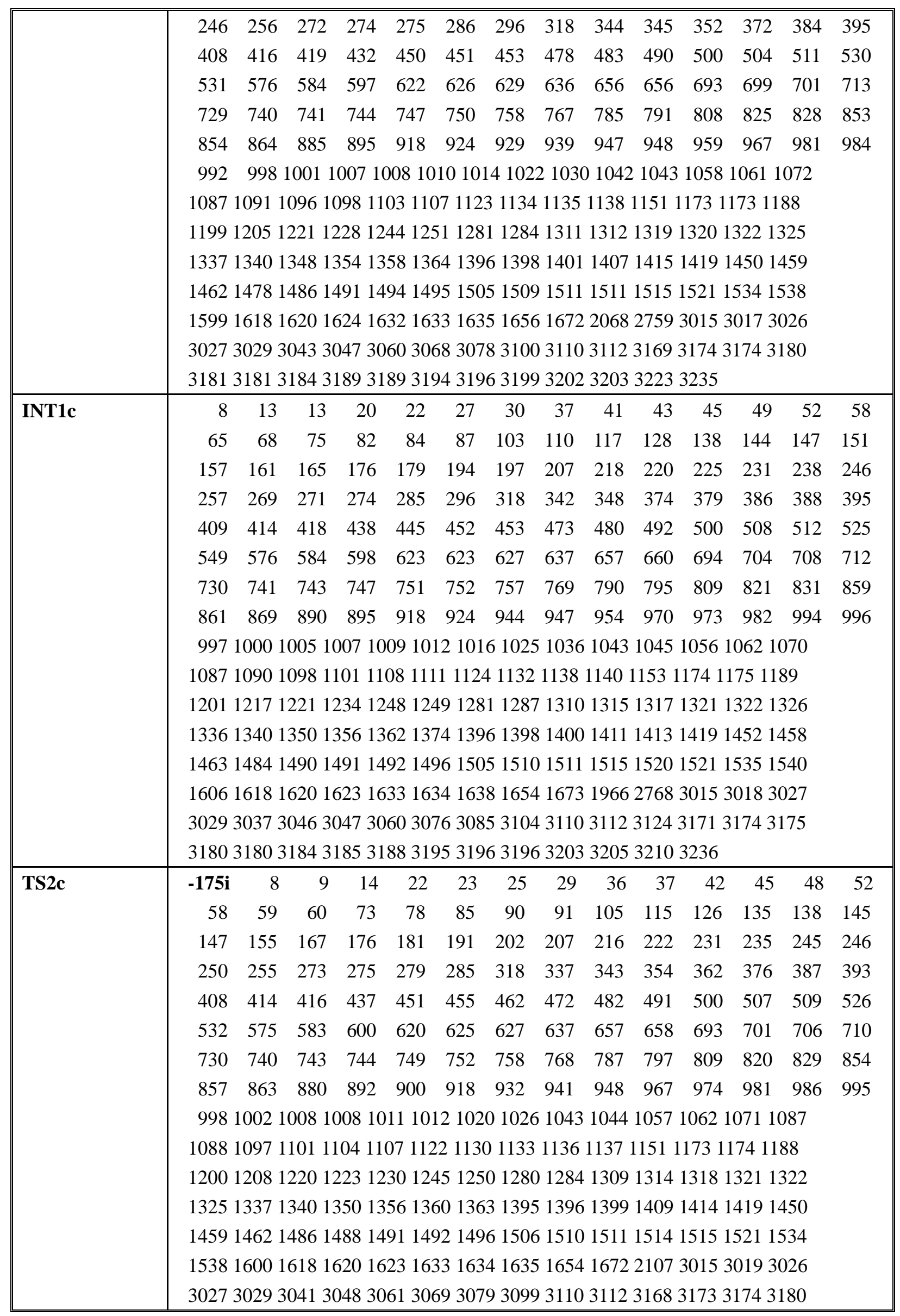




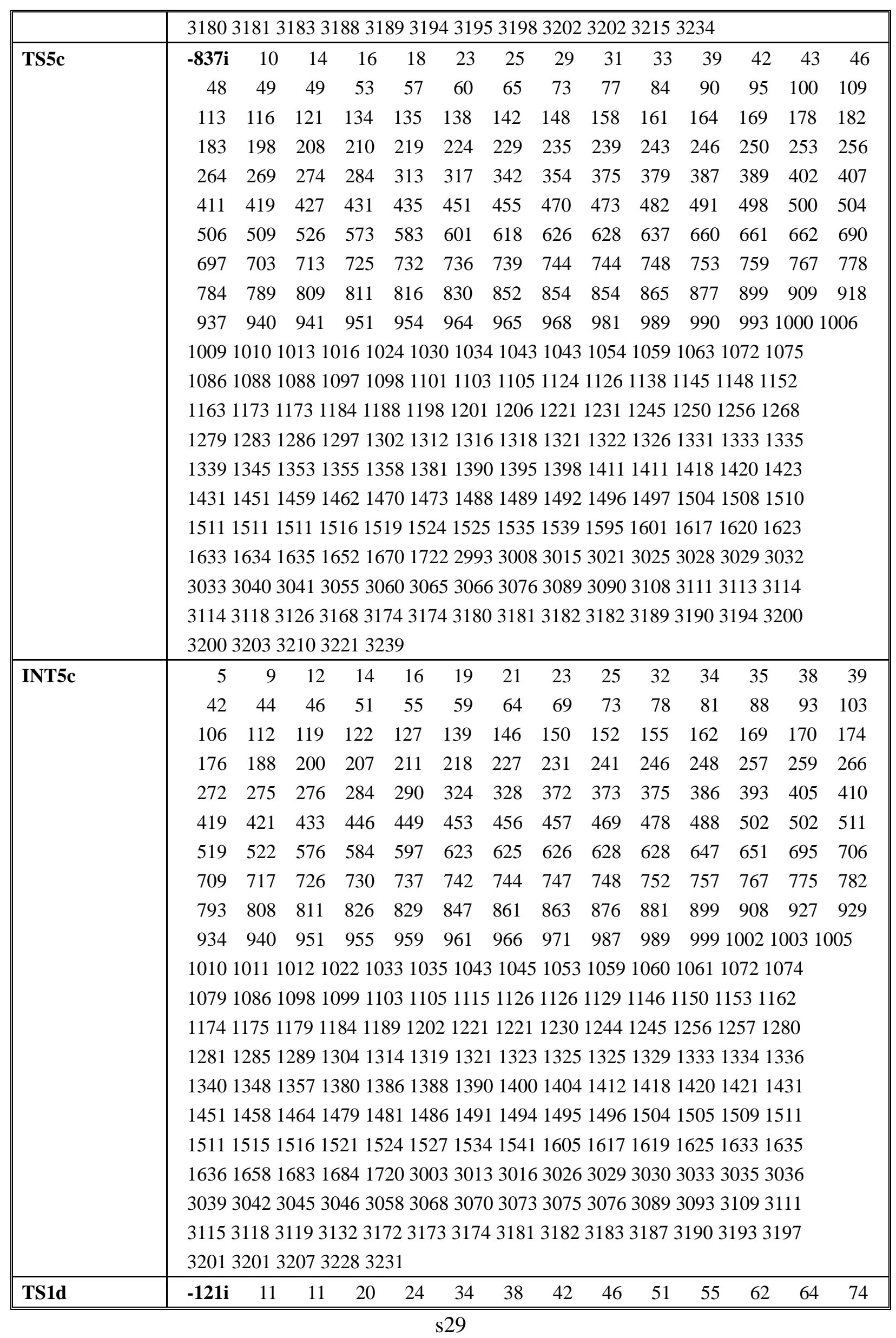




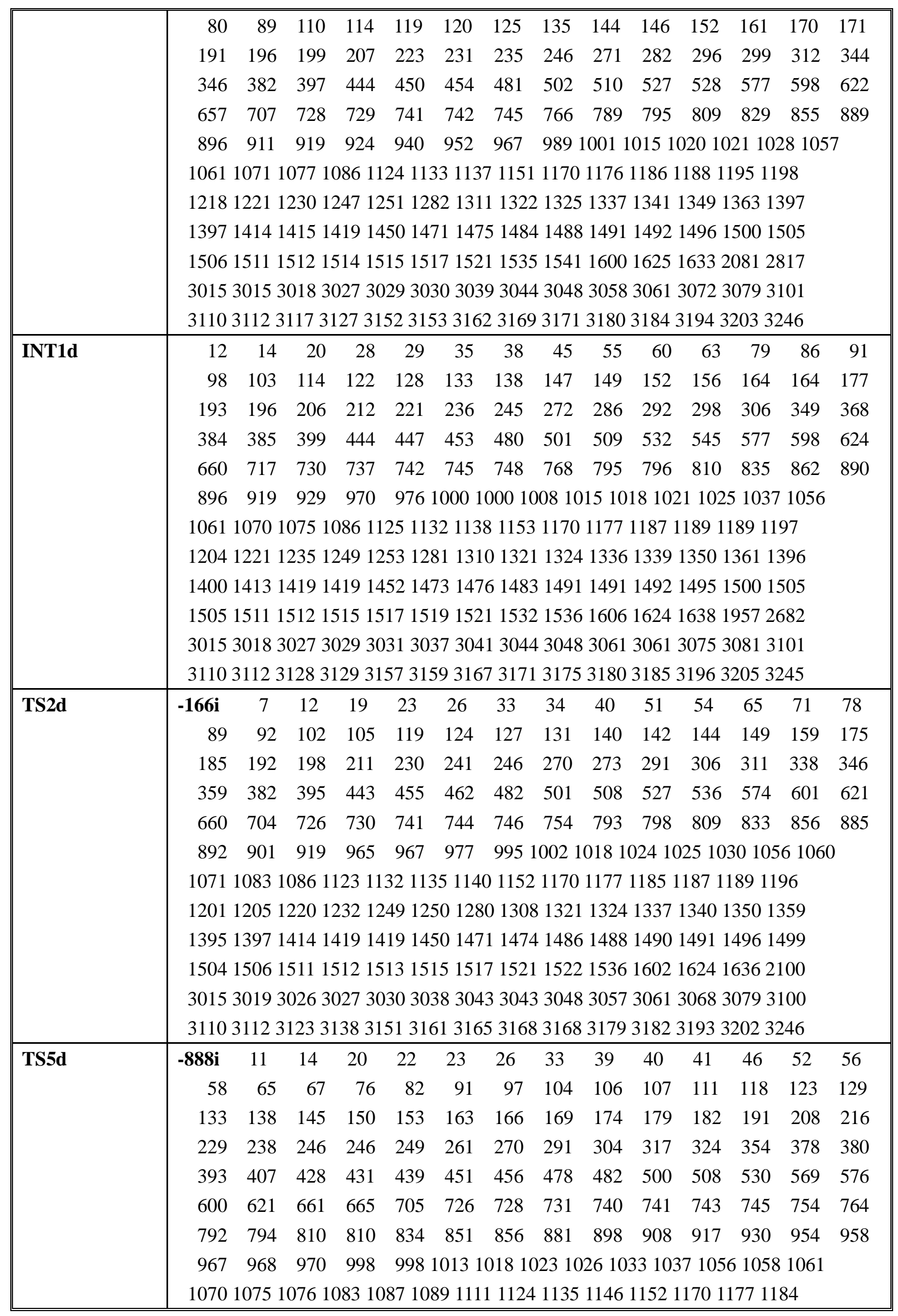




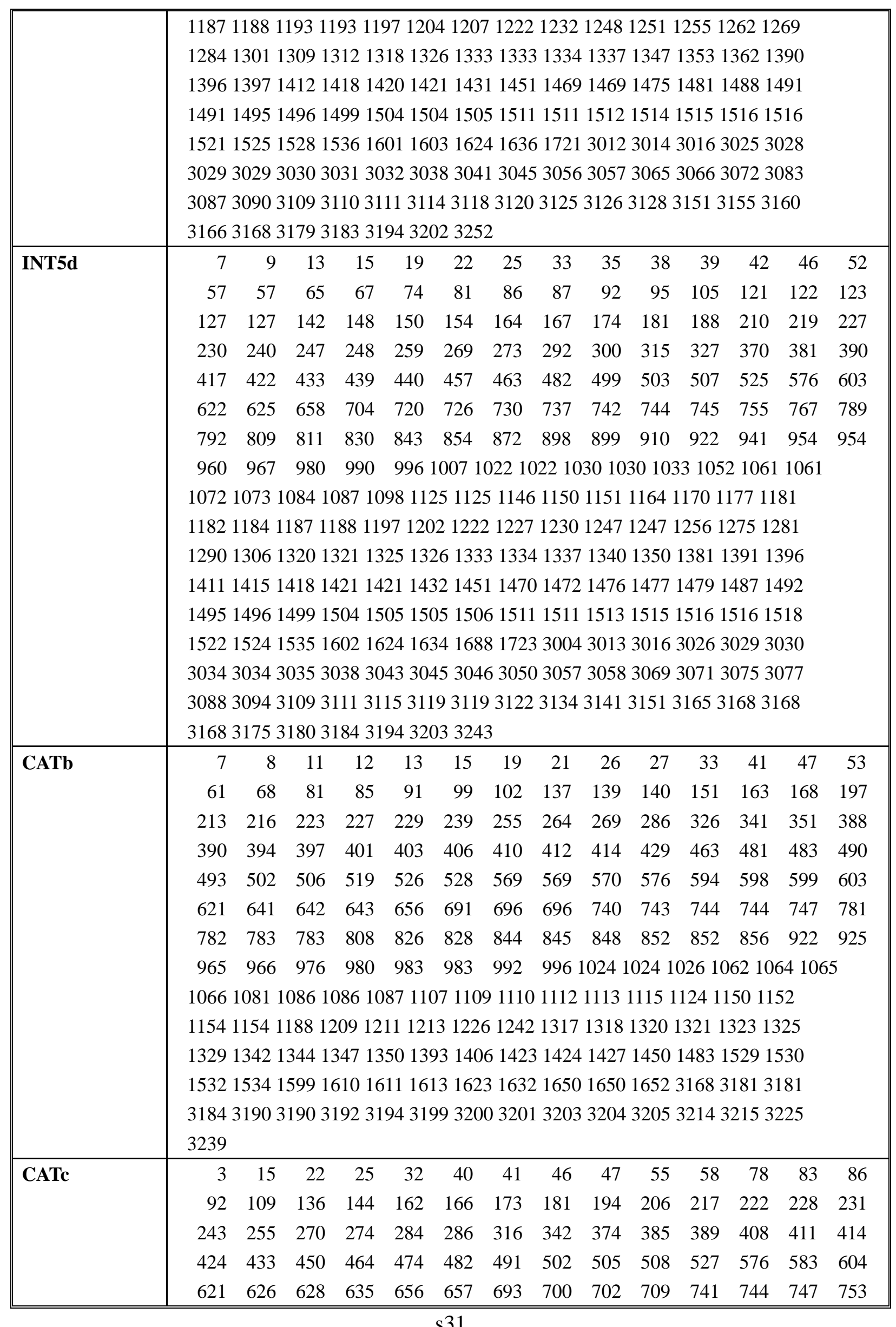




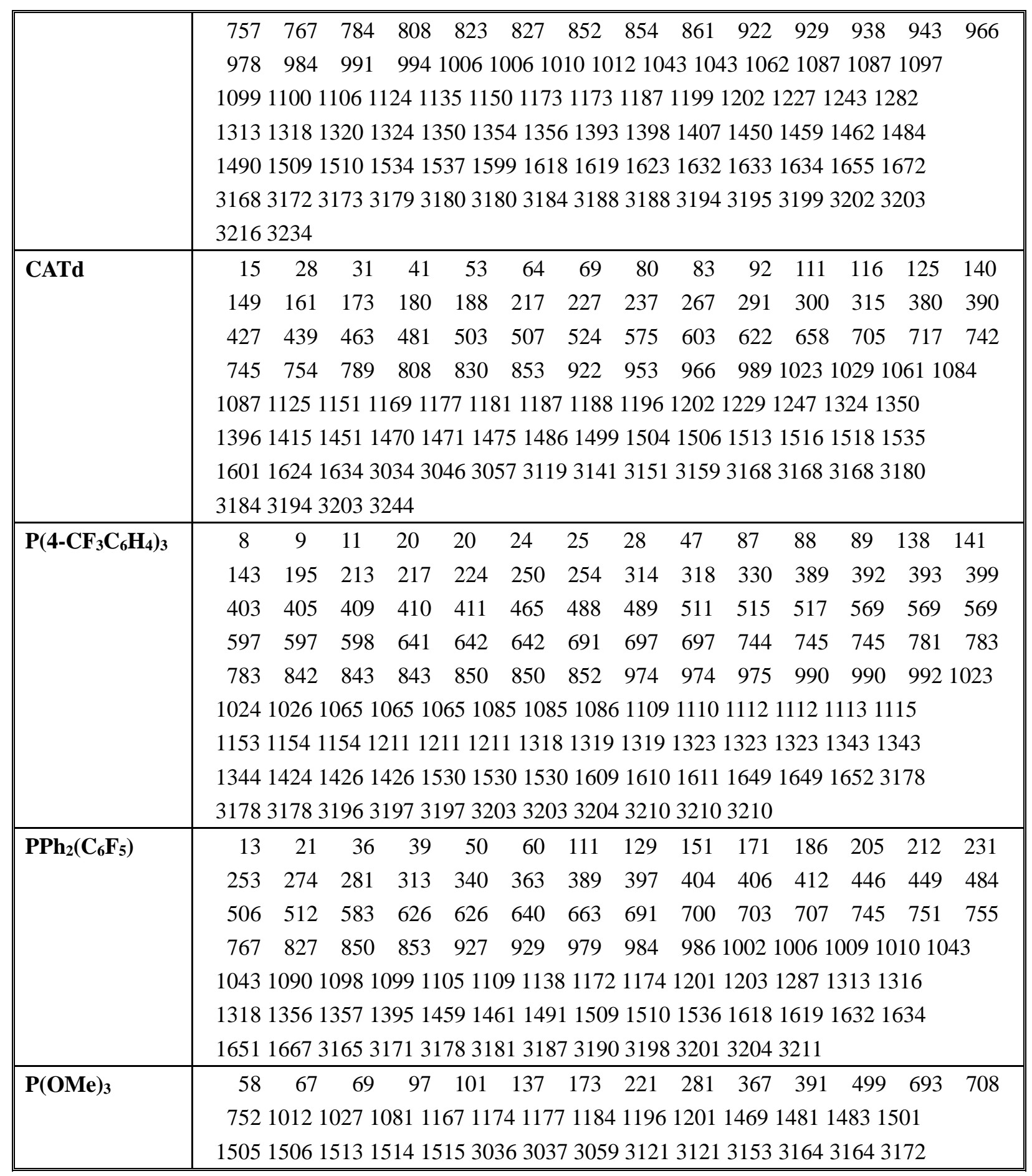

Table S1-5. Frequencies of some stationary points for the ligand exchange steps with the introduction of $\mathrm{CsCl}, \mathrm{LiF}, \mathrm{NaF}$ or KF, computed at IDSCRF (PCM)-B3LYP/DZVP level in DMA.

\begin{tabular}{|c|c|c|c|c|c|c|c|c|c|c|c|c|c|}
\hline Species & Freque & icies & $\left.\mathrm{sm}^{-1}\right)$ & & & & & & & & & & \\
\hline $\mathrm{CsCl}$ & 92 & & & & & & & & & & & & \\
\hline \multirow[t]{4}{*}{ LE-TS1Ba } & $-62 i$ & 13 & 29 & 42 & 46 & 48 & 80 & 92 & 111 & 129 & 136 & 153 & $\begin{array}{ll}160 & 179\end{array}$ \\
\hline & 201 & 208 & 235 & 246 & 267 & 274 & 312 & 315 & 375 & 388 & 431 & 459 & $478 \quad 485$ \\
\hline & 501 & 504 & 554 & 572 & 600 & 617 & 654 & 660 & 688 & 725 & 744 & 744 & $755 \quad 784$ \\
\hline & 807 & 813 & 826 & 838 & 843 & 854 & 900 & 912 & 925 & 927 & 968 & 983 & 9841003 \\
\hline
\end{tabular}




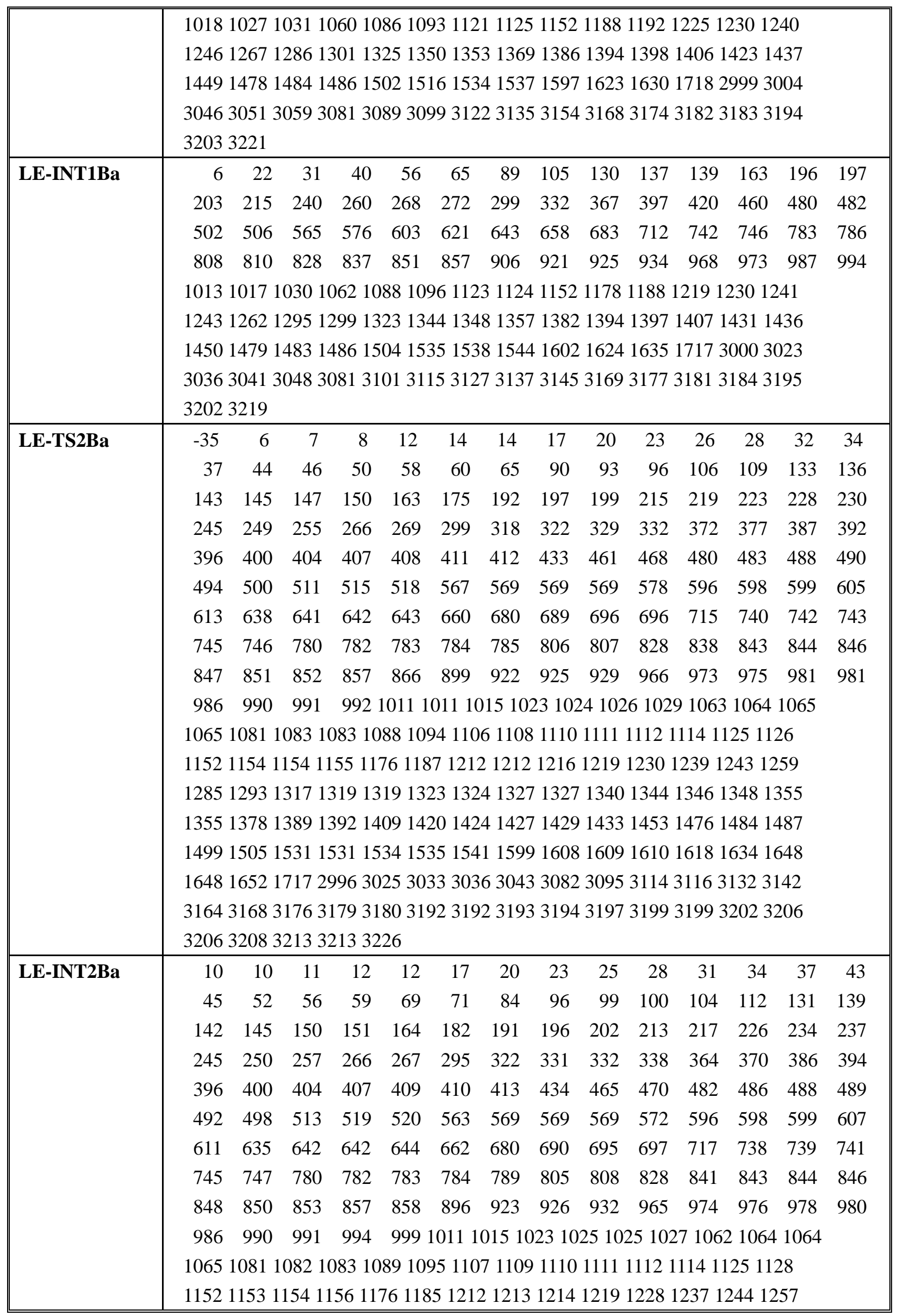




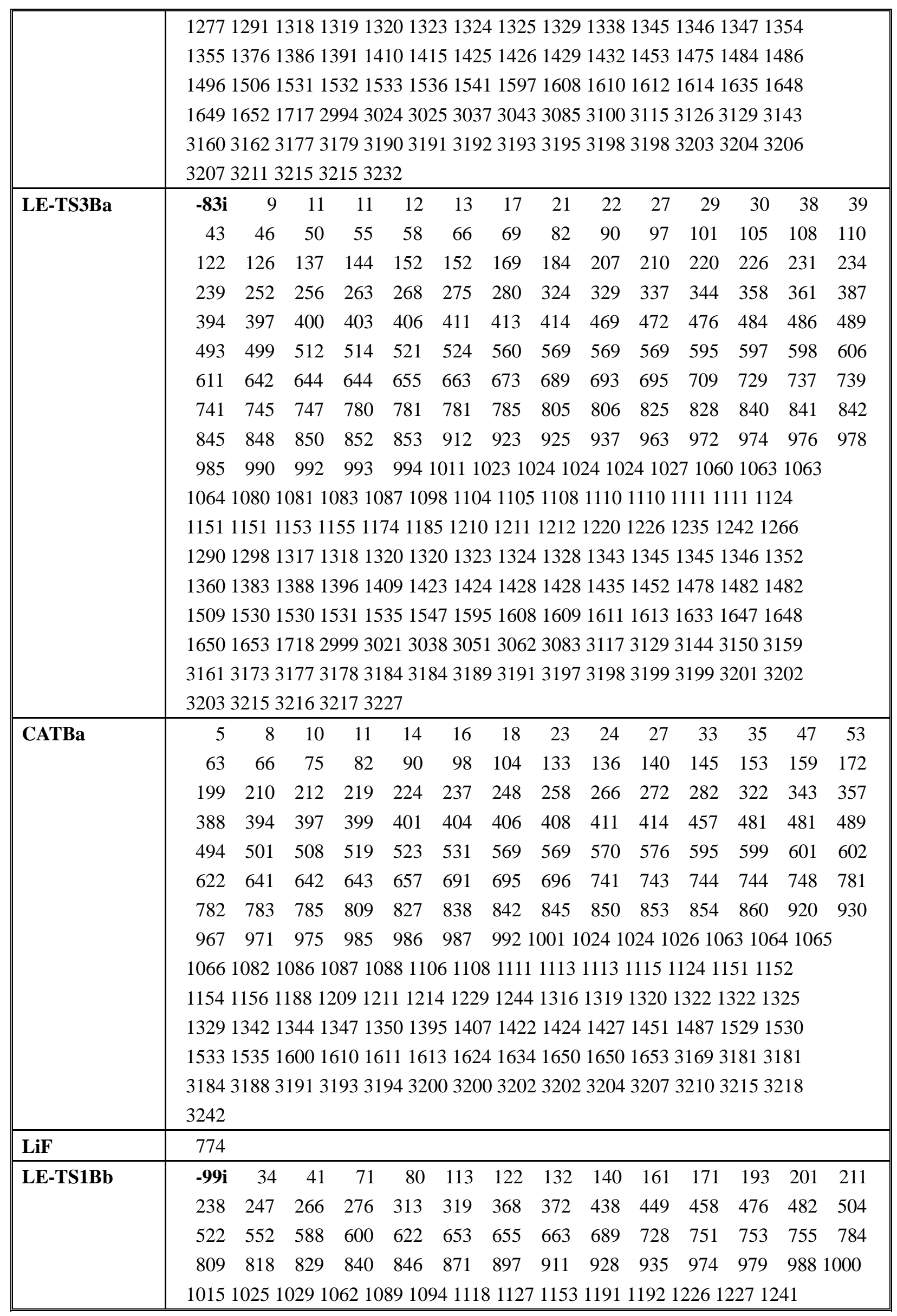




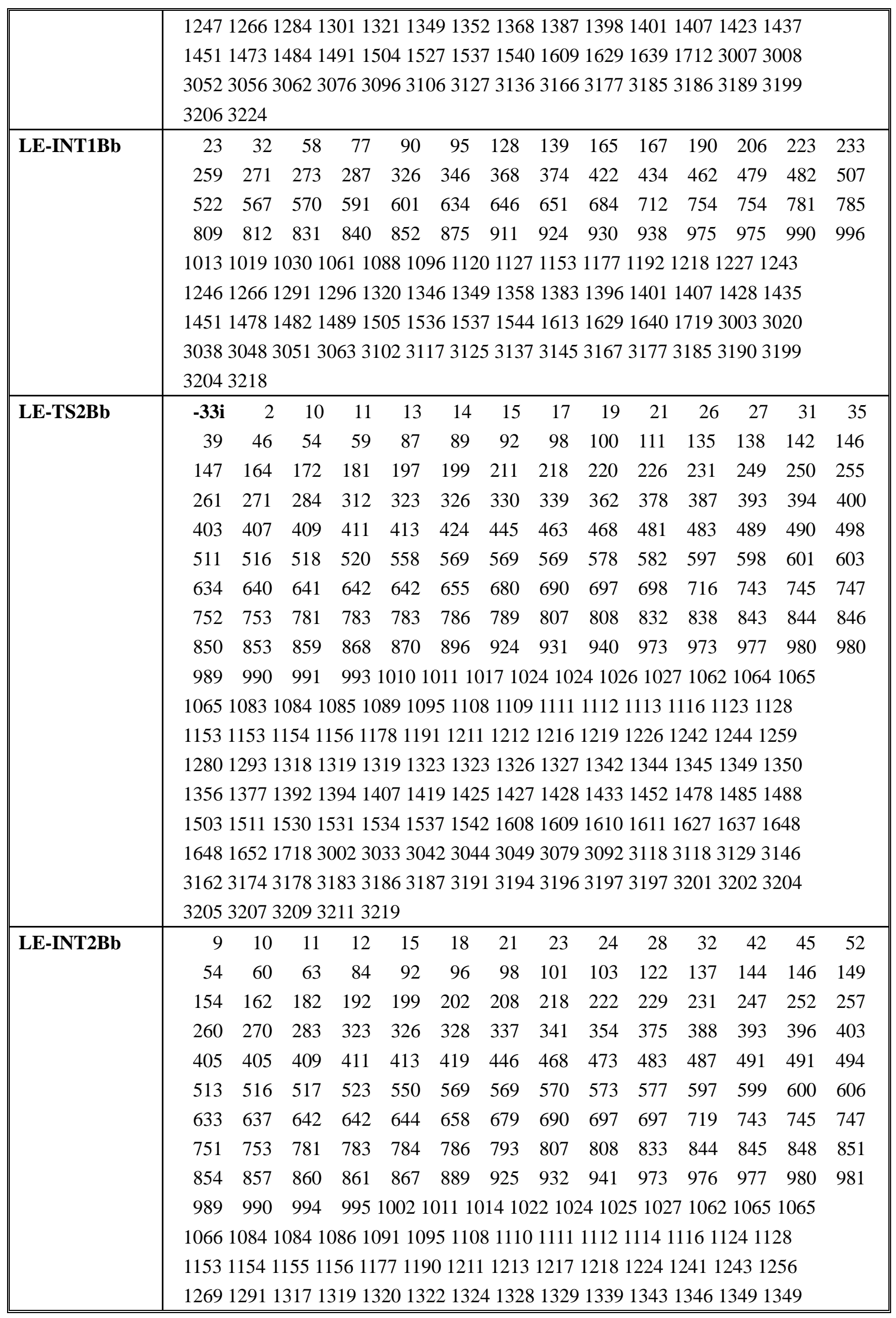




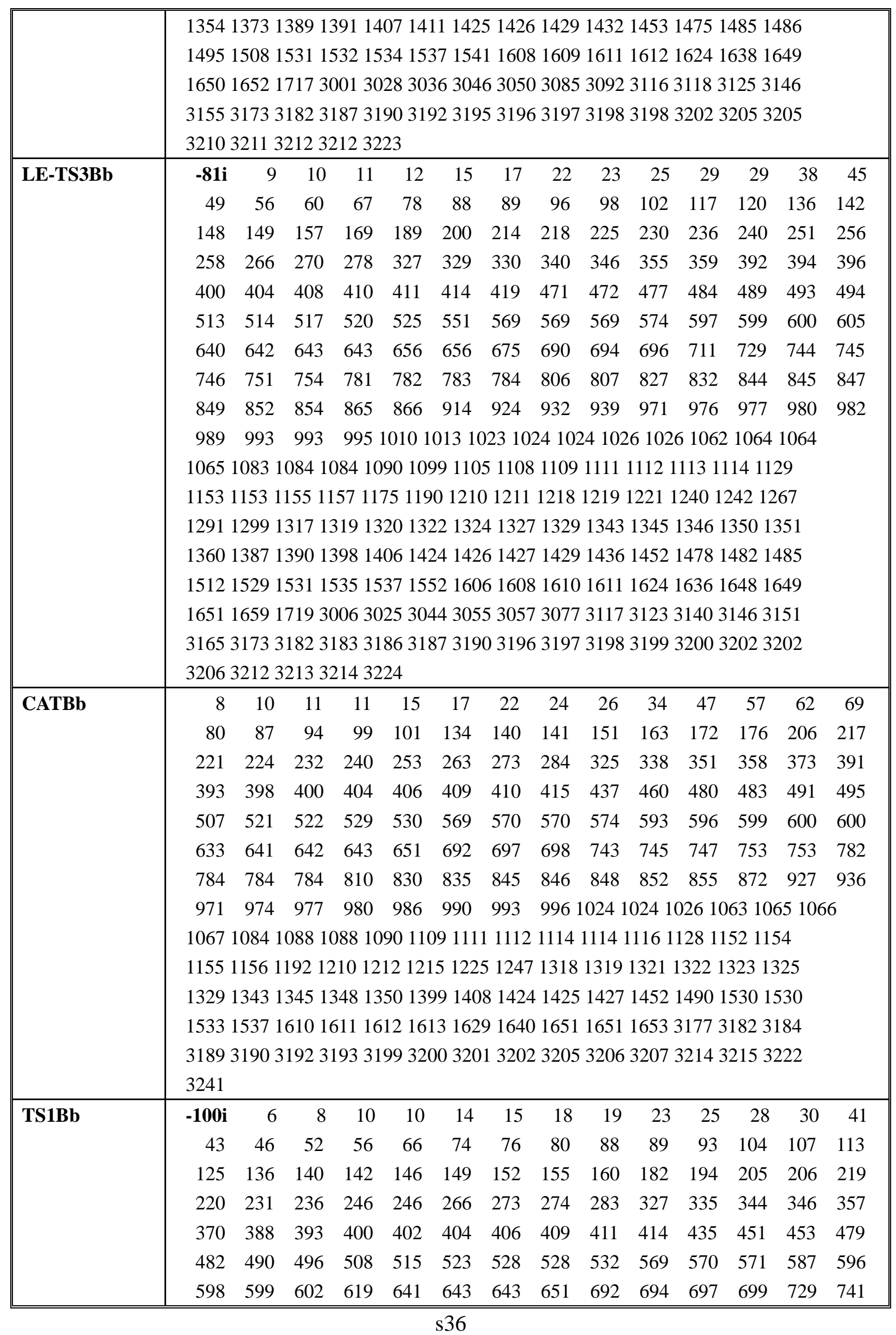




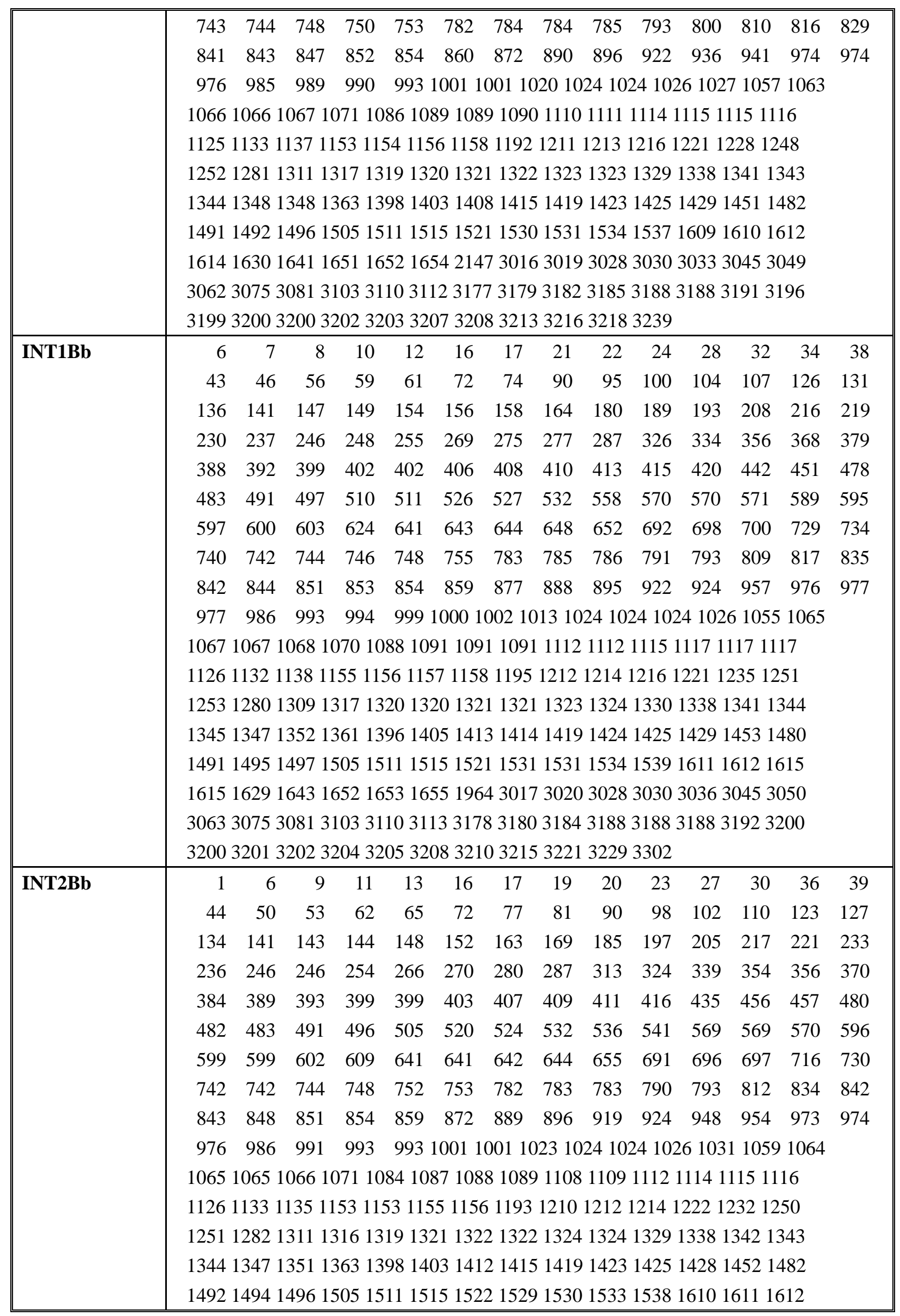




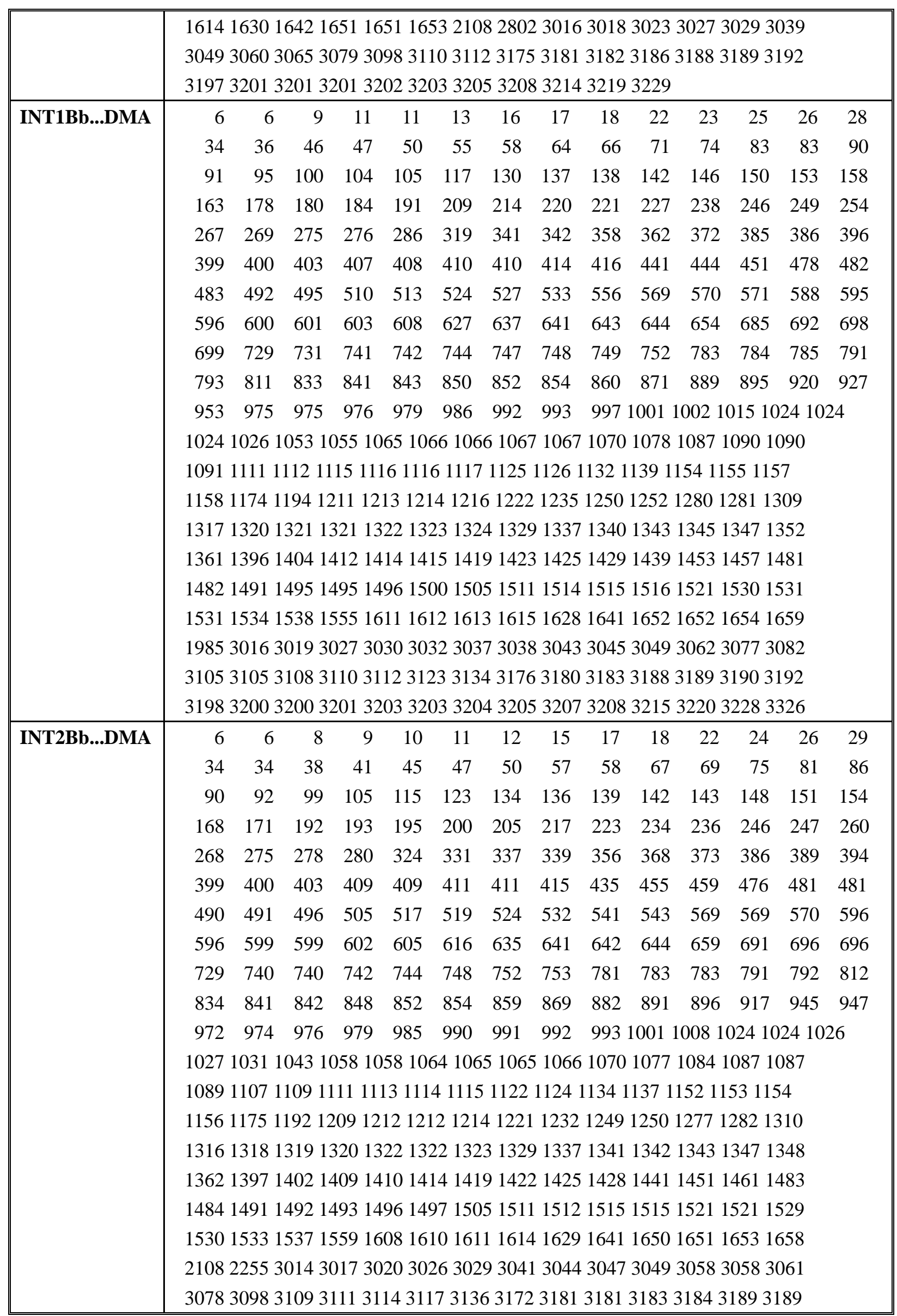




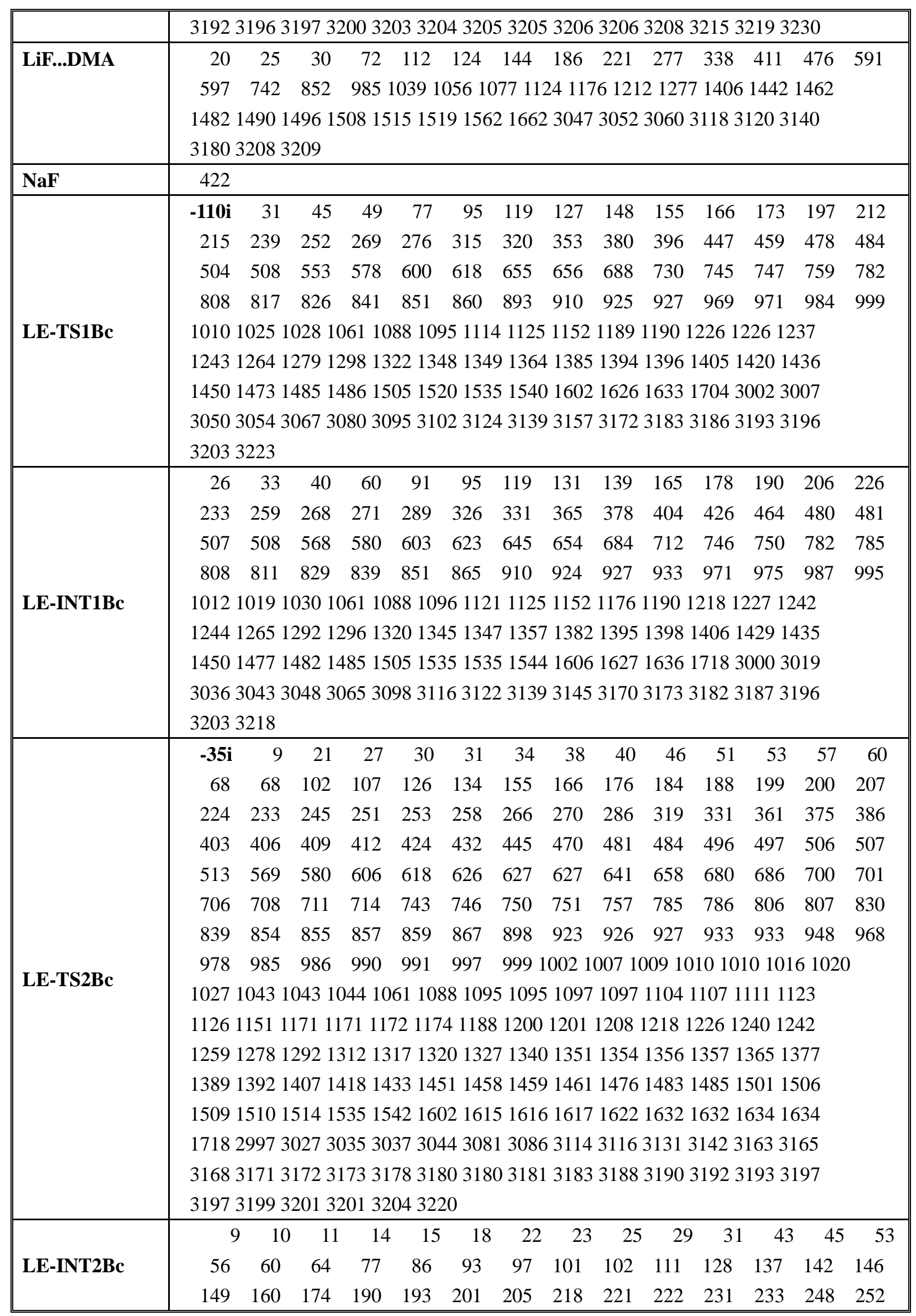




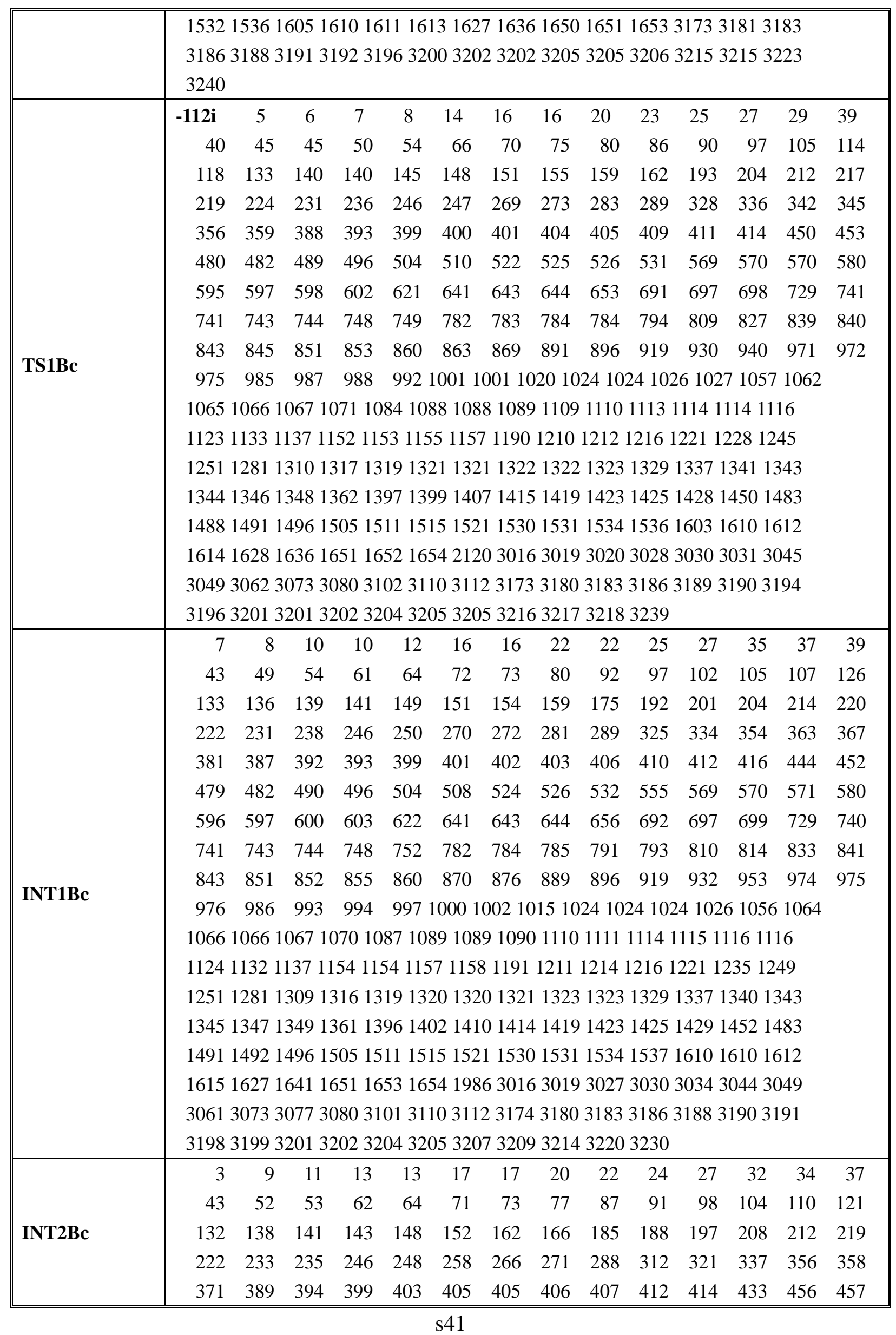




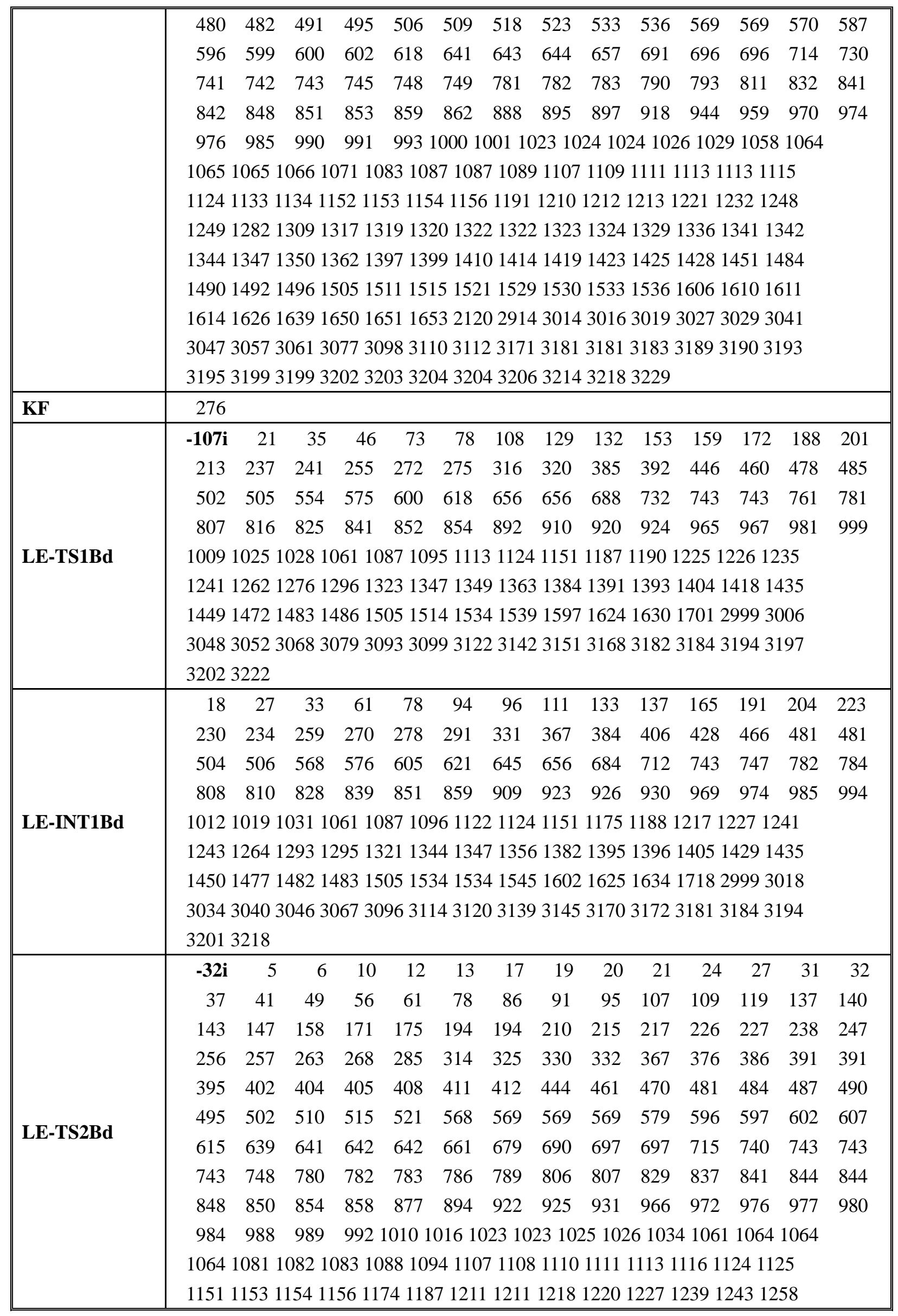




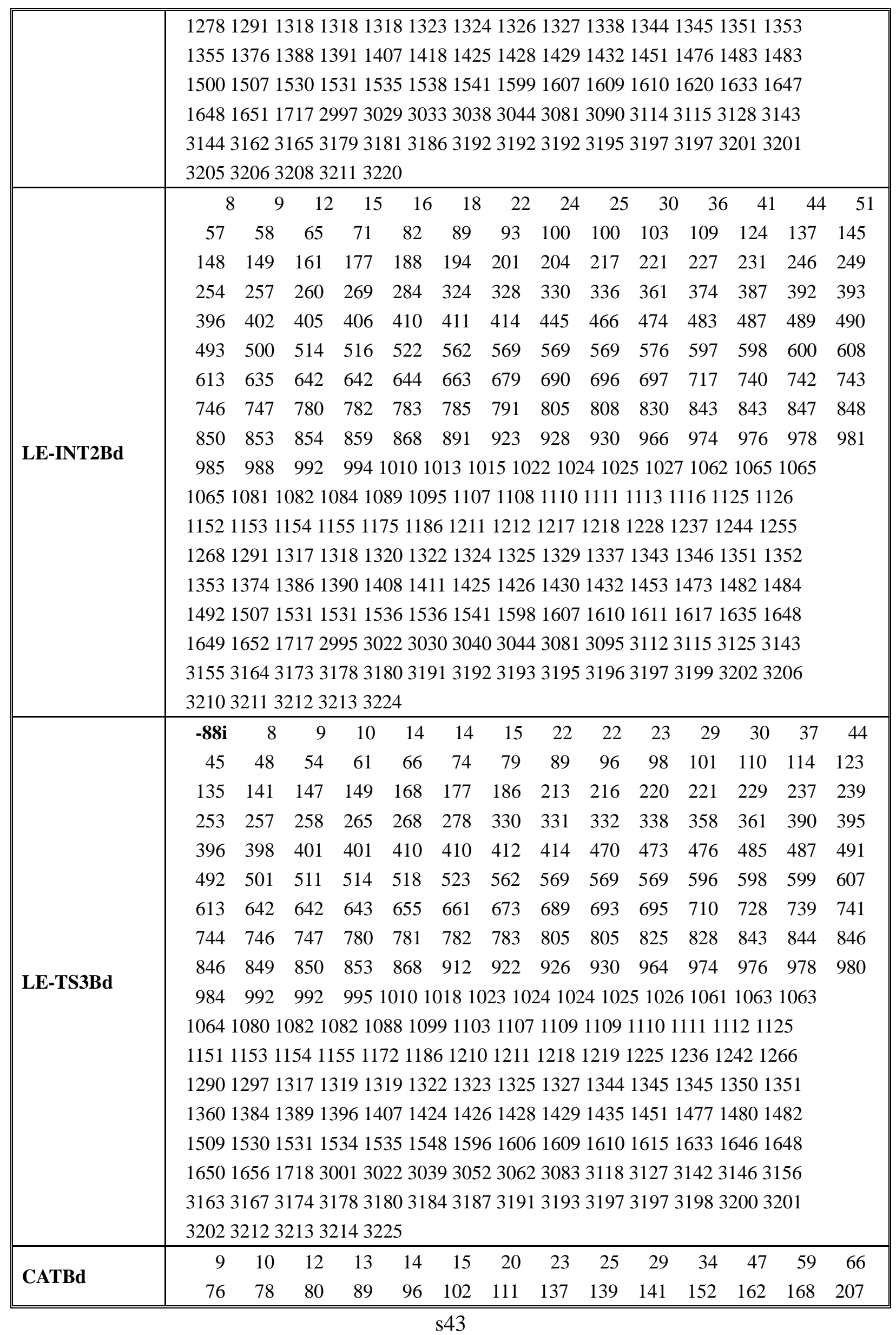




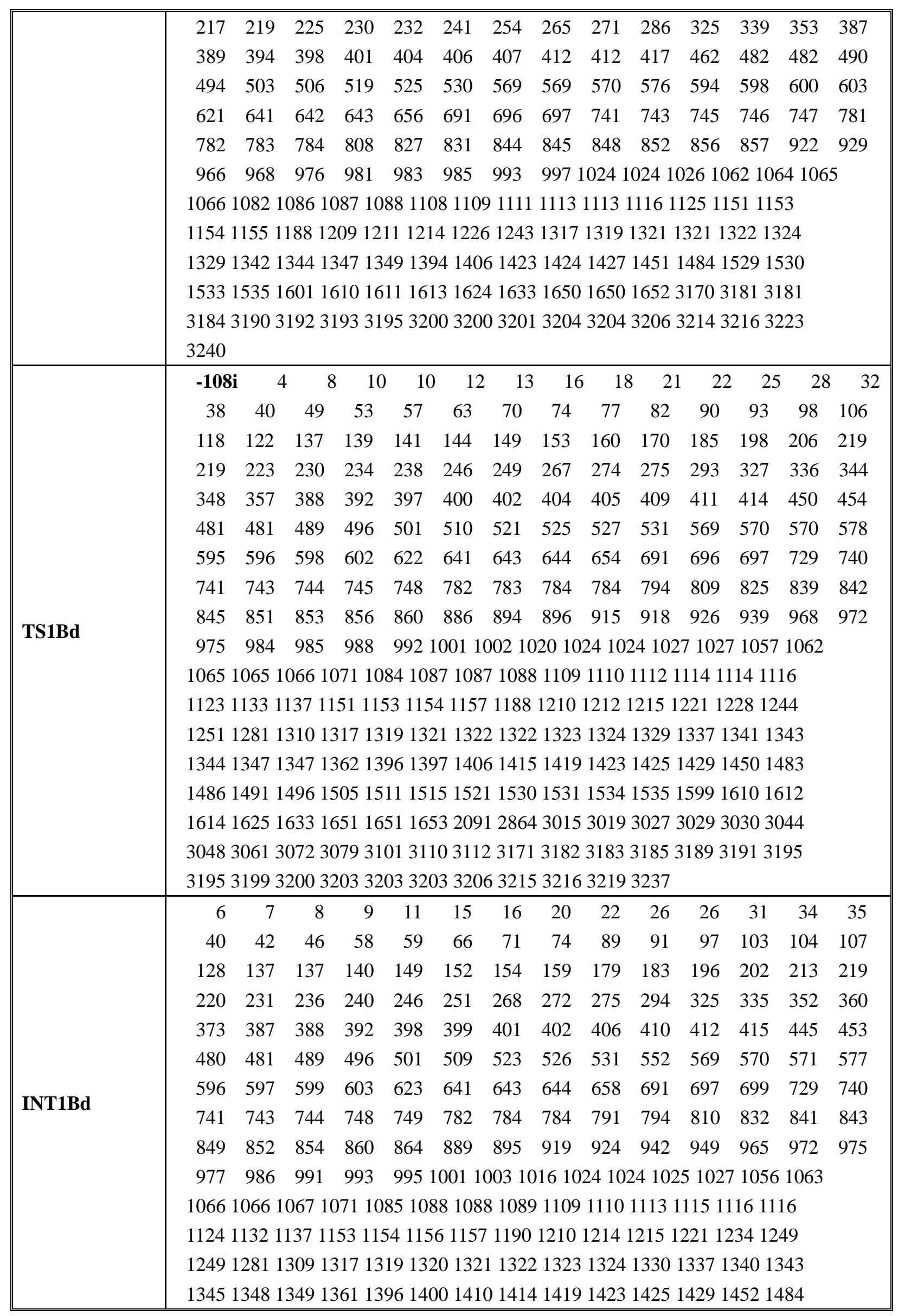




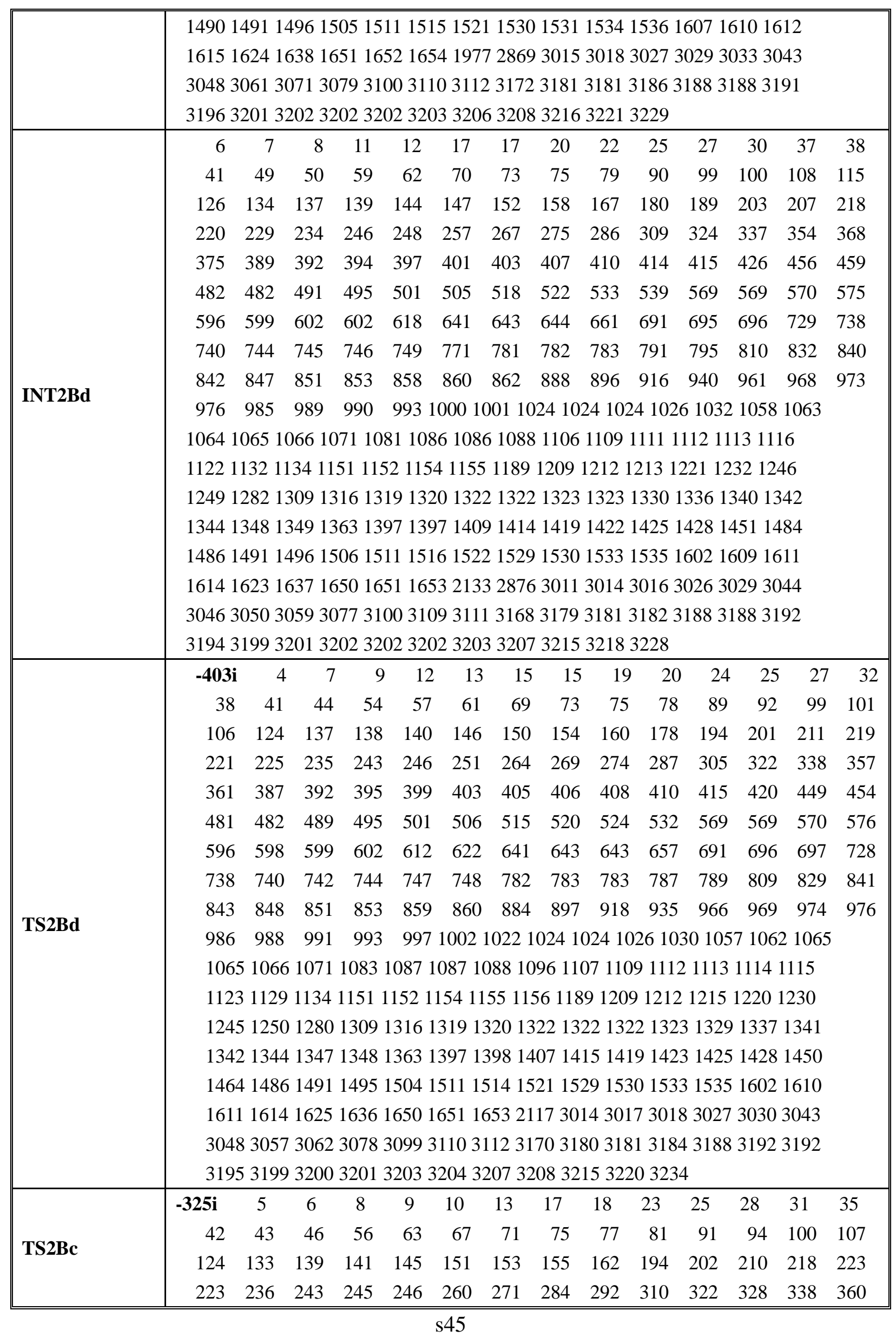




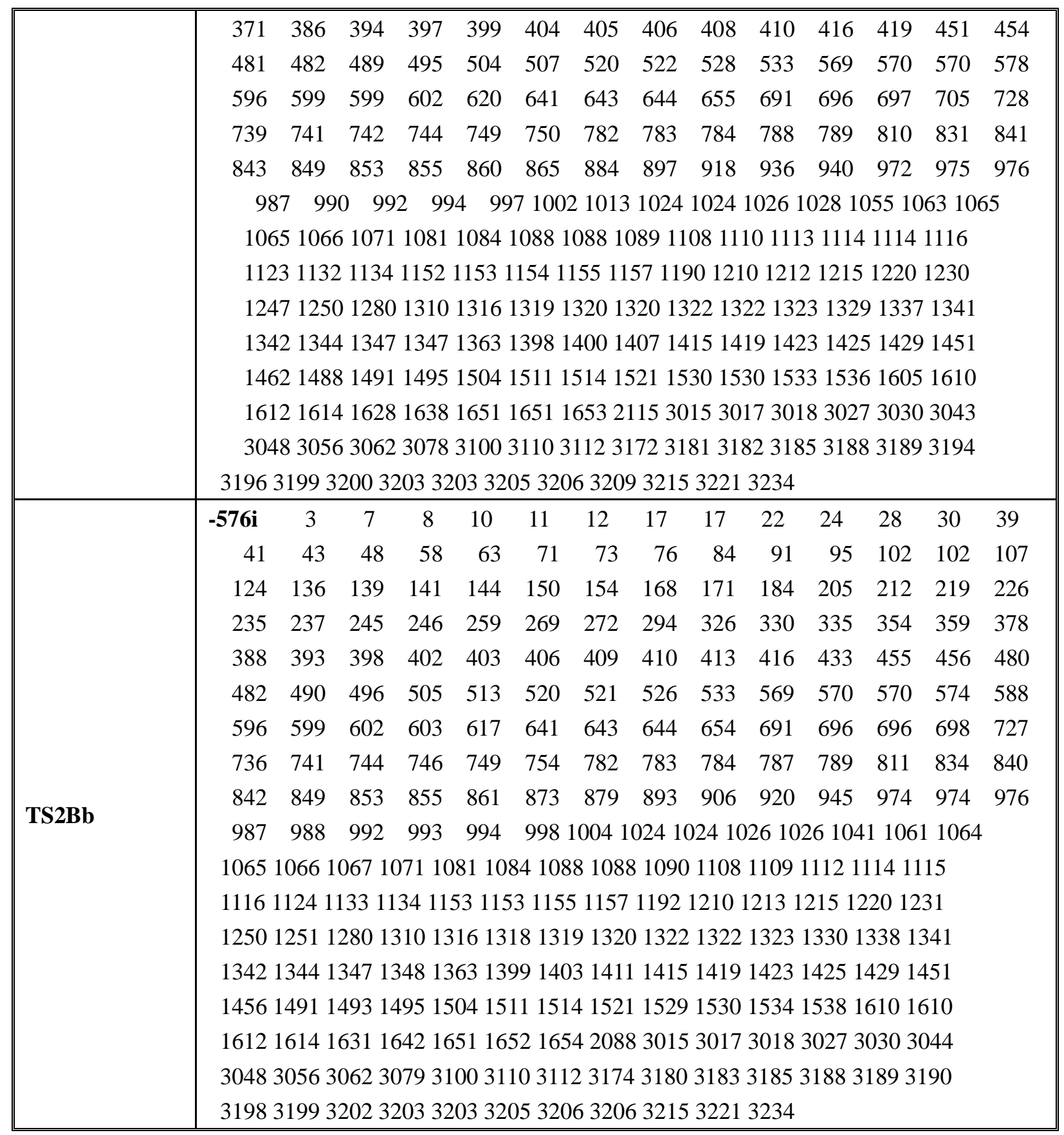

Table S1-6. Frequencies of some stationary points for the entire catalytic cycle when $\mathrm{Q}=2$-methyl-8-quinolinolato, computed at IDSCRF (PCM)-B3LYP/DZVP level in DMA.

\begin{tabular}{|c|c|}
\hline Species & Frequencies $\left(\mathrm{cm}^{-1}\right)$ \\
\hline \multirow[t]{7}{*}{ preCATm } & $\begin{array}{llllllllllllll}17 & 50 & 88 & 110 & 122 & 143 & 153 & 169 & 181 & 185 & 200 & 218 & 248 & 275\end{array}$ \\
\hline & $\begin{array}{llllllllllllll}319 & 321 & 330 & 341 & 373 & 424 & 432 & 443 & 470 & 477 & 502 & 517 & 521 & 565\end{array}$ \\
\hline & $\begin{array}{llllllllllllll}576 & 594 & 628 & 680 & 694 & 698 & 713 & 749 & 755 & 766 & 791 & 803 & 825 & 844\end{array}$ \\
\hline & 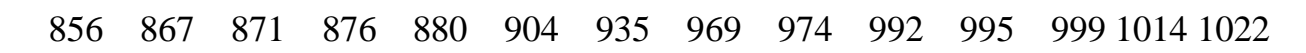 \\
\hline & 10251052108310951101112511671185119112061216123012481256 \\
\hline & 12611273131113351336134413561371137414001404141214191461 \\
\hline & 14741486148915031509151015351539154415471593163016463013 \\
\hline
\end{tabular}




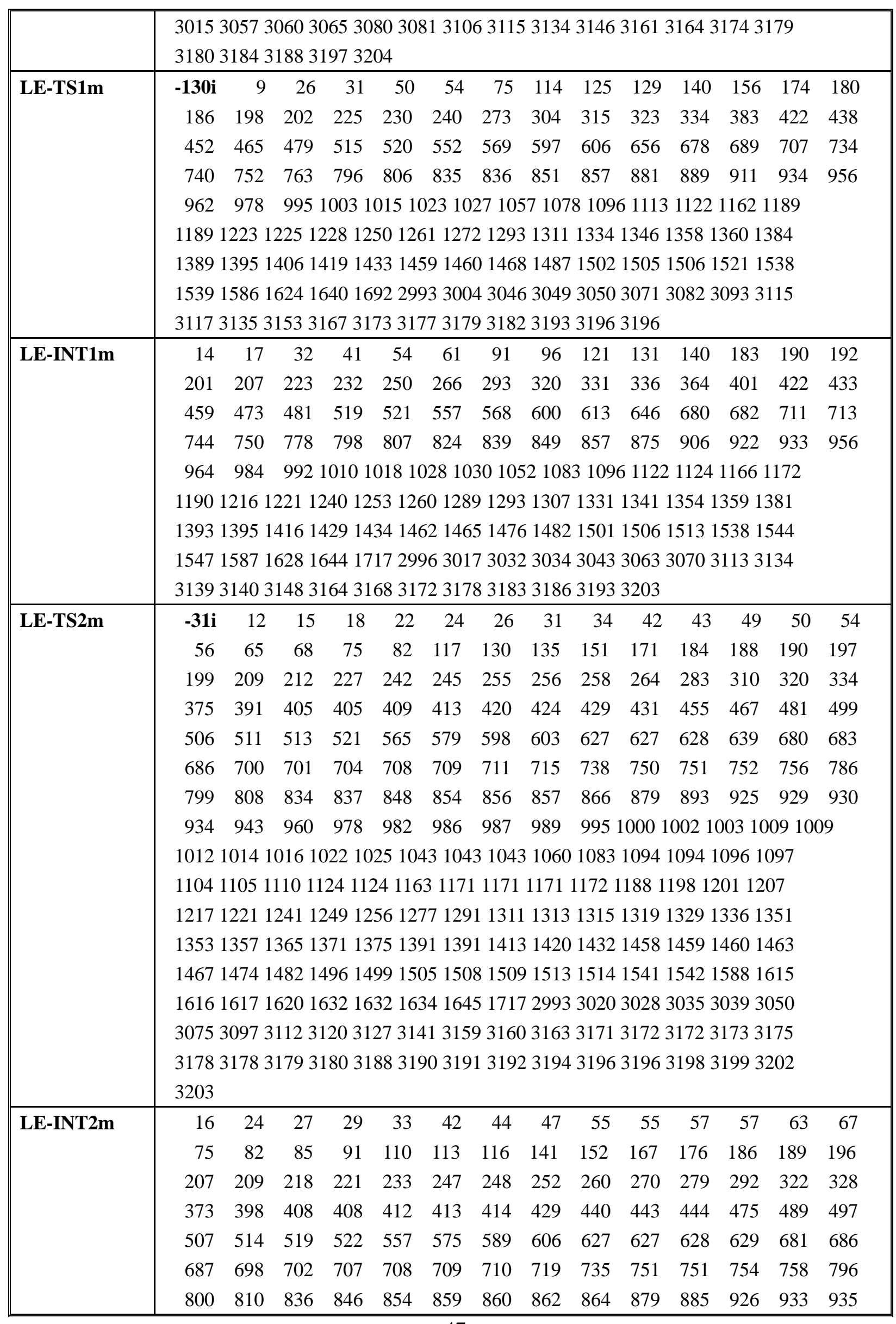




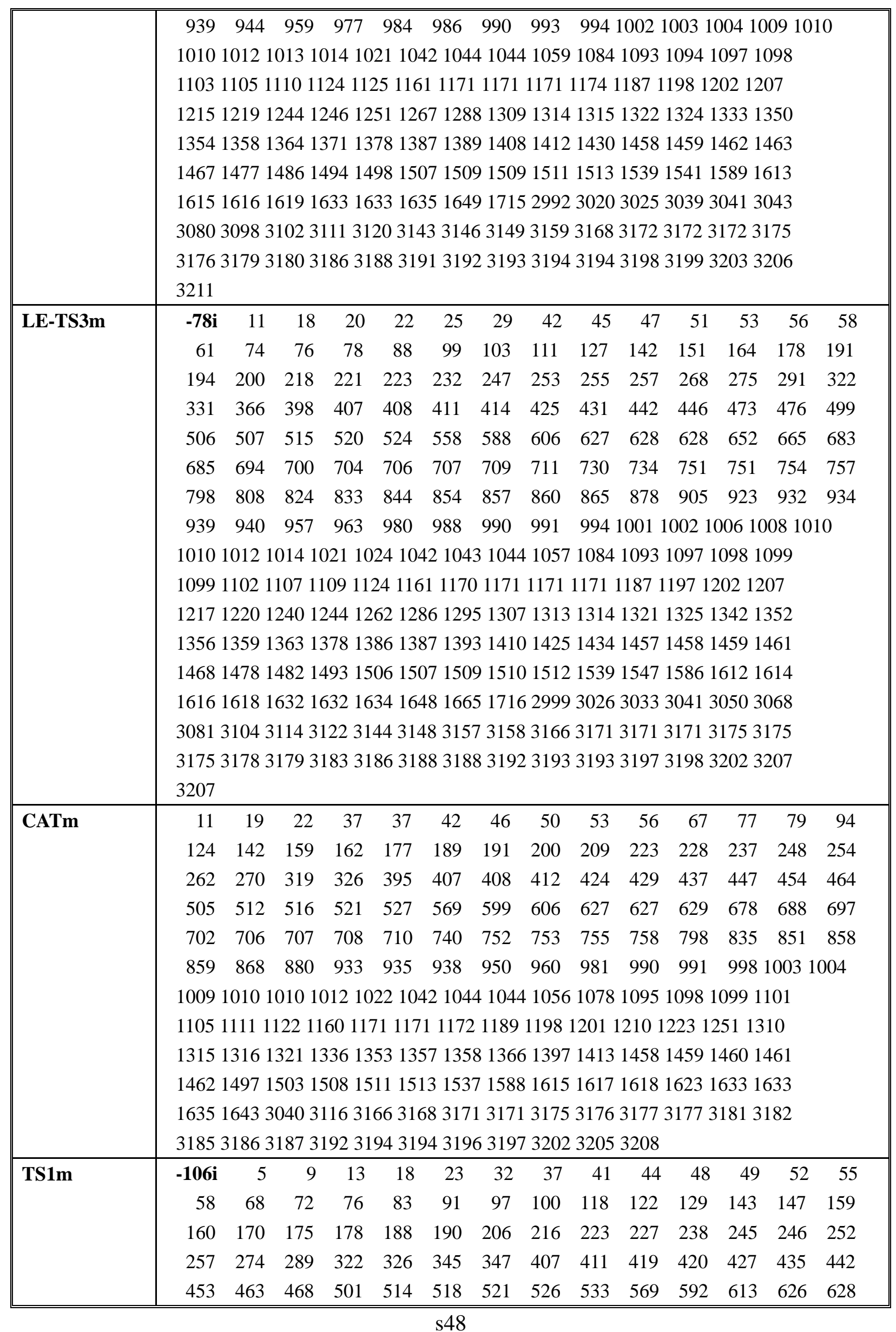




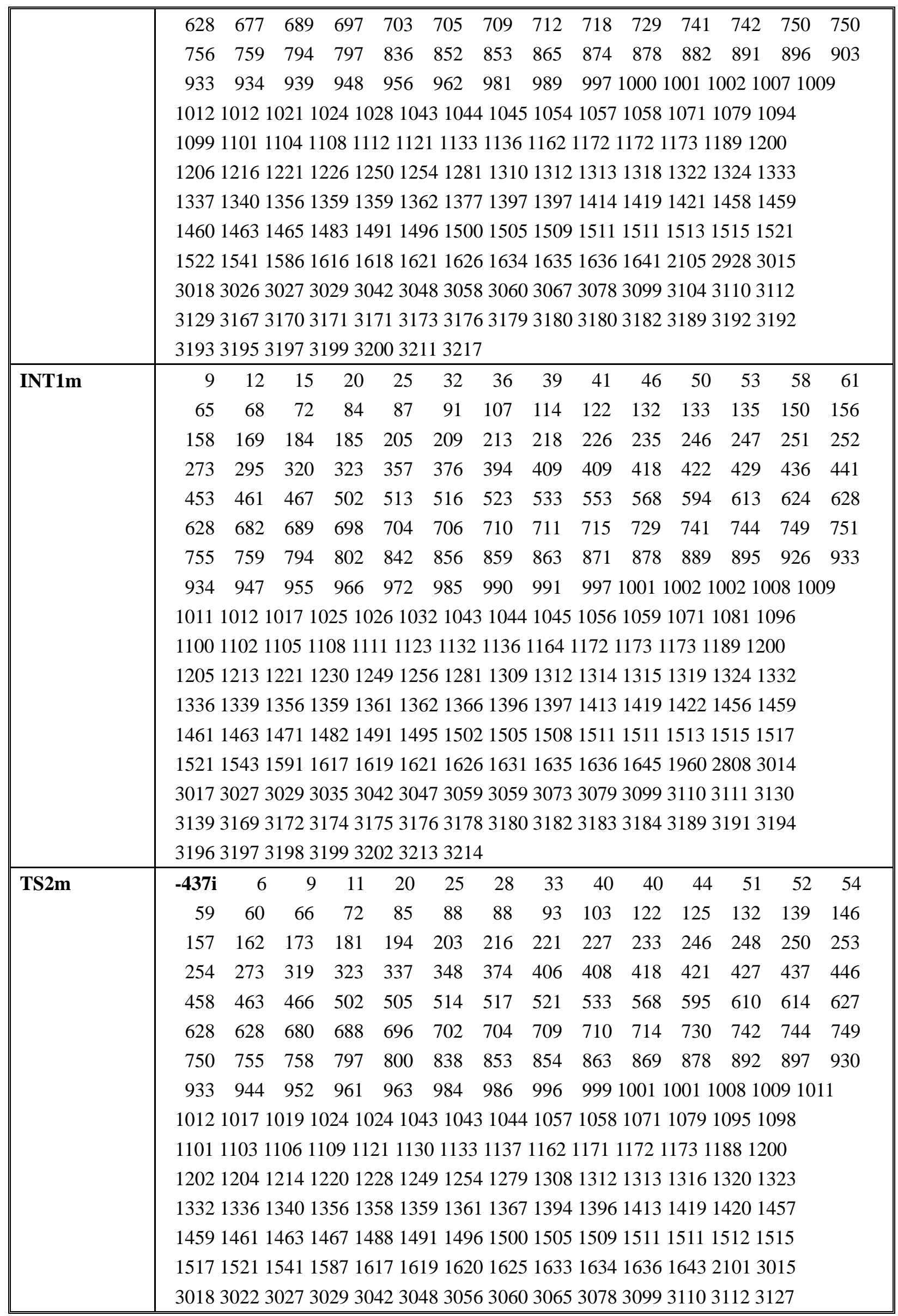




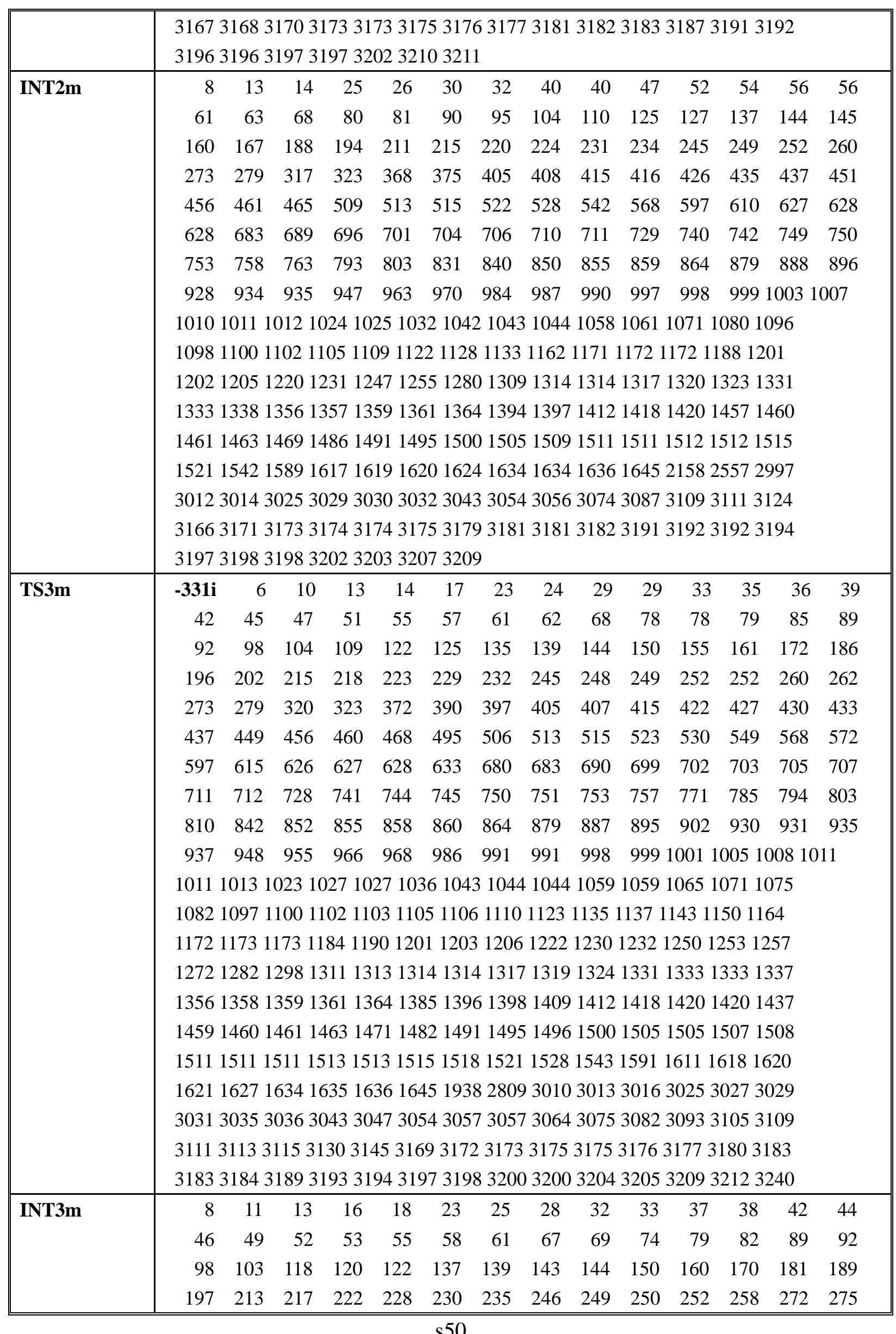




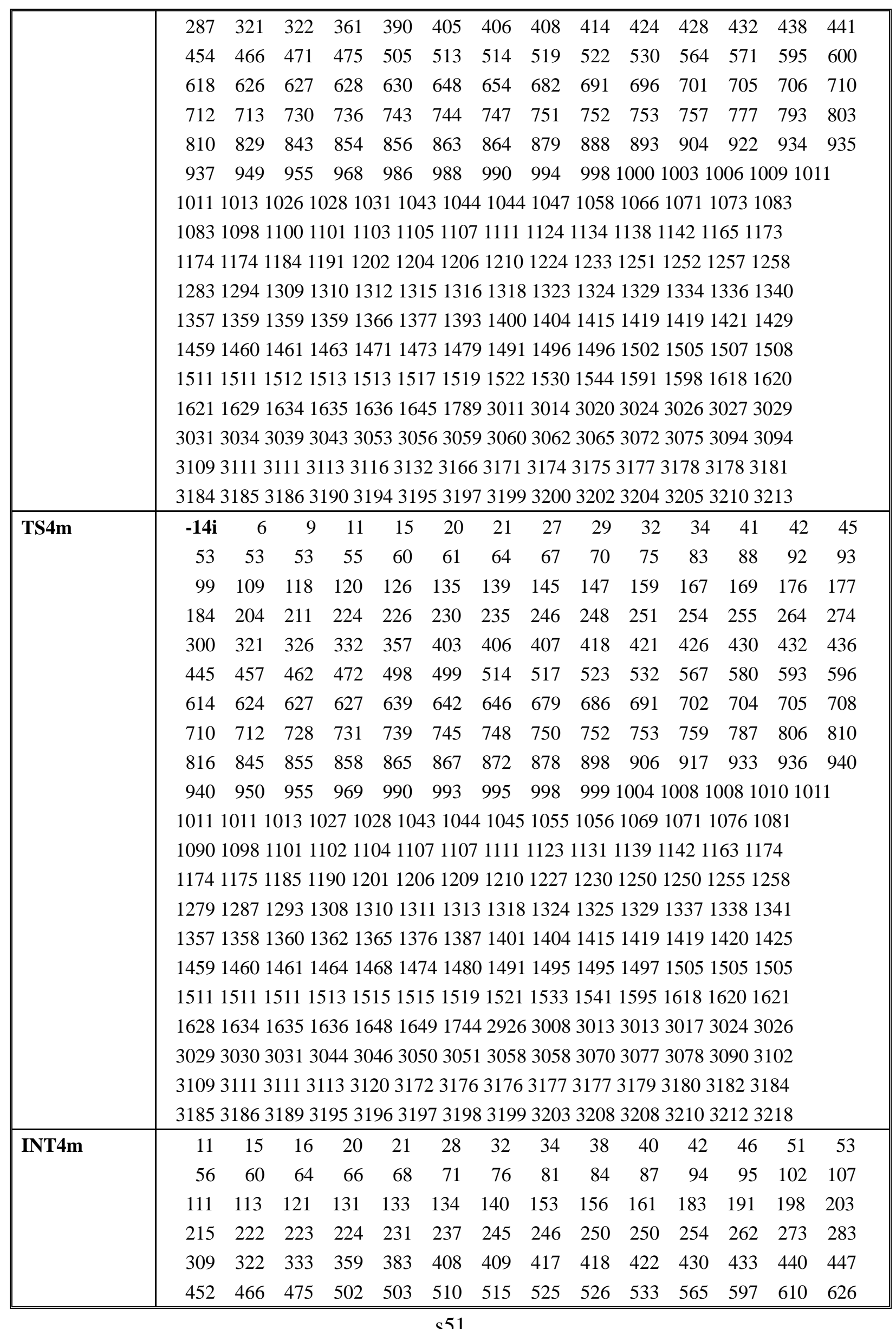




\begin{tabular}{|c|c|c|c|c|c|c|c|c|c|c|c|c|c|c|}
\hline & 628 & 628 & 642 & 687 & 687 & 694 & 698 & 706 & 707 & 711 & 713 & 721 & 723 & 737 \\
\hline & 741 & 744 & 747 & 750 & 753 & 759 & 786 & 793 & 804 & 810 & 811 & 840 & 853 & 854 \\
\hline & 855 & 858 & 866 & 875 & 883 & 897 & 898 & 910 & 921 & 929 & 932 & 941 & 949 & 955 \\
\hline & 961 & 962 & 972 & 986 & 988 & 994 & 995 & 9991 & 0001 & 00710 & 00710 & 1010 & 12101 & \\
\hline & \multirow{11}{*}{\multicolumn{14}{|c|}{$\begin{array}{l}10131023102710301041104210431044105510591061107110751086 \\
10921095109811001101110311081121112511441145115411641171 \\
11721172117311841188120012041207122212311244125512551262 \\
12781284130013111314131613171320132213241325133313341336 \\
13401354135813591371137813891392141014161419142014231430 \\
14571459146214621475148314841490149414961499150315051509 \\
15101511151115121514151415171521152515441586161616191620 \\
16231629163316341636164717112265297630003007300830133024 \\
30283029303230373041305530603065306630663068307130743088 \\
31083110311331173118312831473160316431703171317331733177 \\
31783181318131853190319231943196319831993200320932133215 \\
\end{array}$}} \\
\hline & & & & & & & & & & & & & & \\
\hline & & & & & & & & & & & & & & \\
\hline & & & & & & & & & & & & & & \\
\hline & & & & & & & & & & & & & & \\
\hline & & & & & & & & & & & & & & \\
\hline & & & & & & & & & & & & & & \\
\hline & & & & & & & & & & & & & & \\
\hline & & & & & & & & & & & & & & \\
\hline & & & & & & & & & & & & & & \\
\hline & & & & & & & & & & & & & & \\
\hline \multirow[t]{21}{*}{ TS5m } & $-930 i$ & 11 & 14 & 16 & 20 & 26 & 27 & 28 & 36 & 37 & 40 & 43 & 48 & 49 \\
\hline & 52 & 55 & 55 & 59 & 68 & 72 & 73 & 80 & 82 & 91 & 96 & 98 & 103 & 110 \\
\hline & 113 & 119 & 124 & 130 & 136 & 140 & 149 & 157 & 163 & 169 & 176 & 182 & 189 & 193 \\
\hline & 202 & 206 & 219 & 224 & 228 & 233 & 240 & 243 & 246 & 250 & 251 & 255 & 259 & 272 \\
\hline & 308 & 319 & 323 & 354 & 376 & 392 & 407 & 410 & 417 & 418 & 423 & 428 & 432 & 443 \\
\hline & 446 & 451 & 465 & 476 & 497 & 505 & 508 & 515 & 515 & 522 & 528 & 568 & 595 & 608 \\
\hline & 627 & 627 & 629 & 664 & 682 & 687 & 693 & 698 & 707 & 708 & 710 & 714 & 726 & 736 \\
\hline & 737 & 741 & 744 & 750 & 752 & 754 & 759 & 769 & 786 & 801 & 811 & 839 & 853 & 855 \\
\hline & 858 & 858 & 865 & 878 & 878 & 899 & 908 & 933 & 934 & 937 & 940 & 948 & 954 & 961 \\
\hline & 963 & 967 & 986 & 990 & 991 & 993 & 999 & 10011 & 00510 & 0610 & 11101 & 11101 & 41016 & \\
\hline & 1024 & 024 & 0271 & 33210 & 4210 & 43104 & 4105 & 41055 & 1058 & 10701 & 10731 & 0751 & 080 & \\
\hline & 1083 & 094 & 0971 & 99811 & 01110 & 2110 & 81122 & 21126 & 1145 & 1501 & 16311 & 16311 & & \\
\hline & 11721 & 1721 & 18411 & 8911 & 96120 & 0120 & 21206 & 51223 & 1228 & 12501 & 25512 & 25512 & 266 & \\
\hline & 12801 & 282 & 2911 & 30113 & 11131 & 14131 & 7131 & 81322 & 1324 & 13301 & 13321 & 3331 & 335 & \\
\hline & 1335 & 345 & 3541 & 35713 & 58136 & 65138 & 1138 & 91395 & 1411 & 14181 & 14191 & 4201 & 424 & \\
\hline & 14301 & 457 & 4601 & 46114 & 6214 & 58146 & 8147 & 21492 & 1496 & 14971 & 14991 & 5041 & 507 & \\
\hline & 1508 & 510 & 5111 & 51115 & 11151 & 2151 & 61518 & 81523 & 1525 & 15411 & 15841 & 58916 & 616 & \\
\hline & 1618 & 619 & 6241 & 63316 & 3416 & 35164 & 4172 & 02991 & 3008 & 30143 & 30253 & 0283 & 028 & \\
\hline & 30293 & $032 ?$ & 0323 & 04030 & 4130 & 55305 & 5306 & 33065 & 3066 & 30753 & 30823 & 0893 & 101 & \\
\hline & 31083 & 1103 & 1133 & 1731 & 18312 & 4312 & 53167 & 73170 & 3173 & 31733 & 31753 & 17731 & 178 & \\
\hline & 31813 & 1813 & 1863 & 18631 & 92315 & 94319 & 4319 & 63197 & 3199 & 32003 & 32073 & 2173 & 218 & \\
\hline \multirow[t]{8}{*}{ INT5m } & 6 & 12 & 13 & 17 & 18 & 21 & 21 & 23 & 29 & 30 & 34 & 37 & 38 & 43 \\
\hline & 45 & 48 & 49 & 56 & 59 & 60 & 62 & 67 & 76 & 79 & 80 & 87 & 100 & 105 \\
\hline & 107 & 115 & 119 & 123 & 127 & 150 & 151 & 157 & 159 & 162 & 165 & 172 & 190 & 192 \\
\hline & 193 & 200 & 214 & 216 & 227 & 231 & 231 & 244 & 247 & 248 & 253 & 257 & 263 & 272 \\
\hline & 273 & 322 & 330 & 333 & 370 & 404 & 411 & 416 & 420 & 423 & 425 & 429 & 442 & 450 \\
\hline & 451 & 459 & 464 & 474 & 481 & 493 & 511 & 518 & 519 & 522 & 568 & 595 & 617 & 618 \\
\hline & 624 & 626 & 643 & 679 & 684 & 698 & 703 & 711 & 712 & 715 & 718 & 727 & 730 & 737 \\
\hline & 744 & 744 & 746 & 755 & 756 & 757 & 772 & 791 & 800 & 811 & 840 & 842 & 846 & 854 \\
\hline
\end{tabular}




\begin{tabular}{|c|c|}
\hline & 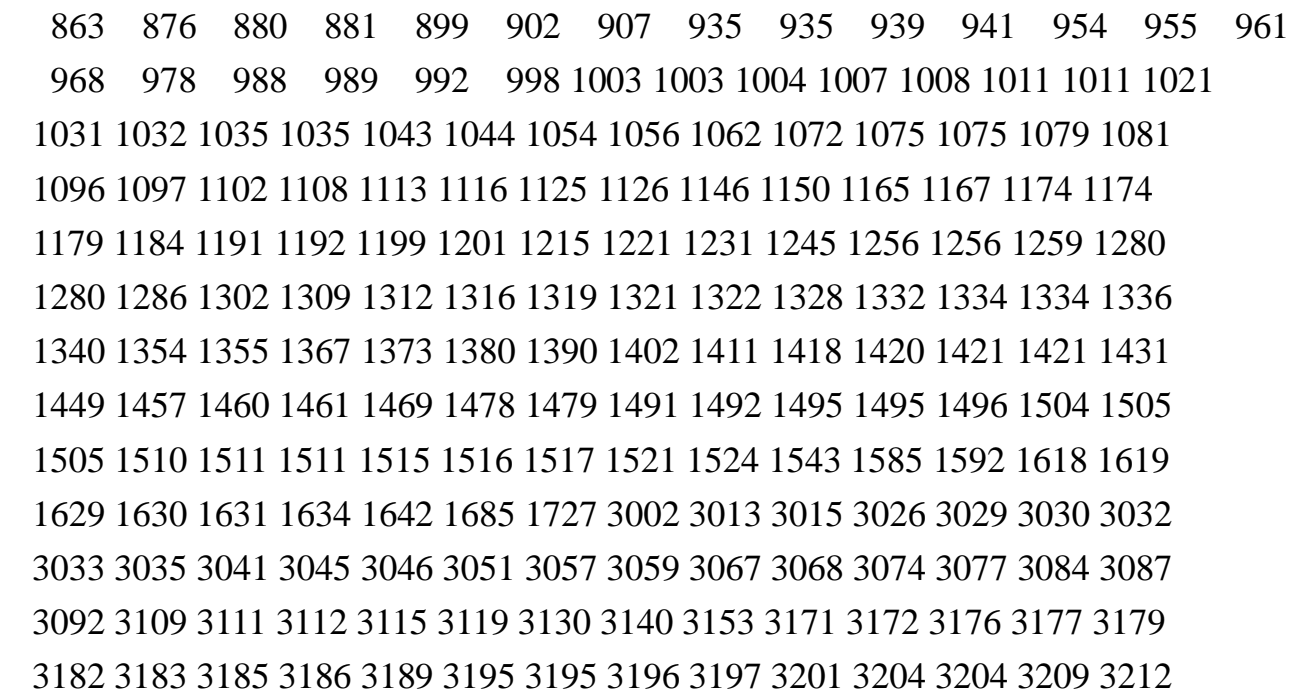 \\
\hline
\end{tabular}

Table S2-1. The electronic energies (Eele /a.u.), total energies $\left(E_{\text {tot }} /\right.$ a.u. $=E_{\text {ele }} /$ a.u. $\left.+Z P E / a . u.\right)$, enthalpy (H/a.u.), Gibbs free energies in gas $\left(\mathrm{G}_{1} /\right.$ a.u. $)$ and Gibbs free energies in solution $\left(\mathrm{G}_{2} /\right.$ a.u. $)$ for the ligand exchange process, computed at IDSCRF(PCM)-B3LYP/DZVP level in DMA at $353 \mathrm{~K}$.

\begin{tabular}{|l|l|l|l|l|l||}
\hline \hline Species & $\mathrm{E}_{\text {ele }} /$ a.u. & $\mathrm{E}_{\text {tot }} /$ a.u. & H/a.u. & $\mathrm{G}_{1} /$ a.u. & $\mathrm{G}_{2} /$ a.u. \\
\hline preCAT & -5476.41160 & -5476.09942 & -5476.07488 & -5476.15552 & -5476.14153 \\
\hline LE-TS1 & -5596.50213 & -5596.18970 & -5596.16011 & -5596.25684 & -5596.24264 \\
\hline LE-INT1 & -5596.53964 & -5596.22656 & -5596.19615 & -5596.29628 & -5596.28191 \\
\hline LE-TS2 & -7644.22216 & -7643.62388 & -7643.55488 & -7643.75325 & -7643.73932 \\
\hline LE-INT2 & -7644.22395 & -7643.62512 & -7643.55545 & -7643.75402 & -7643.74039 \\
\hline LE-TS3 & -7644.21336 & -7643.61535 & -7643.54580 & -7643.74596 & -7643.73249 \\
\hline CAT & -7332.17606 & -7331.75845 & -7331.70096 & -7331.87267 & -7331.85836 \\
\hline P(4-CF $\left.\mathbf{C}_{\mathbf{6}} \mathbf{H}_{\mathbf{4}}\right)_{3} \mathbf{3}$ & -2047.68904 & -2047.40401 & -2047.36611 & -2047.48957 & -2047.47559 \\
\hline cod & -312.05862 & -311.87836 & -311.86691 & -311.91746 & -311.90368 \\
\hline CsF & -120.11764 & -120.11725 & -120.11256 & -120.14720 & -120.13862 \\
\hline LE-TS1a & -5596.49887 & -5596.18707 & -5596.15710 & -5596.25561 & -5596.24165 \\
\hline LE-TS1b & -7524.06808 & -7523.46990 & -7523.40646 & -7523.58517 & -7523.57155 \\
\hline CATa & -7332.17398 & -7331.75653 & -7331.69890 & -7331.87158 & -7331.85740 \\
\hline
\end{tabular}

Table S2-2. The electronic energies (Eele/a.u.), total energies ( $E_{\text {tot }} /$ a.u. $=E_{\text {ele }} /$ a.u. $\left.+Z P E / a . u.\right)$, enthalpy (H/a.u.), Gibbs free energies in gas $\left(\mathrm{G}_{1} / \mathrm{a}\right.$.u. $)$ and Gibbs free energies in solution $\left(\mathrm{G}_{2} /\right.$ a.u. $)$ for the entire catalytic cycle, computed at IDSCRF(PCM)-B3LYP/DZVP level in DMA at 353K.

\begin{tabular}{|l|l|l|l|l|l||}
\hline \hline Species & E ele $/$ a.u. & $\mathrm{E}_{\text {tot }} /$ a.u. & H/a.u. & $\mathrm{G}_{1} /$ a.u. & $\mathrm{G}_{2} /$ a.u. \\
\hline 1a & -313.25262 & -313.05418 & -313.03920 & -313.09931 & -313.08660 \\
\hline 2a & -424.47437 & -424.29377 & -424.27848 & -424.34050 & -424.32755 \\
\hline TS1 & -7645.39968 & -7644.78352 & -7644.71051 & -7644.92224 & -7644.90894 \\
\hline INT1 & -7645.42688 & -7644.80937 & -7644.73629 & -7644.94351 & -7644.93031 \\
\hline
\end{tabular}




\begin{tabular}{|l|l|l|l|l|l||}
\hline TS2 & -7645.40676 & -7644.79401 & -7644.72112 & -7644.93138 & -7644.91801 \\
\hline INT2 & -7645.42406 & -7644.80711 & -7644.73363 & -7644.94449 & -7644.93135 \\
\hline TS3 & -8069.87049 & -8069.07279 & -8068.98413 & -8069.23339 & -8069.22066 \\
\hline INT3 & -8069.87361 & -8069.07461 & -8068.98564 & -8069.23471 & -8069.22190 \\
\hline TS4 & -8069.87002 & -8069.07103 & -8068.98316 & -8069.22631 & -8069.21349 \\
\hline INT4 & -8069.91346 & -8069.11250 & -8069.02502 & -8069.26777 & -8069.25478 \\
\hline TS5 & -8069.89017 & -8069.09190 & -8069.00506 & -8069.24520 & -8069.23205 \\
\hline INT5 & -8069.90370 & -8069.10077 & -8069.01235 & -8069.26547 & -8069.25293 \\
\hline 3a...CsF & -857.89434 & -857.50784 & -857.47352 & -857.58978 & -857.57726 \\
\hline 3a & -737.77071 & -737.38539 & -737.35669 & -737.45382 & -737.44140 \\
\hline 2a-s1 & -306.51908 & -306.42388 & -306.41404 & -306.46147 & -306.44870 \\
\hline 2a-s2 & -170.85421 & -170.80366 & -170.79716 & -170.83541 & -170.82582 \\
\hline 2a-s3 & -78.59722 & -78.54638 & -78.54144 & -78.57349 & -78.56513 \\
\hline 2a-s4 & -117.92010 & -117.84057 & -117.83410 & -117.87127 & -117.86163 \\
\hline INT2-s1 \\
(INT2-s2)
\end{tabular}

Table S2-3. The electronic energies (Eele/a.u.), total energies ( $E_{\text {tot }} /$ a.u. $=E_{\text {ele }} /$ a.u. $\left.+Z P E / a . u.\right)$, enthalpy (H/a.u.), Gibbs free energies in gas $\left(\mathrm{G}_{1} / \mathrm{a} . u\right.$. $)$ and Gibbs free energies in solution $\left(\mathrm{G}_{2} /\right.$ a.u. $)$ for the alternative pathways, computed at IDSCRF(PCM)-B3LYP/DZVP level in DMA at 353K.

\begin{tabular}{||l|l|l|l|l|l||}
\hline \hline Species & $\mathrm{E}_{\text {ele }} /$ a.u. & $\mathrm{E}_{\text {tot }} /$ a.u. & H/a.u. & $\mathrm{G}_{1} /$ a.u. & $\mathrm{G}_{2} /$ a.u. \\
\hline TS2i & -7525.25651 & -7524.64407 & -7524.57639 & -7524.77088 & -7524.75795 \\
\hline INT2i & -7525.26591 & -7524.65171 & -7524.58372 & -7524.77952 & -7524.76667 \\
\hline COM1n & -7636.51428 & -7635.91524 & -7635.84737 & -7636.04034 & -7636.02717 \\
\hline TS1n & -7949.71146 & -7948.91259 & -7948.83037 & -7949.05624 & -7949.04376 \\
\hline INT1n & -7949.79964 & -7948.99671 & -7948.91516 & -7949.13912 & -7949.12643 \\
\hline TS2n & -7949.74639 & -7948.94542 & -7948.86403 & -7949.08679 & -7949.07417 \\
\hline INT2n & -7949.79666 & -7948.99281 & -7948.91137 & -7949.13470 & -7949.12184 \\
\hline
\end{tabular}

Table S2-4. The electronic energies (Eele/a.u.), total energies ( $E_{\text {tot }} /$ a.u. $\left.=E_{\text {ele }} / a . u .+Z P E / a . u.\right)$, enthalpy (H/a.u.), Gibbs free energies in gas $\left(\mathrm{G}_{1} /\right.$ a.u. $)$ and Gibbs free energies in solution $\left(\mathrm{G}_{2} /\right.$ a.u. $)$ for the rate determining step when phosphine ligand is $\mathrm{P}\left(4-\mathrm{F}_{3} \mathrm{CC}_{6} \mathrm{H}_{4}\right)_{3}, \mathrm{PPh}_{2}\left(\mathrm{C}_{6} \mathrm{~F}_{5}\right)$ or $\mathrm{P}(\mathrm{OMe})_{3}$, 
computed at IDSCRF(PCM)-B3LYP/DZVP level in DMA at 353K.

\begin{tabular}{|c|c|c|c|c|c|}
\hline Species & Eele/a.u. & $\mathrm{E}_{\text {tot }} / \mathrm{a} . \mathrm{u}$. & H/a.u. & $\mathrm{G}_{1}$ /a.u. & $\mathrm{G}_{2} /$ a.u. \\
\hline INT2b & -6634.06437 & -6633.46088 & -6633.40223 & -6633.57202 & -6633.55919 \\
\hline TS3b & -7058.51016 & -7057.72488 & -7057.65116 & -7057.85652 & -7057.84353 \\
\hline INT3b & -7058.51474 & -7057.72803 & -7057.65400 & -7057.86099 & -7057.84812 \\
\hline TS4b & -7058.50848 & -7057.72187 & -7057.64884 & -7057.85153 & -7057.83892 \\
\hline INT4b & -7058.55020 & -7057.76186 & -7057.68921 & -7057.89047 & -7057.87769 \\
\hline INT2c & -7130.33089 & -7129.76801 & -7129.70356 & -7129.88657 & -7129.87328 \\
\hline TS3c & -7554.77487 & -7554.03029 & -7553.95074 & -7554.16859 & -7554.15571 \\
\hline INT3c & -7554.77826 & -7554.03238 & -7553.95249 & -7554.17140 & -7554.15852 \\
\hline TS4c & -7554.76983 & -7554.02413 & -7553.94527 & -7554.16058 & -7554.14761 \\
\hline INT4c & -7554.81746 & -7554.06950 & -7553.99119 & -7554.20292 & -7554.19006 \\
\hline INT2d & -6284.57526 & -6284.11495 & -6284.06538 & -6284.21512 & -6284.20295 \\
\hline TS3d & -6709.01825 & -6708.37636 & -6708.31157 & -6708.49917 & -6708.48703 \\
\hline INT3d & -6709.02060 & -6708.37741 & -6708.31232 & -6708.49990 & -6708.48770 \\
\hline TS4d & -6709.00934 & -6708.36649 & -6708.30227 & -6708.48762 & -6708.47562 \\
\hline INT4d & -6709.06871 & -6708.42324 & -6708.35992 & -6708.53984 & -6708.52773 \\
\hline TS1b & -6634.03594 & -6633.43234 & -6633.37424 & -6633.54259 & -6633.52990 \\
\hline INT1b & -6634.06496 & -6633.45994 & -6633.40155 & -6633.56861 & -6633.55573 \\
\hline TS2b & -6634.04379 & -6633.44364 & -6633.38547 & -6633.55476 & -6633.54177 \\
\hline TS5b & -7058.52784 & -7057.74238 & -7057.67022 & -7057.86902 & -7057.85602 \\
\hline INT5b & -7058.54277 & -7057.75213 & -7057.67858 & -7057.88812 & -7057.87568 \\
\hline TS1c & -7130.30245 & -7129.73961 & -7129.67578 & -7129.85665 & -7129.84364 \\
\hline INT1c & -7130.32994 & -7129.76581 & -7129.70163 & -7129.88163 & -7129.86854 \\
\hline TS2c & -7130.31092 & -7129.75082 & -7129.68715 & -7129.86709 & -7129.85383 \\
\hline TS5c & -7554.79453 & -7554.04949 & -7553.97172 & -7554.18173 & -7554.16862 \\
\hline INT5c & -7554.81859 & -7554.06817 & -7553.98926 & -7554.20821 & -7554.19560 \\
\hline TS1d & -6284.54549 & -6284.08500 & -6284.03623 & -6284.18291 & -6284.17059 \\
\hline INT1d & -6284.57164 & -6284.10993 & -6284.06088 & -6284.20709 & -6284.19475 \\
\hline TS2d & -6284.55516 & -6284.09754 & -6284.04886 & -6284.19635 & -6284.18401 \\
\hline TS5d & -6709.05021 & -6708.40766 & -6708.34485 & -6708.52321 & -6708.51123 \\
\hline INT5d & -6709.09440 & -6708.44641 & -6708.38258 & -6708.56847 & -6708.55653 \\
\hline CATb & -6320.81321 & -6320.40814 & -6320.36536 & -6320.49686 & -6320.48286 \\
\hline CATc & -6817.08144 & -6816.71712 & -6816.66862 & -6816.81275 & -6816.79818 \\
\hline CATd & -5971.32189 & -5971.06005 & -5971.02652 & -5971.13538 & -5971.12215 \\
\hline $\mathbf{P P h}_{3}$ & -1036.32703 & -1036.05462 & -1036.03139 & -1036.11356 & -1036.09965 \\
\hline $\mathrm{PPh}_{2}\left(\mathrm{C}_{6} \mathrm{~F}_{5}\right)$ & -1532.59435 & -1532.36276 & -1532.33370 & -1532.42948 & -1532.41498 \\
\hline $\mathrm{P}(\mathrm{OMe})_{3}$ & -686.82366 & -686.69529 & -686.68073 & -686.74133 & -686.72946 \\
\hline
\end{tabular}

Table S2-5. The electronic energies (Eele/a.u.), total energies ( $E_{\text {tot }} /$ a.u. $=E_{\text {ele }} /$ a.u. $\left.+Z P E / a . u.\right)$, enthalpy (H/a.u.), Gibbs free energies in gas $\left(\mathrm{G}_{1} /\right.$ a.u. $)$ and Gibbs free energies in solution $\left(\mathrm{G}_{2} /\right.$ a.u. $)$ for the ligand exchange steps with the introduction of $\mathrm{CsCl}, \mathrm{LiF}, \mathrm{NaF}$ or $\mathrm{KF}$, computed at 
IDSCRF(PCM)-B3LYP/DZVP level in DMA at 353K.

\begin{tabular}{|c|c|c|c|c|c|}
\hline Species & Eele/a.u. & $\mathrm{E}_{\text {tot }} / \mathrm{a} . \mathrm{u}$ & H/a.u. & $\mathrm{G}_{1} / \mathrm{a} . \mathrm{u}$. & $\mathrm{G}_{2} /$ a.u. \\
\hline $\mathrm{CsCl}$ & -480.46291 & -480.46270 & -480.45787 & -480.49442 & -480.48452 \\
\hline LE-TS1Ba & -5956.83056 & -5956.51840 & -5956.48835 & -5956.58723 & -5956.57280 \\
\hline LE-INT1Ba & -5956.86060 & -5956.54792 & -5956.51710 & -5956.61917 & -5956.60523 \\
\hline LE-TS2Ba & -8004.53937 & -8003.94140 & -8003.87203 & -8004.07064 & -8004.05673 \\
\hline LE-INT2Ba & -8004.54107 & -8003.94267 & -8003.87258 & -8004.07030 & -8004.05621 \\
\hline LE-TS3Ba & -8004.53295 & -8003.93548 & -8003.86559 & -8004.06341 & -8004.04939 \\
\hline CATBa & -7692.49808 & -7692.08100 & -7692.02309 & -7692.19667 & -7692.18283 \\
\hline LiF & -107.47464 & -107.47287 & -107.46880 & -107.49667 & -107.48976 \\
\hline LE-TS1Bb & -5583.87235 & -5583.55706 & -5583.52875 & -5583.61833 & -5583.60478 \\
\hline LE-INT1Bb & -5583.89669 & -5583.58111 & -5583.55205 & -5583.64420 & -5583.63045 \\
\hline LE-TS2Bb & -7631.58033 & -7630.97939 & -7630.91169 & -7631.10427 & -7631.09100 \\
\hline LE-INT2Bb & -7631.58313 & -7630.98158 & -7630.91330 & -7631.10359 & -7631.09021 \\
\hline LE-TS3Bb & -7631.57285 & -7630.97191 & -7630.90391 & -7631.09425 & -7631.08082 \\
\hline CATBb & -7319.53332 & -7319.11312 & -7319.05702 & -7319.22150 & -7319.20811 \\
\hline TS1Bb & -7632.76676 & -7632.14761 & -7632.07589 & -7632.27905 & -7632.26612 \\
\hline INT1Bb & -7632.80449 & -7632.18471 & -7632.11241 & -7632.31745 & -7632.30422 \\
\hline INT2Bb & -7632.77587 & -7632.15709 & -7632.08482 & -7632.29148 & -7632.27843 \\
\hline INT1Bb...DMA & -7920.69615 & -7919.94427 & -7919.85974 & -7920.09473 & -7920.08238 \\
\hline INT2Bb...DMA & -7920.68007 & -7919 & -7919.84509 & -7920.08267 & -7920.07028 \\
\hline LiF...DMA & -395.38380 & -395.24975 & -395.23376 & -395.30044 & -395.28848 \\
\hline $\mathrm{NaF}$ & -262.24058 & -262.23962 & -262.23529 & -262.26561 & -262.25840 \\
\hline LE-TS1Bc & -5738.62814 & -5738.31457 & -5738.28555 & -5738.37730 & -5738.36311 \\
\hline LE-INT1Bc & -5738.65817 & -5738.34406 & -5738.31429 & -5738.40877 & -5738.39492 \\
\hline LE-TS2Bc & - & - & - & - & - \\
\hline LE-INT2Bc & -7786.34271 & -7785.74260 & -7785.67368 & -7785.86526 & -7785.85176 \\
\hline LE-TS3Bc & -7786.33233 & -7785.73309 & -7785.66427 & -7785.85949 & -7785.84571 \\
\hline CATBc & -7474.29474 & -7473.87609 & -7473.81925 & -7473.98653 & -7473.97297 \\
\hline TS1Bc & -7787.52132 & -7786.90416 & -7786.83167 & -7787.03860 & -7787.02575 \\
\hline INT1Bc & -7787.54937 & -7786.93100 & -7786.85826 & -7787.06378 & -7787.05063 \\
\hline INT2Bc & -7787.53837 & -7786.92072 & -7786.84782 & -7787.05440 & -7787.04115 \\
\hline $\mathbf{K F}$ & -699.83436 & -699.83373 & -699.82922 & -699.86114 & -699.85326 \\
\hline LE-TS1Bd & -6176.21705 & -6175.90421 & -6175.87483 & -6175.96869 & -6175.95419 \\
\hline LE-INT1Bd & -6176.25067 & -6175.93720 & -6175.90706 & -6176.00391 & -6175.98937 \\
\hline LE-TS2Bd & -8223.93263 & -8223.33402 & -8223.26523 & -8223.46143 & -8223.44750 \\
\hline LE-INT2Bd & -8223.93435 & -8223.33495 & -8223.26568 & -8223.45863 & -8223.44500 \\
\hline LE-TS3Bd & -8223.92390 & -8223.32539 & -8223.25617 & -8223.45123 & -8223.43789 \\
\hline CATBd & -7911.88719 & -7911.46924 & -7911.41198 & -7911.58084 & -7911.56648 \\
\hline TS1Bd & -8225.11017 & -8224.49383 & -8224.42104 & -8224.62931 & -8224.61634 \\
\hline INT1Bd & -8225.13744 & -8224.51987 & -8224.44673 & -8224.65553 & -8224.64208 \\
\hline INT2Bd & -8225.13306 & -8224.51593 & -8224.44278 & -8224.65079 & -8224.63755 \\
\hline
\end{tabular}




\begin{tabular}{|l|l|l|l|l|l||}
\hline \hline TS2Bd & -8225.11832 & -8224.50580 & -8224.43306 & -8224.64129 & -8224.62808 \\
\hline TS2Bc & -7787.52634 & -7786.91291 & -7786.84070 & -7787.04701 & -7787.03380 \\
\hline TS2Bb & -7632.76720 & -7632.15295 & -7632.08133 & -7632.28624 & -7632.27318 \\
\hline \hline
\end{tabular}

Table S2-6. The electronic energies (Eele/a.u.), total energies ( $E_{\text {tot }} /$ a.u. $\left.=E_{\text {ele }} / a . u .+Z P E / a . u.\right)$, enthalpy (H/a.u.), Gibbs free energies in gas $\left(\mathrm{G}_{1} /\right.$ a.u. $)$ and Gibbs free energies in solution $\left(\mathrm{G}_{2} /\right.$ a.u.) for the entire catalytic cycle when $\mathrm{Q}=2$-methyl-8-quinolinolato, computed at IDSCRF(PCM)-B3LYP/DZVP level in DMA at 353K. (simplified model: $\mathrm{PPh}_{3}$ as ligand)

\begin{tabular}{|l|l|l|l|l|l||}
\hline Species & $\mathrm{E}_{\text {ele }} /$ a.u. & $\mathrm{E}_{\text {tot }}$ a.u. & H/a.u. & $\mathrm{G}_{1} /$ a.u. & $\mathrm{G}_{2} /$ a.u. \\
\hline preCATm & -5515.72551 & -5515.38560 & -5515.35874 & -5515.44494 & -5515.43147 \\
\hline LE-TS1m & -5635.81555 & -5635.47574 & -5635.44370 & -5635.54637 & -5635.53197 \\
\hline LE-INT1m & -5635.84819 & -5635.50731 & -5635.47468 & -5635.57929 & -5635.56495 \\
\hline LE-TS2m & -6672.16667 & -6671.55319 & -6671.49672 & -6671.65781 & -6671.64452 \\
\hline LE-INT2m & -6672.17286 & -6671.55882 & -6671.50182 & -6671.65995 & -6671.64621 \\
\hline LE-TS3m & -6672.16172 & -6671.54882 & -6671.49174 & -6671.65319 & -6671.63940 \\
\hline CATm & -6360.12223 & -6359.68943 & -6359.64461 & -6359.77803 & -6359.76414 \\
\hline TS1m & -6673.34481 & -6672.71315 & -6672.65289 & -6672.82532 & -6672.81206 \\
\hline INT1m & -6673.37475 & -6672.74219 & -6672.68152 & -6672.85343 & -6672.84063 \\
\hline TS2m & -6673.35288 & -6672.72529 & -6672.66486 & -6672.83811 & -6672.82482 \\
\hline INT2m & -6673.37296 & -6672.74194 & -6672.68104 & -6672.85392 & -6672.84083 \\
\hline TS3m & -7097.82001 & -7097.00741 & -7096.93121 & -7097.14308 & -7097.13051 \\
\hline INT3m & -7097.82504 & -7097.01089 & -7096.93449 & -7097.14613 & -7097.13342 \\
\hline TS4m & -7097.82029 & -7097.00626 & -7096.93087 & -7097.13919 & -7097.12625 \\
\hline INT4m & -7097.85945 & -7097.04314 & -7096.96847 & -7097.17104 & -7097.15827 \\
\hline TS5m & -7097.83619 & -7097.02284 & -7096.94868 & -7097.14967 & -7097.13717 \\
\hline INT5m & -7097.86430 & -7097.04592 & -7096.97041 & -7097.18107 & -7097.16878 \\
\hline \hline
\end{tabular}

Table S2-7. The electronic energies (Eele/a.u.) for the fragments of INT2/INT2b/INT2c/INT2d and TS4/TS4b/TS4c/TS4d, computed at IDSCRF(PCM)-B3LYP/DZVP level in DMA at 353K.

\begin{tabular}{|l|l|l|l||}
\hline Species & E ele/a.u. & Species & E $_{\text {ele }} /$.u. \\
\hline INT2b-Fragment I & -1036.32508 & INT2b-Fragment II & -5597.68088 \\
\hline TS4b-Fragment I & -1036.32444 & TS4b-Fragment II & -6022.12366 \\
\hline INT2-Fragment I & -2047.68593 & INT2-Fragment II & -5597.67999 \\
\hline TS4-Fragment I & -2047.68687 & TS4-Fragment II & -6022.12694 \\
\hline INT2c-Fragment I & -1532.59113 & INT2c-Fragment II & -5597.68127 \\
\hline TS4c-Fragment I & -1532.59183 & TS4c-Fragment II & -6022.12346 \\
\hline INT2d-Fragment I & -686.82300 & INT2d-Fragment II & -5597.68204 \\
\hline TS4d-Fragment I & -686.81483 & TS4d-Fragment II & -6022.12548 \\
\hline
\end{tabular}

Table S2-8. The electronic energies ( $E_{\text {ele }} /$ a.u.) for CAT, INT1, INT2, INT3, INT4 and INT5 with different spin multiplicities. 


\begin{tabular}{|l|l|l|l||}
\hline \hline Singlet State & Eele/a.u. & Triplet State & E ele/a.u. \\
\hline CAT & -7332.17606 & CAT-03 & -7332.11092 \\
\hline INT1 & -7645.42688 & INT1-03 & -7645.35466 \\
\hline INT2 & -7645.42406 & INT2-03 & -7645.35177 \\
\hline INT3 & -8069.87361 & INT3-03 & -8069.83037 \\
\hline INT4 & -8069.91346 & INT4-03 & -8069.83312 \\
\hline INT5 & -8069.90370 & INT5-03 & -8069.88031 \\
\hline \hline
\end{tabular}




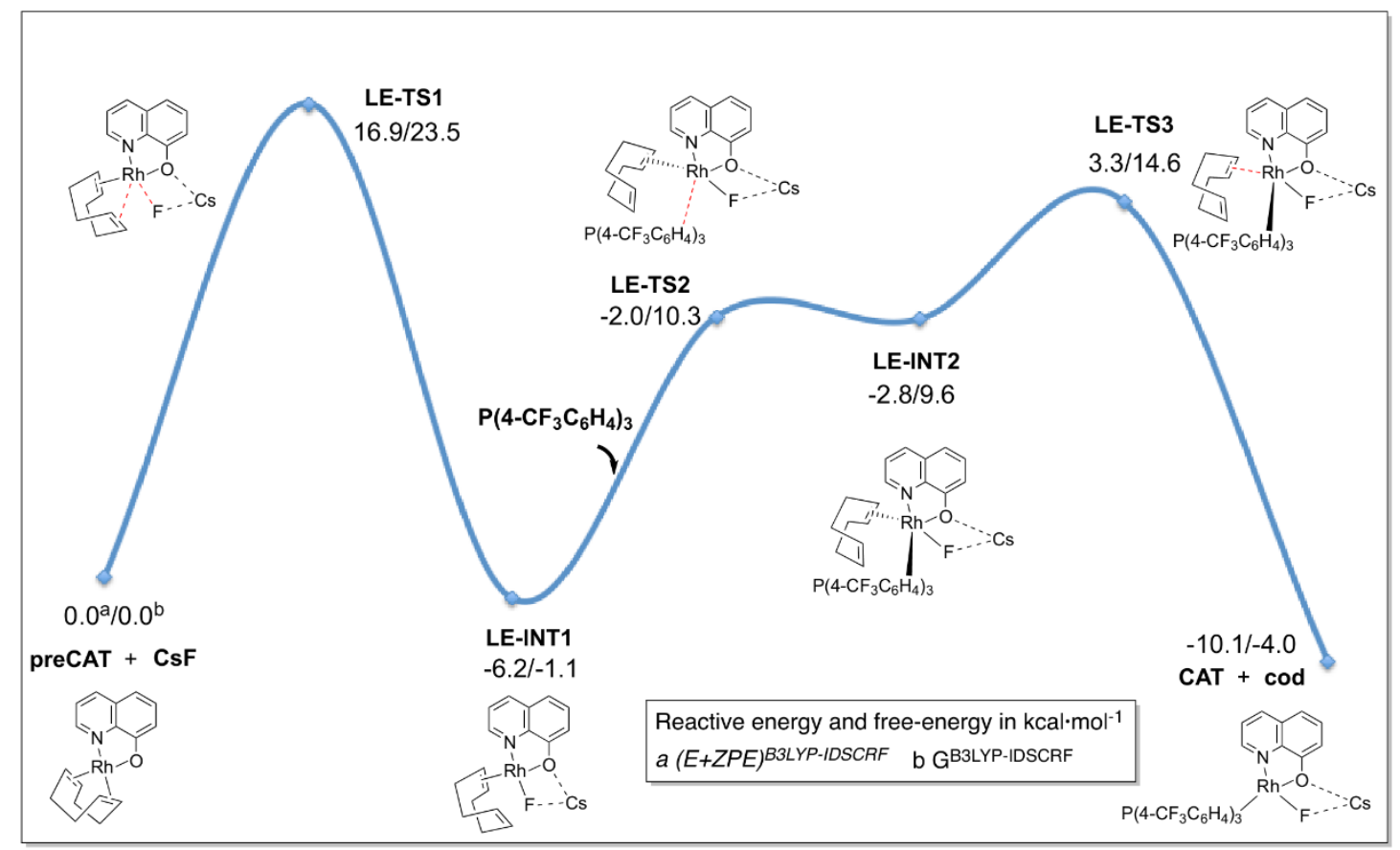

Figure S1. Gibbs free energy profiles $\left(\mathrm{kcal} \cdot \mathrm{mol}^{-1}\right)$ for the optimal ligand exchange process, computed at B3LYP+IDSCRF/DZVP level in acetonitrile at 353K. 


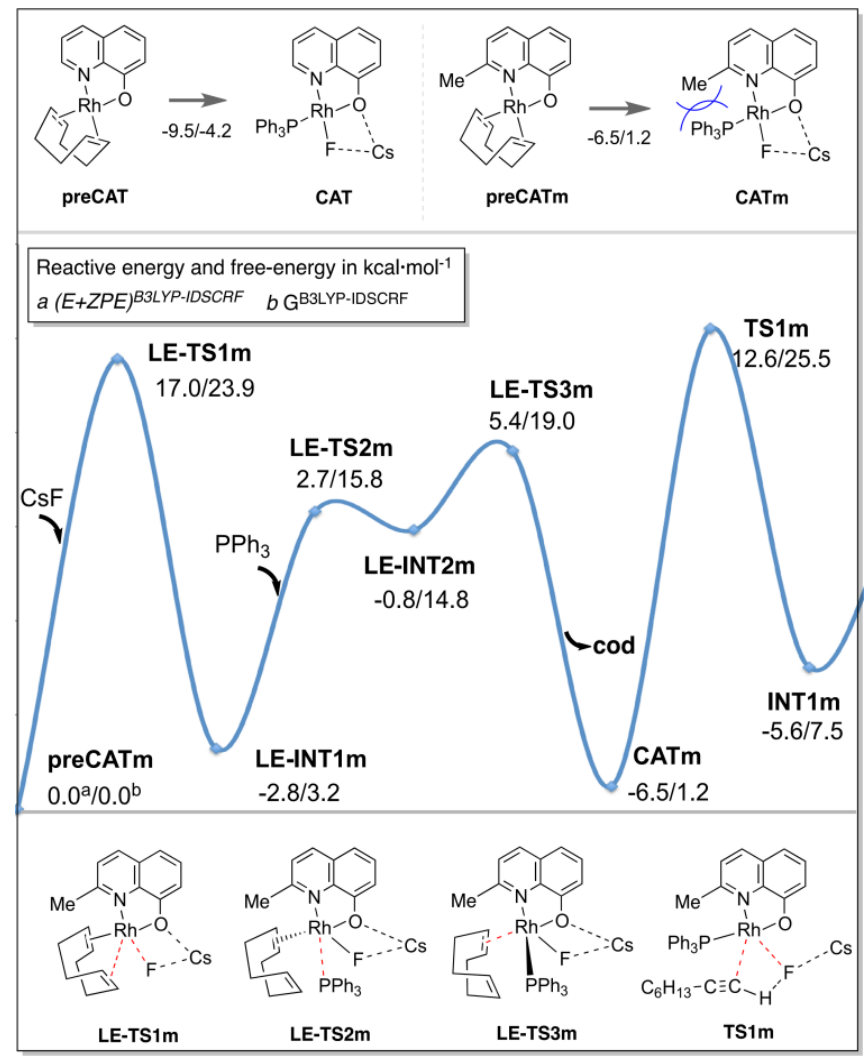

Figure S2. Gibbs free energy profiles for the ligand and substrate exchange process with the presence of 2-methyl-8-quinolinolato, along with geometrical structures of preCAT, preCATm, CAT, CATm and some transition states, computed at B3LYP+IDSCRF/DZVP level in acetonitrile at $353 \mathrm{~K}$. (simplified model: $\mathrm{PPh}_{3}$ as ligand) 




Figure S3. Gibbs free energy profiles $\left(\mathrm{kcal} \cdot \mathrm{mol}^{-1}\right)$ for the entire catalytic cycle when $\mathrm{Q}=2$-methyl-8-quinolinolato, computed at B3LYP+IDSCRF/DZVP level in acetonitrile at 353K. (simplified model: $\mathrm{PPh}_{3}$ as ligand) 


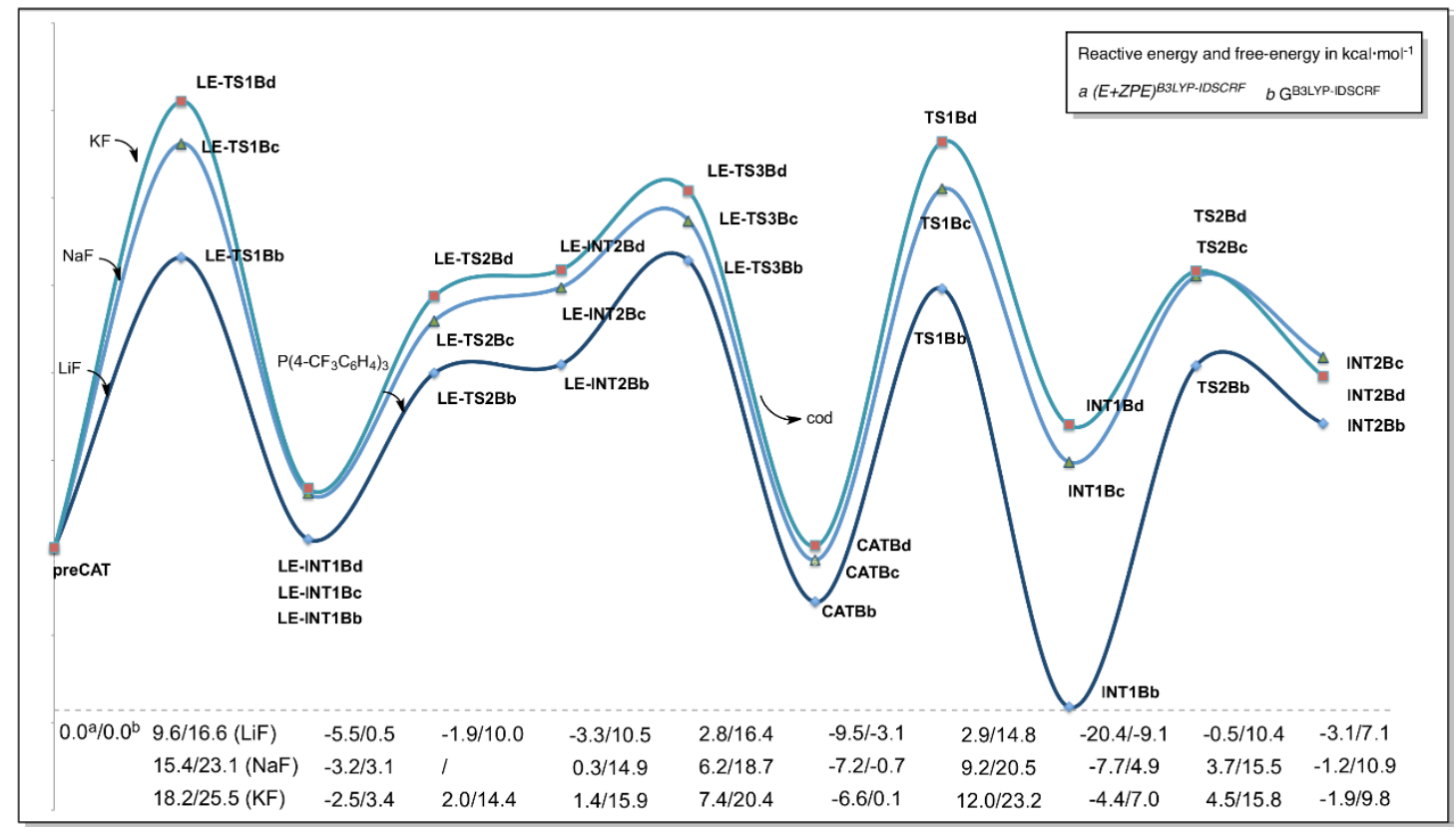

Figure S4. Gibbs free energy profiles $\left(\mathrm{kcal} \cdot \mathrm{mol}^{-1}\right)$ for the ligand exchange and subsequent steps when $\mathrm{KF}, \mathrm{NaF}$ and $\mathrm{LiF}$ as base, computed at B3LYP+IDSCRF/DZVP level in acetonitrile at $353 \mathrm{~K}$. 


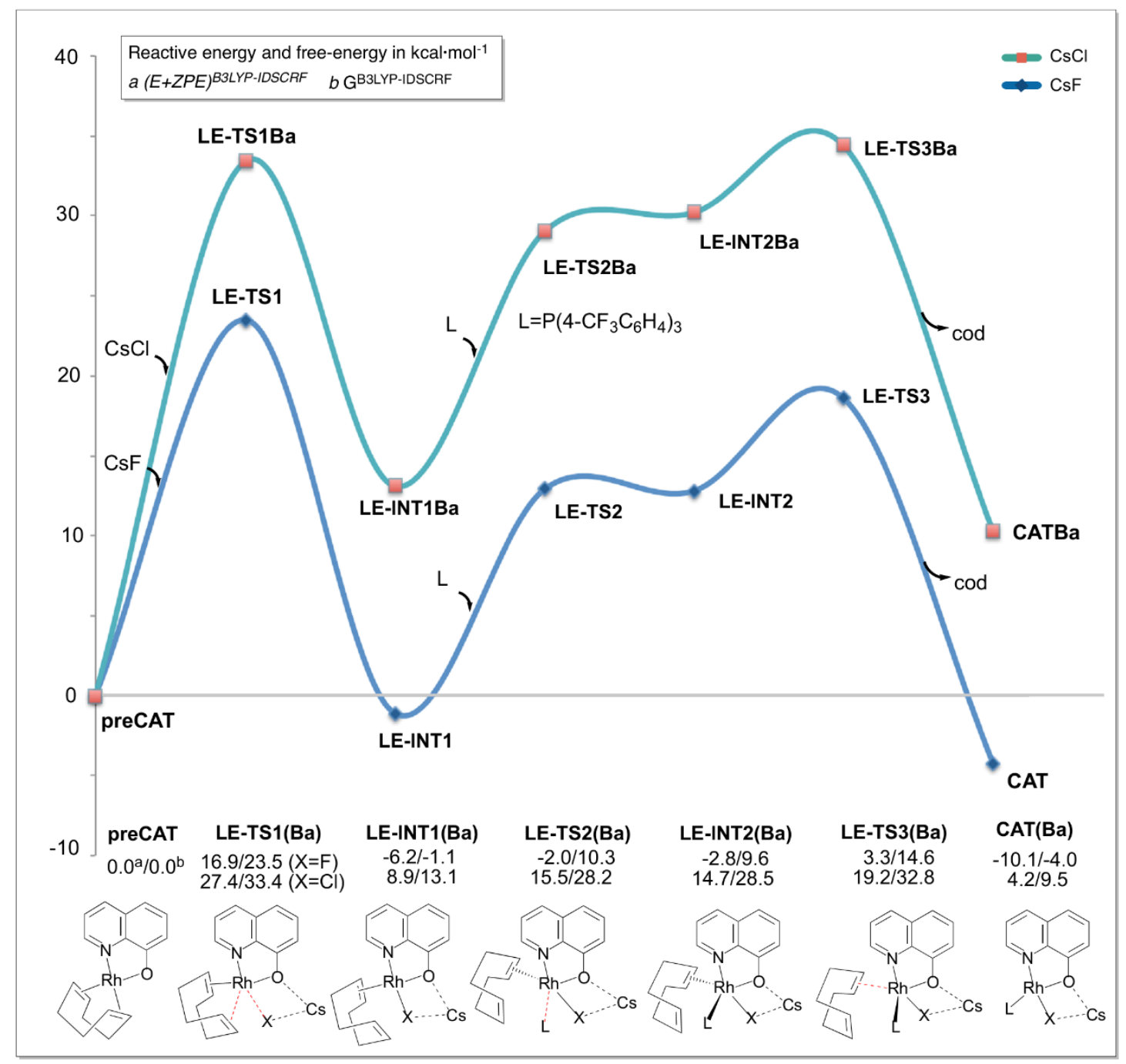

Figure S5. Gibbs free energy profiles $\left(\mathrm{kcal} \cdot \mathrm{mol}^{-1}\right)$ for the ligand exchange process with the introduction of $\mathrm{CsF}$ and $\mathrm{CsCl}$, along with geometrical structures of stationary points along the reaction pathway, computed at B3LYP+IDSCRF/DZVP level in acetonitrile at 353K. 